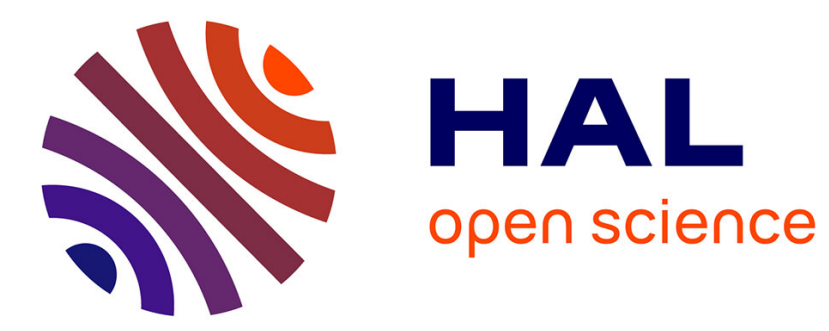

\title{
SYMMETRIC POWER CONGRUENCE IDEALS AND SELMER GROUPS
}

Haruzo Hida, Jacques Tilouine

\section{To cite this version:}

Haruzo Hida, Jacques Tilouine. SYMMETRIC POWER CONGRUENCE IDEALS AND SELMER GROUPS. 2016. hal-01398905

\section{HAL Id: hal-01398905 \\ https://hal.science/hal-01398905}

Preprint submitted on 18 Nov 2016

HAL is a multi-disciplinary open access archive for the deposit and dissemination of scientific research documents, whether they are published or not. The documents may come from teaching and research institutions in France or abroad, or from public or private research centers.
L'archive ouverte pluridisciplinaire HAL, est destinée au dépôt et à la diffusion de documents scientifiques de niveau recherche, publiés ou non, émanant des établissements d'enseignement et de recherche français ou étrangers, des laboratoires publics ou privés. 


\title{
SYMMETRIC POWER CONGRUENCE IDEALS AND SELMER GROUPS
}

\author{
HARUZO HIDA AND JACQUES TILOUINE
}

\section{Contents}

1. Introduction 1

2. Theorems $R_{n-1}=\mathbf{T}_{n-1}^{u}$ for $n \geq 4$

2.1. Big ordinary Hecke algebra for unitary groups $\quad 4$

2.2. Symm ${ }^{n-1}$ Langlands functoriality 6

2.3. Galois representations $r$

2.4. Galois cohomology 11

2.5. Application of Chebotarev density theorem 13

2.6. Construction of a Taylor-Wiles system 14

2.7. End of the proof 16

3. Proof of Theorem $1.3 \quad 17$

3.1. The case $j=3 \quad 17$

3.2. The case $j=2 \quad 19$

4. The case $j=4 \quad 21$

5. Digression: a Kummer type criterion for the non triviality of certain Selmer groups 22

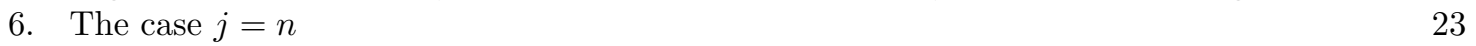

7. The case of the standard representation of GSp(4) 24

8. Congruence ideal formalism 24

8.1. Differentials 24

8.2. Congruence and differential modules 26

8.3. Transfer property of congruence modules 26

8.4. Local complete intersections 28

8.5. Proof of Tate's theorem 29

8.6. A more general setting 32

$\begin{array}{ll}\text { References } & 33\end{array}$

\section{INTRODUCTION}

In this paper we define relative congruence ideals for various automorphic symmetric powers Symm $^{m} \mathbf{f}$ of a Hida family $\mathbf{f}$ over $\mathbb{Q}$ in big ordinary Hecke algebras for symplectic and unitary groups (these powers are now known to be automorphic for $m \leq 8$ ) and we prove, under some assumptions, that they coincide with the characteristic power series of (the Pontryagin duals of) Greenberg Selmer groups over $\mathbb{Q}$ for related symmetric powers $\mathcal{A}_{\mathbf{f}}^{n}=\operatorname{Symm}^{2 n} \otimes \operatorname{det}^{-n} \rho_{\mathbf{f}}$ of the Galois representation of the family $\mathbf{f}$. Note that these Selmer groups over $\mathbb{Q}$ are modules over the weight variable Iwasawa algebra which are finitely generated but a priori not known to be torsion except for the symmetric square. It follows from our result that they are. Similar results when one includes the cyclotomic variable (that is, for Selmer groups over the $\mathbb{Z}_{p}$-extension $\mathbb{Q}_{\infty}$ of $\mathbb{Q}$ ) could probably also be studied but are not dealt with in this paper. Let us be a little more precise.

Let $N \geq 1$ and let $p$ be an odd prime not dividing $N$. Let $\Lambda_{1}=\mathbb{Z}_{p}[[X]]$ be the one variable Iwasawa algebra identified to the completed group algebra of $1+p \mathbb{Z}_{p}$ by the choice of a topological generator

Date: November 10, 2016.

The first author is partially supported by the NSF grant: DMS 1464106. The second author is partially supported by the ANR grant: PerCoLaTor ANR-14-CE25. 
$u$ of the group $1+p \mathbb{Z}_{p}$. Let $h_{1}$ be the cuspidal Hida Hecke algebra (generated by the $T_{\ell}$ 's for $\ell$ prime to $N p$, by the diamond operators $\left\langle a>_{0}\right.$ for $a \in \mathbb{Z}_{p}^{\times}$for the cohomological weight 0 , and by $U_{p}$. The ring $h_{1}$ is reduced; it is endowed with a structure of $\Lambda_{1}$-algebra by the homomorphism sending $X$ to $\langle u\rangle_{0}-1$ for which it is finite and flat. Let $\mu: h_{1} \rightarrow A_{1}$ be a surjective $\Lambda_{1}$-algebra homomorphism onto a local domain which is finite and torsion free over $\Lambda_{1}$. Geometrically, it amounts to considering an irreducible component of $\operatorname{Spec} h_{1}$. Let $k=A_{1} / \mathfrak{m}_{A_{1}}$ be the residue field of $A_{1}$. Let $\Gamma_{\mathbb{Q}}$ be the absolute Galois group. We assume that the residual Galois representation $\bar{\rho}_{\mu}: \Gamma_{\mathbb{Q}} \rightarrow \mathrm{GL}_{2}(k)$ associated to $\mu$ is irreducible. It is well-known that under this assumption there exists a continuous Galois representation $\rho_{\mu}: \Gamma_{\mathbb{Q}} \rightarrow \mathrm{GL}_{2}\left(A_{1}\right)$ associated to $\mu$. Let $\omega$ be the cyclotomic character modulo $p$ (we shall also denote by $\omega$ its Teichmüller lift). The ordinarity condition for $\mu$ implies that there exists a unique integer $a \in[0, p-2]$ such that the restriction of the representation $\bar{\rho}_{\mu}$ to an inertia group $I_{p}$ at $p$ is conjugate to

$$
\left(\begin{array}{cc}
1 & * \\
0 & \omega^{-a-1}
\end{array}\right)
$$

The integer $a$ is fixed throughout the paper. Let $\mathrm{St}_{2}$ be the standard representation of $\mathrm{GL}_{2}$. For any $j \geq 1$, let $\mathcal{A}^{j}=\mathrm{Symm}^{2 j} \otimes \operatorname{det}^{-j} \mathrm{St}_{2}$; we assume from now on that $p>2 j+1$; in particular, viewed as a $\mathbb{Z}_{p^{-}}$schematic representation, the $(2 j+1)$-dimensional representation of $\mathrm{GL}_{2}, \mathcal{A}^{j}$ has irreducible geometric fibers. Let $\mathcal{A}_{\mu}^{j}=\mathcal{A}^{j} \circ \rho_{\mu}: \Gamma_{\mathbb{Q}} \rightarrow \mathrm{GL}_{2 j+1}\left(A_{1}\right)$. Its restriction to the local Galois group $\Gamma_{\mathbb{Q}_{p}}$ leaves stable a decreasing filtration $\left(F^{k} \mathcal{A}_{\mu}^{j}\right)_{k}$ with $A_{1}$-free graded pieces gr ${ }^{k} \mathcal{A}_{\mu}^{j}$ on which the inertia subgroup $I_{p}$ acts by $\mathbb{X}^{k}$. Here, $\mathbb{X}: \Gamma_{\mathbb{Q}} \rightarrow \Lambda_{1}^{\times}$denotes the restriction (to $I_{p}$ ) of the universal deformation of the $p$-adic cyclotomic character $\chi$ unramified outside $p \infty$ : if $\chi(\sigma)=\omega(\sigma) u^{\ell(\sigma)}($ that is, $\ell\left(\left[z, \mathbb{Q}_{p}\right]\right)=\log _{p}(z) / \log _{p}(u)$ for $\left.z \in \mathbb{Z}_{p}^{\times}\right)$. then

$$
\mathbb{X}(\sigma)=\chi(\sigma)(1+X)^{\ell(\sigma)}
$$

Let $\widetilde{A}_{1}$ be the normal closure of $A_{1}$. It is a two dimensional normal local ring, hence it is CohenMacaulay so that $\widetilde{A}_{1}$ is free over $\Lambda_{1}$. For any $\mathbb{Z}_{p}$-module $M$, we denote by $M^{*}=\operatorname{Hom}\left(M, \mathbb{Q}_{p} / \mathbb{Z}_{p}\right)$ its Pontryagin dual. Let us consider the minimal $p$-ordinary Selmer group associated to $\mathcal{A}_{\mu}^{j}$ :

$$
\operatorname{Sel}\left(\mathcal{A}_{\mu}^{j}\right)=\operatorname{Ker}\left(\mathrm{H}^{1}\left(\mathbb{Q}, \mathcal{A}_{\mu}^{j} \otimes_{A_{1}} \widetilde{A}_{1}^{*}\right) \rightarrow \prod_{\ell \neq p} \mathrm{H}^{1}\left(I_{\ell}, \mathcal{A}_{\mu}^{j} \otimes_{A_{1}} \widetilde{A}_{1}^{*}\right) \times \mathrm{H}^{1}\left(I_{p},\left(\mathcal{A}_{\mu}^{j} / F^{1} \mathcal{A}_{\mu}^{j}\right) \otimes_{A_{1}} \widetilde{A}_{1}^{*}\right)\right) .
$$

Its Pontryagin dual $\operatorname{Sel}\left(\mathcal{A}_{\mu}^{j}\right)^{*}$ is finitely generated over $\widetilde{A}_{1}$. Recall that the Greenberg-Iwasawa main conjecture implies (by taking the cyclotomic variable $s$ to be 1 ) that

1) there is a $p$-adic $L$ function $L_{p}\left(\mathcal{A}_{\mu}^{j}\right)$ in $\widetilde{A}_{1}$ interpolating normalized special values $L^{*}\left(\mathcal{A}_{\mathbf{f}_{k}}^{j}, 1\right)$ where $\mathbf{f}_{k}$ runs over the eigenforms of classical weights $k \geq 2$ occuring in $\mu$.

2) $\operatorname{Sel}\left(\mathcal{A}_{\mu}^{j}\right)^{*}$ is torsion and a characteristic power series is equal to $L_{p}\left(\mathcal{A}_{\mu}^{j}\right)$ up to a unit in $\widetilde{A}_{1}$; this means, more precisely, that the localization at each height one prime of $\widetilde{A}_{1}$ of its first Fitting ideal $\operatorname{Fitt}_{0}\left(\operatorname{Sel}\left(\mathcal{A}_{\mu}^{j}\right)^{*}\right)$ is generated by $L_{p}\left(\mathcal{A}_{\mu}^{j}\right)$.

Let $\mathbf{T}_{1}$ be the localization of $h_{1}$ at the maximal ideal corresponding to the residual representation $\bar{\rho}_{\mu}$. The homomorphism $\mu$ factors through $\mathbf{T}_{1}$. We still denote $\mu: \mathbf{T}_{1} \rightarrow A_{1}$ the resulting homomorphism. Extending the scalars, it gives rise to a surjective homomorphism $\widetilde{\mathbf{T}}_{1}=\mathbf{T}_{1} \otimes_{\Lambda_{1}} \widetilde{A}_{1} \rightarrow \widetilde{A}_{1}$ which we again denote by $\mu$. The context should make clear the meaning of this notation. Since $\mathbf{T}_{1}$ is reduced and $\widetilde{A}_{1}$ is a domain which is flat over $\Lambda_{1}$, we see that $\widetilde{\mathbf{T}}_{1}$ is reduced too. By tensoring with $\mathcal{K}_{1}=\operatorname{Frac}\left(A_{1}\right)$, we have a splitting

$$
\widetilde{\mathbf{T}}_{1} \otimes_{\widetilde{A}_{1}} \mathcal{K}_{1} \cong \mathcal{K}_{1} \times \widetilde{\mathbf{T}}_{1, \mathcal{K}_{1}}^{\prime}
$$

where the first projection is given by $\mu \otimes \operatorname{Id}_{\mathcal{K}_{1}}$. Let $\widetilde{\mathbf{T}}_{1}^{\prime}$ be the image of the second projection $\widetilde{\mathbf{T}}_{1} \rightarrow \widetilde{\mathbf{T}}_{\mu, \mathcal{K}_{1}}^{\prime}$. One defines the congruence ideal of $\mu$ by $\mathfrak{c}_{\mu}=\widetilde{\mathbf{T}}_{1} \cap\left(\widetilde{A}_{1} \times\left\{0_{\widetilde{\mathbf{T}}_{\mu}^{\prime}}\right\}\right)$. We view this ideal as an ideal of $\widetilde{A}_{1}$. Recall that for a finitely generated $\widetilde{A}_{1}$-module $M$, any generator of the smallest principal ideal $\mathcal{X}_{M}$ containing the first Fitting ideal $\operatorname{Fitt}_{0}(M)$ of $M$ is called a characteristic power series : $\mathcal{X}_{M}=(\operatorname{Char}(M))$. It is non zero if and only if $M$ is torsion. Consider the assumption 
(*) $N$ is squarefree, there exists a subfield $k^{\prime} \subset k$ such that $\mathrm{SL}_{2}\left(k^{\prime}\right) \subset \operatorname{Im} \bar{\rho}_{\mu} \subset \mathrm{GL}_{2}\left(k^{\prime}\right)$, moreover for any prime $\ell$ dividing $N$, the restriction to $I_{\ell}$ of $\bar{\rho}_{\mu}$ is non trivial (hence unipotent).

Let $\alpha=\mu\left(U_{p}\right)$. Let $R_{1}$ be the universal deformation ring for $N$-minimal $p$-ordinary deformations of $\bar{\rho}_{\mu}$. Recall a special case of theorems by Wiles and Hida

Theorem 1.1. Assume $(*)$ and either $\alpha^{2} \not \equiv 1\left(\bmod \mathfrak{m}_{A_{1}}\right)$ or $a+1<p-1$, then $R_{1}=\mathbf{T}_{1}$ and $\mathbf{T}_{1}$ is local complete intersection over $\Lambda_{1}$. Moreover, $\mathfrak{c}_{\mu}$ is a principal ideal of $\widetilde{A}_{1}$.

The precise definition and some properties of local complete intersection algebras are given Section 8.4 below.

Corollary 1.2. Assume $(*)$ and $a+1<p-1$, then

(a) $\mathfrak{c}_{\mu}=\left(\operatorname{Char}\left(\operatorname{Sel}\left(\mathcal{A}_{\mu}^{1}\right)^{*}\right)\right)$,

(b) $\mathfrak{c}_{\mu}=\left(L_{p}\left(\mathcal{A}_{\mu}^{1}\right)\right)$.

Note that the $p$-adic $L$ function $L_{p}\left(\mathcal{A}_{\mu}^{1}\right)$ interpolating the special values $\left.L^{*}\left(\mathcal{A}_{\mathbf{f}_{k}}^{1}\right)\right)$ has been constructed by one of the authors [H88b] (see also his notes of the Pune course [H16]).

The goal of this paper is to establish analogues of part $(a)$ of the theorem above for higher $j$ 's, provided the automorphic base change is established for $\mathrm{Symm}^{m}$ for certain values of $m$ less than $2 j$.

Theorem 1.3. Assume $(*)$ and $3(a+1)<p-1$. Then,

- for $j=3$, the characteristic power series of $\operatorname{Sel}\left(\mathcal{A}_{\mu}^{j}\right)^{*}$ is a generator of the ideal of congruences between the family $\mathrm{Symm}^{3} \mu$ and Siegel families which are not of the form $\mathrm{Symm}^{3} \mu^{\prime}$ for other $\mathrm{GL}_{2}$-families $\mu^{\prime}$,

- for $j=2$, the characteristic power series of $\operatorname{Sel}\left(\mathcal{A}_{\mu}^{j}\right)^{*}$ is a generator of the congruence ideal between the family $\mathrm{Symm}^{3} \mu$ and families on U(4) which don't come from Siegel families.

Similarly, we have

Theorem 1.4. If one assumes besides $(*)$ that $4(a+1)<p-1$, then for $j=4$, the characteristic power series of $\operatorname{Sel}\left(\mathcal{A}_{\mu}^{j}\right)^{*}$ is a generator of the congruence ideal between the family $\operatorname{Symm}^{4} \mu$ and families of unitary forms on $U(5)$ which don't come from congruences between $\mathrm{Symm}^{3} \mu$ and families on $\mathrm{GSp}_{4}$ by the integral transfer from $\mathrm{GSp}_{4}$ to $U(5)$.

See Sections 3 and 4 for a more precise form of the statement and its proof. To put these results in perspective, let us mention a more elementary result.

Let $\mathfrak{p}$ be a prime of $\widetilde{A}_{1}$. For $j=3,2,4$, consider the condition

$$
\left(S_{j}\right) \quad \operatorname{Fitt}_{0}\left(\operatorname{Sel}\left(\mathcal{A}_{\mu}^{j}\right)^{*}\right) \subset \mathfrak{p}
$$

and the conditions

$\left(C_{3}\right)$ there exists a Hida family $G$ of Iwahori level $N$ on $\mathrm{GSp}_{4}$ which is not the Symm ${ }^{3}$ of a Hida family on $\mathrm{GL}_{2}$ and such that $\mathrm{Symm}^{3} \mu \equiv G(\bmod \mathfrak{p})$

$\left(C_{2}\right)$ there exists a Hida family $G$ of Iwahori level $N$ on $U(4)$ which does not come from $\mathrm{GSp}_{4}$ by base change and such that $\operatorname{Symm}^{3} \mu \equiv G(\bmod \mathfrak{p})$

$\left(C_{4}\right)$ there exists a Hida family $G$ of Iwahori level $N$ on $U(5)$ which does not come from GSp $\mathrm{GS}_{4}$ by base change and such that $\operatorname{Symm}^{4} \mu \equiv G(\bmod \mathfrak{p})$,

Theorem 1.5. Assume $(*)$, then $\left(C_{3}\right)$ implies $\left(S_{3}\right)$ or $\left(S_{2}\right)$ or $\left(S_{4}\right)$.

See Section 5. It requires a theorem of big image of Galois established by [HT15] when $A_{1}=\Lambda_{1}$ and by $\mathrm{A}$. Conti in his thesis [Con16b] in general. Note that the conditions $\left(C_{j}\right)$ are not mutually exclusive so that the difficulty of separating a priori the possible conclusions $\left(S_{j}\right)$ is not so surprising.

Our theorems do separate the conclusions and imply in particular for $j=3,2,4$ that $\left(C_{j}\right)$ implies $\left(S_{j}\right)$. Their proof require using more advanced tools, namely $R=T$ type theorems in the minimal level case and Hida-Tate theory of conguence ideals for Gorenstein rings. Actually our method applies to more cases: 
Theorem 1.6. Assume $(*)$ and that $p-1>n(a+1)$. Assume also that $N$ has at least two prime factors. Assume that the transfers $\mathrm{Symm}^{n-1}$ and $\mathrm{Symm}^{n}$ from $G L_{2}(\mathbb{Q})$ to $\mathrm{GL}_{n}$ resp. $G L_{n+1}$ are established.

Then, the characteristic power series of $\operatorname{Sel}\left(\mathcal{A}_{\mu}^{n}\right)^{*}$ is a generator of the quotient of the congruence ideal between the family $\mathrm{Symm}^{n} \mu$ and families of unitary forms on $U(n+1)$ by the congruence ideal between the family $\mathrm{Symm}^{n-1} \mu$ and families of unitary forms on $U(n)$. In particular, the quotient of these ideals is integral and principal.

This theorem applies for $n=5,6,7,8$ by [CT15] where the Symm ${ }^{m}$ transfer is established for $m \leq 8$. See Section 6 for a more precise statement and the proof.

We finally give an analogue result starting from a Hida family $\sigma$ on $\operatorname{GSp}_{4}(\mathbb{Q})$ instead of a Hida family $\mu$ on $\mathrm{GL}_{2}(\mathbb{Q})$. The method and result are similar although the Hida family $\sigma$ is two variable so that the commutative algebra results involve three-dimensional local rings, so that we can only compare localizations at height one primes of the congruence ideal and the characteristic power series of the standard (degree 5) Selmer group. The tool this time is the base change from $\mathrm{GSp}_{4}(\mathbb{Q})$ to $U(4)$ (for an imaginary quadratic field) established by C.-P. Mok [Mok14] and [Clo91] and the conclusion is that the two variable characteristic power series of the degree 5 Galois representation associated to the family $\sigma$ generates the height one part of the ideal of congruences between the base change of $\sigma$ to $U(4)$ and families on $U(4)$ which don't come from $\operatorname{GSp}_{4}(\mathbb{Q})$. See Section 7 for the statement and the proof.

\section{TheOREMS $R_{n-1}=\mathbf{T}_{n-1}^{u}$ FOR $n \geq 4$}

2.1. Big ordinary Hecke algebra for unitary groups. Recall that we fixed a squarefree integer $N=q_{1} \cdot \ldots \cdot q_{k}$ prime to $p$. As stated in Theorem 1.6, we will need to assume in some cases that $k \geq 2$. Hida theory for unitary groups [PAF, Chapt.8] is developed using coherent cohomology but hereafter we follow the presentation of [Ge10, Section 2] (see also [Ge16]) using definite forms of unitary groups. We fix an auxiliary imaginary quadratic field $K=\mathbb{Q}(\sqrt{-\Delta})$ of negative discriminant $-\Delta$ relatively prime to $N p$ such that $p=\mathfrak{p p}^{c}$ and $q_{1}=\mathfrak{q}_{1} \mathfrak{q}_{1}^{c}$ split and $q_{2}$ remains inert in $K$. Let $D$ be a central division algebra over $K$ of rank $n^{2}$ whose ramification set $S_{D}$ consists in the primes above $q_{1}$. From the calculations of [Clo91, (2.3) and Lemma 2.2], we see that

(Case 1) If $n$ is odd or is divisible by 4 , then for any $k \geq 1$, there exists an involution of second kind $*$ on $D$ which is positive definite at $\infty$ and such that the unitary group $U(D, *)$ is quasisplit at all inert places.

(Case 2) If $n=2 m$ with $m$ odd; for $k \geq 2$ there exists an involution of second kind $*$ on $D$ which is positive definite at $\infty$ and such that the unitary group $U(D, *)$ is quasisplit at all inert places except $q_{2}$.

We fix $G=U(D, *)$ as above.

Definition 2.1. We fix an auxiliary level group $U^{p}=\prod_{i=1}^{k} U_{q_{i}} \times U^{N p}$ of Iwahori type of squarefree level $N$; this means that for each prime $q$ dividing $N, U_{q}$ is

-equal to the standard Iwahori subgroup of $G_{q}$ if $G$ is quasi-split at $q$ (that is, either $G_{q}=\mathrm{GL}_{n}\left(K_{\mathfrak{q}}\right)$ if $q$ splits in $K$, or if $G_{q}$ is the quasi-split unitary group),

$-U_{q}$ is a minimal parahoric subgroup if $G_{q}$ is not quasisplit.

Remark 2.2. Let $\Pi_{G}$ be any cuspidal automorphic representation on $G$ with cohomological weight and level $U=U^{p} \times U_{p}$. Let $q$ be a prime dividing $N$ which is inert in $K$; the condition $\Pi_{G, q}^{U_{q}} \neq 0$ implies that the base change of $\Pi_{G, q}$ to $K_{q}$ has fixed vectors by the Iwahori subgroup of $G L_{n}\left(K_{q}\right)$. Let $\sigma_{\Pi_{G, q}}$ be the p-adic Weil-Deligne representation of $\Pi_{G, q}$. Let $\widetilde{\sigma}_{\Pi_{G, q}}$ be its restriction to the inertia subgroup $I_{q}$. If the reduction modulo $p$ of $\widetilde{\sigma}_{\Pi_{G, q}}$ is regular unipotent, the same holds for $\widetilde{\sigma}_{\Pi_{G, q}}$ and $\Pi_{G, q}$ is the twist of the Steinberg representation by an unramified at most quadratic character.

This remark will be useful later.

We fix an isomorphism $i_{p}: G_{p}=G\left(\mathbb{Q}_{p}\right) \cong \mathrm{GL}_{n}\left(\mathbb{Q}_{p}\right)$, which we use to identify these groups. Thus, we can view $U_{p}=i_{p}^{-1}\left(\mathrm{GL}_{n}\left(\mathbb{Z}_{p}\right)\right)$ as a hyperspecial maximal compact subgroup of $G_{p}$. From now on, we omit the mention of $i_{p}$ and we simply write $U_{p}=\mathrm{GL}_{n}\left(\mathbb{Z}_{p}\right)$. Let $I_{p} \subset U_{p}$ be the Iwahori subgroup and for $0 \leq b \leq c, I_{p}^{b, c} \subset U_{p}$ be the subgroup of matrices whose reduction modulo $p^{c}$, resp. $p^{b}$, 
belong to the group of $\mathbb{Z} / p^{c} \mathbb{Z}$-points of the subgroup $B$ of upper triangular matrices of $\mathrm{GL}_{n}$, resp. to the group of $\mathbb{Z} / p^{b} \mathbb{Z}$-points of the group $N^{\prime}=\operatorname{diag}(1, \ldots, 1, *) \cdot N^{+}$where $N^{+}$is the group of upper unipotent matrices. Note the difference with [Ge10, Def.2.1] where the condition modulo $p^{b}$ is that $u \in N^{+}\left(\mathbb{Z} / p^{b} \mathbb{Z}\right)$. Here we enlarge the group $N^{+}$to $N^{\prime}$. This is because we want to define a big ordinary Hecke algebra depending only on the semisimple variables of the diagonal torus $T$, not on the whole of $T$. Let $T^{s s}=\operatorname{Ker}\left(\operatorname{det}: T \rightarrow \mathbb{G}_{m}\right)$. We have a decomposition $T \cong T^{s s} \times \mathbb{G}_{m}$ given by $u \mapsto\left(u^{s s}\right.$, det $\left.u\right)$ where $u=\operatorname{diag}\left(u_{1}, \ldots, u_{n}\right)$ and $u^{s s}=\operatorname{diag}\left(u_{1}, \ldots, u_{n-1},\left(u_{1} \cdot \ldots \cdot u_{n-1}\right)^{-1}\right)$.

Let $G_{f}$ be the locally compact group of finite adèles of $G$ and $G_{\mathbb{Q}}$ be the subgroup of principal adèles. By compactness of $G_{\infty}, G_{\mathbb{Q}}$ is discrete in $G_{f}$ and for any compact open subgroup $U$ of $G_{f}$, the quotient $G_{\mathbb{Q}} \backslash G_{f} / U$ is finite (see [PR94, Chap.5, Section 3, Th.5.5]). We fix from now on the auxiliary level group $U=U^{p} \times U_{p}$ of Iwahori type of squarefree level $N$ in the sense of Definition 2.1. As usual, one can add another auxiliary prime $r$ (in the sense of Taylor-Wiles) prime to $N p$ to assure that $U$ is sufficiently small: $G_{\mathbb{Q}} \cap U=1$. After localization at a suitable maximal ideal, it will not introduce extra ramification at $r$ for the automorphic forms occuring in the Hida-Geraghty Hecke algebra of auxilary level $U$ defined below.

For $c \geq b \geq 0$, let $U^{b, c}=U^{p} \times I_{p}^{b, c}$. Let $E$ be a sufficiently large $p$-adic field; let $\mathcal{O}$ be its valuation ring. Any $(n-1)$-tuple $\lambda=\left(\lambda_{1}, \ldots, \lambda_{n-1}\right) \in \mathbb{Z}^{n-1}$ defines a character of the diagonal torus $T$ of $\mathrm{GL}_{n}\left(\right.$ and of $\left.T^{s s}=T \cap \mathrm{SL}_{n}\right)$ by

$$
\operatorname{diag}\left(t_{1}, \ldots, t_{n}\right) \mapsto t_{1}^{\lambda_{1}} \cdot \ldots \cdot t_{n-1}^{\lambda_{n-1}}
$$

Let us assume that $\lambda_{1} \geq \ldots \lambda_{n-1} \geq 0$, let $L_{\lambda}(\mathcal{O})$ be the "maximal" $\mathcal{O}$-representation of $\mathrm{GL}_{n}$ of highest weight $\lambda$ (see [PT02]). Let $w_{0}$ be the longest element of the Weyl group of $\mathrm{GL}_{n}$. Then $L_{\lambda}(\mathcal{O})$ is defined as the algebraic induction of $w_{0} \lambda$ from $B$ to $\mathrm{GL}_{n}$, that is, the $\mathcal{O}$-module of rational functions $\phi \in \mathcal{O}\left[\mathrm{GL}_{n}\right]$ such that $\phi\left(t n^{+} g\right)=\left(w_{0} \lambda\right)(t) \phi(g)$ for any $b=t n^{+} \in B$. We define the $\mathcal{O}$-module $S_{\lambda}\left(U^{b, c} ; \mathcal{O}\right)$ of cuspidal forms of level $U^{b, c}$ for $G$ by

$$
S_{\lambda}\left(U^{b, c} ; \mathcal{O}\right)=\left\{s: G_{\mathbb{Q}} \backslash G_{f} \rightarrow L_{\lambda}(\mathcal{O}) ; s(x u)=u_{p}^{-1} \cdot s(x) \quad \text { for any } u \in U^{b, c}\right\}
$$

For $c>0$, let $h_{\lambda}\left(U^{b, c} ; \mathcal{O}\right)$ be the $\mathcal{O}$-algebra of endomorphisms of $S_{\lambda}\left(U^{b, c} ; \mathcal{O}\right)$ generated by the Hecke operators

- $T_{\xi, i}=\left[U^{b, c} \alpha_{\varpi \xi}^{(i)} U^{b, c}\right]_{\lambda}$, where $i=1, \ldots, n, \alpha_{\varpi}^{(i)}=\left(\begin{array}{cc}\varpi_{\xi} 1_{i} & 0 \\ 0 & 1_{n-i}\end{array}\right)$, and $\xi$ runs over the degree one primes of $\mathcal{O}_{K}$, relatively prime to $M \Delta p$.

- $U_{\varpi, i}=\left(w_{0} \lambda\right)\left(\alpha_{\varpi}^{(i)}\right)^{-1}\left[U^{b, c} \alpha_{\varpi}^{(i)} U^{b, c}\right]_{\lambda}, i=1, \ldots, n-1$, where $\varpi$ is a uniformizing parameter of $\mathfrak{p}$ and $\alpha_{\varpi}^{(i)}=\left(\begin{array}{cc}\varpi 1_{i} & 0 \\ 0 & 1_{n-i}\end{array}\right)$ as before,

- $\langle u\rangle_{\lambda}=\left[U^{b, c} u U^{b, c}\right]_{\lambda}$ where $u \in T(\mathbb{Z})$ (actually, it depends only on the image of $u$ in $\left.T^{s s}\left(\mathbb{Z} / p^{b} \mathbb{Z}\right)\right)$.

Recall that $\left[U^{b, c} \alpha U^{b, c}\right]_{\lambda}$ acts by $\left(\left[U^{b, c} \alpha U^{b, c}\right]_{\lambda} \cdot s\right)(x)=\sum_{i} \alpha_{i, p} \cdot s\left(x \alpha_{i}\right)$ where $U^{b, c} \alpha U^{b, c}=\bigsqcup_{i} \alpha_{i} U^{b, c}$ (see beginning of [Ge10, Sect. 2.3]). The operators $T_{\xi, i}, U_{\varpi, i}$ and $\langle u\rangle_{\lambda}$ preserve integrality [Ge10, Def. 2.3.1 and 2.3.2].

Let $e$ be the ordinary idempotent associated to $U_{\varpi}=\prod_{i=1}^{n-1} U_{\varpi, i}$. We define

$$
h_{n-1}={\underset{\iota}{c}}_{\lim } e \cdot h_{\lambda}\left(U^{c, c} ; \mathcal{O}\right)
$$

It does not depend on the dominant weight $\lambda[\mathrm{Ge10,}$ Prop.2.6.1]. It is reduced [Ge10, Lemma 2.4.4]. Let $T_{0}=T\left(\mathbb{Z}_{p}\right), T_{0}^{s s}=T^{s s}\left(\mathbb{Z}_{p}\right) ;$ and similarly let $T_{b}=\operatorname{Ker}\left(T\left(\mathbb{Z}_{p}\right) \rightarrow T\left(\mathbb{Z} / p^{b} \mathbb{Z}\right)\right), T_{b}^{s s}=$ $\operatorname{Ker}\left(T^{s s}\left(\mathbb{Z}_{p}\right) \rightarrow T^{s s}\left(\mathbb{Z} / p^{b} \mathbb{Z}\right)\right)$. We can decompose $T_{0}=T(\mathbb{Z} / p \mathbb{Z}) \times T_{1}$ and $T_{1}=T_{1}^{s s} \times\left(1+p \mathbb{Z}_{p}\right)$. For $p>2$, let $u=1+p$. We can identify the $\mathcal{O}$-algebra $\Lambda_{n-1}$ of power series in $n-1$ variables to the completed group algebra $\mathcal{O}\left[\left[T_{1}^{s s}\right]\right]$ by sending $1+X_{i}$ to $\operatorname{diag}\left(1_{i-1}, u, 1_{n-1-i}, u^{-1}\right)$. We view $h_{n-1}$ as a $\Lambda_{n-1}$-algebra via the weight 0 diamond action $T_{1} \rightarrow h_{n-1}^{\times}, u \rightarrow\langle u\rangle_{0}$. As a $\Lambda_{n-1}$-algebra, $h_{n-1}$ is finite torsion-free. Indeed, the proof of [Ge10, Prop.2.5.3] goes through when one replaces the group $T_{b}$ by the group $T_{b}^{s s}$, because with our modified definition of the groups $U^{b, c}$, we do have

$$
S_{\lambda}\left(U^{c, c} ; \mathcal{O}\right)^{T_{b}^{s s}}=S_{\lambda}\left(U^{b, c} ; \mathcal{O}\right)
$$


hence, by Hida's lemma (see [Ge10, Lemma 2.5.2], we see that

$$
e \cdot S_{\lambda}\left(U\left(p^{\infty}\right) ; E / \mathcal{O}\right)^{T_{b}^{s s}}=e \cdot S_{\lambda}\left(U^{b, b} ; E / \mathcal{O}\right)
$$

which is the key step for the vertical control theorem and its corollary [Ge10, Coroll.2.5.4]. From this fact, the finiteness and torsion-freeness of our Hecke algebra over $\Lambda_{n-1}$ follow as in [Ge10, Coroll.2.5.4].

2.2. Symm ${ }^{n-1}$ Langlands functoriality. We assume that the Symm ${ }^{n-1}$ Langlands functoriality from $\mathrm{GL}_{2}$ to $\mathrm{GL}_{n}$ is established (sending non CM classical cusp eigensystems to cuspidal eigensystems on $\mathrm{GL}_{n}$ ). It is known for $n-1 \leq 8$ thanks to the works of Kim-Shahidi [KS02b], Kim [Kim03] and Clozel-Thorne [CT14], [CT15]. Let $\pi$ be a non CM holomorphic cuspidal representation of $\mathrm{GL}_{2}\left(\mathbf{A}_{\mathbb{Q}}\right)$ cohomological for a local system of highest weight $a \geq 0$, with conductor $N$ and level group $U_{0}^{(1)}(N)=\left\{u \in \mathrm{GL}_{2}(\widehat{\mathbb{Z}}) ; u(\bmod N)\right.$ upper triangular $\}$, (that is, $\left.\operatorname{dim} \pi_{0}^{U_{0}^{(1)}(N)}=1\right)$. The Langlands parameter $r_{\infty}: W_{\mathbb{R}} \rightarrow G L_{2}(\mathbb{C})$ of $\pi_{\infty}$ is given by $r_{\infty}(z)=\operatorname{diag}\left((z / \bar{z})^{(a+1) / 2},(\bar{z} / z)^{a+1) / 2}\right)$ for $z \in W_{\mathbb{C}}$ and $r_{\infty}(j)=\left(\begin{array}{cc}0 & 1 \\ (-1)^{a+1} & 0\end{array}\right)$.

By assumption, there is an automorphic cuspidal representation $\Pi=\operatorname{Symm}^{n-1} \pi$ on $\mathrm{GL}_{n}$. The Langlands parameter $R_{\infty}: W_{\mathbb{R}} \rightarrow G L_{n}(\mathbb{C})$ of $\Pi_{\infty}$ is given by its restriction to $W_{\mathbb{C}}$ by

$$
R_{\infty}(z)=\operatorname{diag}\left((z / \bar{z})^{(n-1)(a+1) / 2},(z / \bar{z})^{(n-3)(a+1) / 2} \ldots,(z / \bar{z})^{-(n-1)(a+1) / 2}\right) .
$$

It follows from the local Langlands correspondence for $\mathrm{GL}_{n}\left(\mathbf{A}_{\mathbb{Q}}\right)$ that $\Pi$ is Steinberg at all primes dividing $N$. Let $\Pi_{K}$ the base change of $\Pi$ to $\mathrm{GL}_{n}\left(\mathbf{A}_{K}\right)$ (see [AC89, III,5]); the Langlands parameter of $\Pi_{K, \infty}$ is $\left.R_{\infty}\right|_{W_{\mathbb{C}}}$. It is cohomological. Moreover, $\Pi_{K, \mathfrak{q}}$ is Steinberg at all primes $\mathfrak{q}$ of $K$ dividing $N$. In particular, $\Pi_{K}$ is square-integrable at both places of $S_{D}$; therefore, by the Jacquet-Langlands correspondence for $\mathrm{GL}_{n}$ (see [Vi84] and [AC89]), it descends to a cuspidal representation $\Pi_{D}$ on $D^{\times}\left(\mathbf{A}_{K}\right)$. Note that $\Pi_{D, \infty}=\Pi_{K, \infty}$ is cohomological. By [Clo91, Lemma 3.8 and Prop.4.11 ], $\Pi_{D}$ descends as a cuspidal representation $\Pi_{G}$ on $G$ (for more general results of descent from $D^{\times}$to $G$, see Labesse [Lab09, Th.5.4] and C.-P. Mok [Mok14]). The difference with [Clo91, Prop.4.11] is that here $\Pi_{G, \infty}$ is the irreducible representation of highest weight $((n-1) a,(n-2) a, \ldots, a, 0)$ of the compact group $U(n)$ (instead of being a cohomological representation of $U(n-1,1)$ ); moreover, $\Pi_{G, \mathfrak{q}}$ is Steinberg at all places $\mathfrak{q}$ of $K$ dividing $N$. Note that

- For any rational prime $q$ prime to $N p$ which splits in $K$, say, $q=\xi \xi^{c}$, the Hecke eigenvalues $t_{\xi, i}$ on the 1 -dimensional space $\Pi_{\xi}^{U_{\xi}}$ of the Hecke operators $T_{\xi, i}, i=1, \ldots, n-1$, are determined by the relation between Hecke polynomials:

$$
P_{\Pi_{\xi}}^{(n-1)}(T)=\operatorname{Sym}^{n-1} P_{\pi_{q}}^{(1)}(T) \in E[T]
$$

where

$$
\begin{gathered}
P_{\pi_{q}}^{(1)}(T)=T^{2}-a_{q} T+q^{a+1}=\left(T-\alpha_{q}\right)\left(T-\beta_{q}\right), \\
\operatorname{Sym}^{n-1} P_{\pi_{q}}^{(1)}(T)=\left(T-\alpha_{q}^{n-1}\right)\left(T-\alpha_{q}^{n-2} \beta_{q}\right) \ldots\left(T-\beta_{q}^{n-1}\right),
\end{gathered}
$$

and

$P_{\Pi_{\xi}}^{(n-1)}(T)=T^{n}-t_{\xi, 1} T^{n-1}+\ldots+(-1)^{j} q^{j(j+1) / 2} t_{\xi, j} T^{n-j}+\ldots+(-1)^{n} q^{n(n+1) / 2} t_{\xi, n}$

- if $a \neq 0$, the local component $\Pi_{\mathfrak{p}}$ is unramified and the eigenvalues $u_{\varpi, i}$ of the normalized Atkin-Lehner operators $U_{\varpi, i}(i=1, \ldots, n-1)$, on the finite dimensional vector space $\Pi_{\mathfrak{p}}^{I_{p}}$ are given by

$$
\prod_{i=1}^{n}\left(T-p^{i-1} \frac{u_{\varpi, i}}{u_{\varpi, i-1}} \varpi^{(i-1) a}\right)=\operatorname{Sym}^{n-1} P_{\pi_{p}}^{(1)}(T)
$$

where one has put $U_{\varpi, 0}=U_{\varpi, n}=$ Id. Explicitely, one has $u_{\varpi, 1}=\alpha_{p}^{n-1}, u_{\varpi, 2}=\alpha_{p}^{n-2} \frac{\beta_{p}}{p \varpi^{a}}, \ldots$, $u_{\varpi, n-1}=\alpha_{p}\left(\frac{\beta_{p}}{p \varpi^{a}}\right)^{n-2}$, where $\alpha_{p}$ is the unit root of $P_{\pi_{p}}^{(1)}(T)$. Note that the eigenvalues $u_{\varpi, i}$ are $p$-adic units since $\frac{w}{p}$ is. This follows from Lemma 2.7.5 of [Ge10] because the weight $\lambda=\left(\lambda_{1}, \ldots, \lambda_{n}\right)$ is given by $((n-1) a,(n-2) a, \ldots, a, 0)$, hence it is regular if $a \neq 0$, hence the lemma applies. 
Let $h_{1}^{N-\text { new }}$ be the $N$-new quotient of $h_{1}$. For any prime $q$ prime to $N p$ splitting in $K$ as $\xi \xi^{c}$, let

$$
P_{\xi}^{(n-1)}(T)=T^{n}-T_{\xi, 1} T^{n-1}+\ldots+(-1)^{j} q^{j(j+1) / 2} T_{\xi, j} T^{n-j}+\ldots+(-1)^{n} q^{n(n+1) / 2} T_{\xi, n}
$$

be the universal Hecke polynomial of the spherical Hecke algebra of $G L_{n}$ at $\xi$ and $P_{q}^{(1)}(T)=$ $T^{2}-T_{q} T+q S_{q}$ the universal Hecke polynomial of the spherical Hecke algebra for $\mathrm{GL}_{2}$ at $q$. Recall that $\ell: \mathbb{Z}_{p}^{\times} \rightarrow p \mathbb{Z}_{p}$ is defined by $\left.x=\omega(x) u^{\ell(x)}\right)$. We can interpolate the formulas above:

Proposition 2.3. There exists a ring homomorphism $\theta: h_{n-1} \rightarrow h_{1}$ above the algebra homomorphism $\Lambda_{n-1} \rightarrow \Lambda_{1}$ given by $1+X_{i} \rightarrow(1+X)^{n-i}$ for $i=1, \ldots, n-1$. The homomorphism $\theta$ is characterized by the fact that for any prime $q$ prime to $N p$ splitting in $K$ as $\xi \xi^{c}$, the image by $\theta$ of the universal Hecke polynomial $P_{\xi}^{(n-1)}(T)$ is $\operatorname{Sym}^{n-1} P_{q}^{(1)}(T)$, while the images $\theta\left(U_{\varpi, i}\right)$ are given by $\left(U_{p}^{(1)}\right)^{n-2 i+1} \cdot\left(\omega\left(\frac{p}{\varpi}\right)(1+X)^{\ell\left(\frac{p}{\varpi}\right)}\right)^{i-1}$. Let $\pi$ be an $N$-new p-ordinary holomorphic cuspidal automorphic form $\pi$ on $G L_{2}(\mathbb{Q})$ of highest weight $a>0$. Let $\mu_{\pi}: h_{1}^{N-n e w} \rightarrow \mathcal{O}$ be the associated eigensystem. Let $\theta_{a}=\theta\left(\bmod X-u^{a}+1\right)$. Then for any rational prime $q$ split in $K$ as $\xi \xi^{c}, \mu_{\pi} \circ \theta_{a}$ sends the universal polynomial $P_{\xi}^{(n-1)}(T)$ to $\operatorname{Sym}^{n-1} P_{\pi_{q}}(T)$ and if we put $P_{\pi_{p}}(T)=\left(T-\alpha_{p}\right)\left(T-\beta_{p}\right)$, $\operatorname{ord}_{p}\left(\alpha_{p}\right)=0$, we have $\mu_{\pi} \circ \theta_{a}\left(U_{\varpi, i}\right)=\alpha_{p}^{n-i}\left(\frac{\beta_{p}}{p \varpi^{a}}\right)^{i-1}$ for $i=1, \ldots, n-1$.

Proof. For primes $q \neq p$, the statement is obvious. For the prime $p$, for any $a \neq 0$, one gets

$$
\theta\left(U_{\varpi, i}\right) \equiv\left(U_{p}^{(1)}\right)^{n-2 i+1} \cdot\left(\frac{p}{\varpi}\right)^{a(i-1)} \quad\left(\bmod X-u^{a}+1\right) .
$$

But we have for $a \geq 0$

as desired.

$$
\alpha_{p}^{n-i}\left(\frac{\beta_{p}}{p \varpi^{a}}\right)^{i-1}=\alpha_{p}^{n-i}\left(\frac{\beta_{p}}{p^{a+1}}\right)^{i-1}\left(\frac{p}{\varpi}\right)^{a(i-1)}=\alpha_{p}^{n-2 i+1}\left(\frac{p}{\varpi}\right)^{a(i-1)}
$$

2.3. Galois representations. Let $\Gamma_{\mathbb{Q}}=\operatorname{Gal}(\overline{\mathbb{Q}} / \mathbb{Q})$ and $\Gamma_{K}=\operatorname{Gal}(\bar{K} / K)$. In this section we use notations and results of [CHT08, Sect.2.1]. Let $\mathcal{G}_{n}=\left(G L_{n} \times \mathrm{GL}_{1}\right) \rtimes\{1, j\}$ where $j^{2}=1$ and $j(g, \mu) j^{-1}=\left({ }^{t} g^{-1} \mu, \mu\right)$. It is a non connected group scheme over $\mathbb{Z}$. Let $\nu: \mathcal{G} \rightarrow \mathrm{GL}_{1}$ be the homomorphism given by $(g, \mu) \mapsto \mu$ and $\nu(j)=-1$. We have the inclusions of Lie algebras $\mathfrak{s l}_{n} \subset \mathfrak{g l}_{n} \subset \operatorname{Lie} \mathcal{G}_{n}$. Note that $a d(g, \mu)(X)=g X g^{-1}$ and $a d(j)(X)=-{ }^{t} X$. We fix a sufficiently large $p$-adic field $E$ with valuation ring $\mathcal{O}$. In this section, we consider representations $\rho: \Gamma_{K} \rightarrow \mathrm{GL}_{n}(R)$ and homomorphisms $r: \Gamma_{\mathbb{Q}} \rightarrow \mathrm{GL}_{n}(R)$ for various $\mathcal{O}$-algebras $R$. The theorem below follows from [Ge10, Proposition 2.7.2] (see also [CHT08, Proposition 3.3.4]) and [Ge10, Corollary 2.7.8].

Theorem 2.4. Let $\lambda^{\prime}$ be a dominant weight for $\mathrm{GL}_{n}$; for any cuspidal automorphic representation $\Pi_{G}^{\prime}$ of $G\left(\mathbb{A}_{\mathbb{Q}}\right)$ occuring in $e \cdot S_{\lambda^{\prime}}\left(U^{0,1}, E\right)$, there exists a continuous semisimple representation

such that

$$
\rho_{\Pi_{G}^{\prime}}: \Gamma_{K} \rightarrow \mathrm{GL}_{n}(E)
$$

(i) for q prime to $N p$ splitting in $K$ as $\xi \xi^{c}, \rho_{\Pi_{G}^{\prime}}$ is unramified at $q$ and the characteristic polynomial of $\mathrm{Frob}_{\xi}$ is $P_{\Pi_{G, \xi}^{\prime}}(T)$,

(ii) $\rho_{\Pi_{G}^{\prime}}^{c} \cong \rho_{\Pi_{G}^{\prime}}^{\vee} \chi^{1-n}$

(iii) for any prime $q$ inert in $K$ not dividing $N p, \rho_{\Pi_{G}^{\prime}}$ is unramified at $q$

(iv) if moreover $\lambda^{\prime}$ is regular, $\rho_{\Pi_{G}^{\prime}}$ is crystalline and ordinary at $\mathfrak{p}$ and $\mathfrak{p}^{c}$. For instance at $\mathfrak{p}$ :

$$
\left.\rho_{\Pi_{G}^{\prime}}\right|_{\Gamma_{K \mathfrak{p}}} \sim\left(\begin{array}{cccc}
\psi_{\mathfrak{p}, 1} & * & \cdots & * \\
& \chi^{-1} \psi_{\mathfrak{p}, 2} & \cdots & * \\
& & \ddots & \\
& & & \chi^{-n+1} \psi_{\mathfrak{p}, n}
\end{array}\right)
$$

where $\psi_{\mathfrak{p}, i} \circ \operatorname{Art}_{\mathfrak{p}}: K_{\mathfrak{p}}^{\times} \rightarrow E^{\times}$is given on $\mathcal{O}_{\mathfrak{p}}^{\times}$by $x \mapsto x^{-\lambda_{n-i+1}^{\prime}}$ and by $\psi_{\mathfrak{p}, i} \circ \operatorname{Art}_{\mathfrak{p}}(\varpi)=u_{\Pi^{\prime}, i} / u_{\Pi^{\prime}, i-1}$, $i=1, \ldots, n-1$ where $u_{\Pi^{\prime}, i}$ is the unique unit eigenvalue of $U_{\varpi, i}$ on $\left(\Pi_{G, \mathfrak{p}}^{\prime}\right)^{I_{p}}$ for $i=1, \ldots, n-1$.

In particular, denoting by Art the global Artin symbol of $K$, we have for any $x \in \mathcal{O}_{K, \mathfrak{p}}^{\times}$

$$
\operatorname{det} \rho_{\Pi_{G}^{\prime}} \circ \operatorname{Art}(x)=x^{-\sum_{i=1}^{n}\left(\lambda_{n-i+1}^{\prime}+i-1\right)} .
$$


As explained in [Ge10, Proposition 2.7.2], the key ingredient in order to apply the main result of [HT01] is Coroll.5.3 of [Lab09]. The uniqueness of the unit eigenvalue of $U_{\varpi, i}$ on $\left(\Pi^{\prime}\right)_{G, \mathfrak{p}}^{I_{p}}$ for $i=1, \ldots, n-1$ is proven in [Ge10, Lemma 2.7.5 (2)].

By the theorem 2.4, we have a perfect pairing $\langle\rangle:, E^{n} \times E^{n} \rightarrow E$ and a character $\mu=$ $\chi^{1-n}: \Gamma_{\mathbb{Q}}: \Gamma_{\mathbb{Q}} \rightarrow E^{\times}$such that

- $\langle y, x\rangle=-\mu(c)\langle x, y\rangle$

- $\left\langle\rho_{\Pi_{G}^{\prime}}(\delta) x, \rho_{\Pi_{G}^{\prime}}(c \delta c) y\right\rangle=\mu(\delta)\langle x, y\rangle$.

By [CHT08, Lemma 2.1.1], there is a bijection between "polarized representations" $(\rho, \mu,\langle\rangle$, where $\rho: \Gamma_{K} \rightarrow \mathrm{GL}_{n}(E), \mu: \Gamma_{\mathbb{Q}}: \Gamma_{\mathbb{Q}} \rightarrow E^{\times}$are homomorphisms and $\langle\rangle:, E^{n} \times E^{n} \rightarrow E$ is a perfect pairing, and homomorphisms $r: \Gamma_{\mathbb{Q}} \rightarrow \mathcal{G}_{n}(E)$. We have $-\mu(c)=(-1)^{n}$. One can take the pairing to be $\operatorname{Symm}^{n-1}$ of the standard pairing on $E^{2}$ given by $J_{2}=\left(\begin{array}{cc}0 & -1 \\ 1 & 0\end{array}\right)$. It is therefore given by $\langle x, y\rangle={ }^{t} x J_{n} y$ where $J_{n}=\operatorname{Symm}^{n-1} J_{2}$ is antidiag $\left(1,-1, \ldots,(-1)^{n-1}\right)$. Therefore, Theorem 2.4 yields

Corollary 2.5. For $\Pi_{G}^{\prime}$ as above, there exists a continuous homomorphism

$$
R_{\Pi_{G}^{\prime}}: \Gamma_{\mathbb{Q}} \rightarrow \mathcal{G}_{n}(E)
$$

such that

- for $\delta \in \Gamma_{K}$, one has $R_{\Pi_{G}^{\prime}}(\delta)=\left(\rho_{\Pi_{G}^{\prime}}(\delta), \chi^{1-n}(\delta)\right)$,

- $R_{\Pi_{G}^{\prime}}(c)=\left(J_{n}^{-1},(-1)^{n}\right) j$.

Let $\pi$ be an $N$-new $p$-ordinary cuspidal holomorphic representation of level $N$ cohomological of highest weight $a \geq 0$ occuring in the Hida family $\mu$. Let $\rho_{\pi}: \Gamma_{\mathbb{Q}} \rightarrow \mathrm{GL}_{2}(\mathcal{O})$ its $p$-adic Galois representation. Assume that the residual representation $\bar{\rho}=\bar{\rho}_{\pi}: \Gamma_{\mathbb{Q}} \rightarrow \mathrm{GL}_{2}(k)$ has big image, in the sense that there exists a subfield $k^{\prime} \subset k$ such that

$$
\mathrm{SL}_{2}\left(k^{\prime}\right) \subset \operatorname{Im} \bar{\rho}_{\pi} \subset \mathrm{GL}_{2}\left(k^{\prime}\right) .
$$

Note that up to conjugation the restriction of $\bar{\rho}_{\pi}$ to a decomposition group $D_{p}$ at $p$ is given by

$$
\left(\begin{array}{cc}
\operatorname{unr}(\bar{\alpha}) & * \\
\mathbf{0} & \operatorname{unr}\left(\bar{\alpha}^{-1}\right) \omega^{-a-1}
\end{array}\right)
$$

where $\alpha=\mu\left(U_{p}\right)$.

Let $\Pi=\operatorname{Symm}^{n-1} \pi$ be the $n-1$-symmetric power cuspidal representation of $\pi$ on $\mathrm{GL}_{n}(\mathbb{Q})$ and $\Pi_{G}$ its base change to $G$. Let

$$
R_{\Pi}=\operatorname{Symm}^{n-1} \rho_{\pi}: \Gamma_{\mathbb{Q}} \rightarrow \mathrm{GL}_{n}(\mathcal{O})
$$

be the Galois representation associated to $\Pi$.

By [CHT08, Lemma 2.1.2], the continuous homomorphism

$$
R_{\Pi_{G}}: \Gamma_{\mathbb{Q}} \rightarrow \mathcal{G}_{n}(\mathcal{O})
$$

associated to $\Pi_{G}$ is given as follows. Let $c$ be a complex conjugation in $\Gamma_{\mathbb{Q}}$. For $\sigma \in \Gamma_{K}$, we put

$$
R_{\Pi_{G}}(\sigma)=\left(R_{\Pi}(\sigma),\left(\operatorname{det} \rho_{\pi}(\sigma)\right)^{n-1}\right)
$$

and for $\sigma \in \Gamma_{\mathbb{Q}} \backslash \Gamma_{K}$, and $J_{n}=\operatorname{Symm}^{n-1}\left(\begin{array}{cc}0 & -1 \\ 1 & 0\end{array}\right)$ (so that $\left.J_{n}=\operatorname{antidiag}\left(1,-1,1, \ldots,(-1)^{n-1}\right)\right)$.

Then we put

Moreover, we have

$$
R_{\Pi_{G}}(\sigma)=\left(R_{\Pi}(\sigma) J^{-1},(-1)^{n-1}\left(\operatorname{det} \rho_{\pi}(\sigma)\right)^{n-1}\right) j .
$$

$$
\nu \circ R_{\Pi_{G}}=\delta^{n} \cdot\left(\operatorname{det} R_{\Pi}\right)^{n-1} .
$$

where $\delta: \Gamma_{\mathbb{Q}} / \Gamma_{K} \cong\{ \pm 1\}$. It is ordinary at $\mathfrak{p}$ and each prime $\mathfrak{q}$ of $K$ dividing $N$, its restriction to the inertia subgroup $I_{\mathfrak{q}}$ is regular unipotent.

Note that by our assumption, there exists a subfield $k^{\prime} \subset$ such that

$$
\mathrm{Symm}^{n-1} \mathrm{SL}_{2}\left(k^{\prime}\right) \subset \operatorname{Im} \bar{R}_{\Pi} \subset k^{\prime \times} \cdot \operatorname{Symm}^{n-1} \mathrm{GL}_{2}\left(k^{\prime}\right)
$$


This implies that the residual image of $R_{\Pi}$ is big in the sense of [CHT08, Definition 2.5.1] (see [CHT08, Lemma 2.5.4] for details). Let $\mathfrak{m}$ be the maximal ideal of $h_{n-1}$ associated to the residual representation

$$
\bar{R}=\bar{R}_{\Pi_{G}}: \Gamma_{\mathbb{Q}} \rightarrow \mathcal{G}_{n}(k) .
$$

We fix a decomposition group $D_{p}=\Gamma_{K_{\mathfrak{p}}}$ at $p$ in $\Gamma_{\mathbb{Q}}$. Note that after a given conjugation, one can assume that the restriction to $D_{p}$ is upper triangular, with diagonal

which we rewrite as

$$
\operatorname{diag}\left(\operatorname{unr}(\bar{\alpha})^{n-1}, \operatorname{unr}(\bar{\alpha})^{n-2} \omega^{-a-1}, \ldots, \omega^{-(n-1)(a+1)}\right)
$$

$$
\operatorname{diag}\left(\bar{\psi}_{1}, \bar{\psi}_{2} \omega^{-1}, \ldots, \bar{\psi}_{n} \omega^{-(n-1)}\right) .
$$

Let $\mathbf{T}_{n-1}$ be the localization of $h_{n-1}$ at $\mathfrak{m}$ and, for any weight $\lambda^{\prime} \in \mathbb{Z}_{+}^{n}$ congruent to $((n-1) a,(n-$ 2) $a, \ldots, a, 0)$ modulo $p-1$, let $\mathbf{T}_{\lambda^{\prime}}\left(U^{0,1}, \mathcal{O}\right)$ be the image of $\mathbf{T}_{n-1}$ in $\operatorname{End}_{\mathcal{O}}\left(e \cdot S_{\lambda^{\prime}}\left(U^{0,1}, E\right)\right)$ We shall compare, under certain assumptions, $\mathbf{T}_{n-1}$, resp. $\mathbf{T}_{\lambda^{\prime}}\left(U^{0,1}, \mathcal{O}\right)$, with the universal ordinary deformation ring $R_{n-1}$, resp. and $R_{\lambda^{\prime}}$ of the representation $\bar{R}$ defined as follows. Let $\mathrm{CNL}_{\mathcal{O}}$ be the category of complete noetherian local $\mathcal{O}$-algebras $A$ with residue field $k=\mathcal{O} / \varpi_{E} \mathcal{O}$. For an object $A$ of $\mathrm{CNL}_{\mathcal{O}}$, a lifting $r: \Gamma_{\mathbb{Q}} \rightarrow \mathcal{G}_{n}(A)$ of $\bar{R}$ is a continuous homomorphism such that $r\left(\bmod \mathfrak{m}_{A}\right)=\bar{R}$. Two liftings $r, r^{\prime}: \Gamma_{\mathbb{Q}} \rightarrow \mathcal{G}_{n}(A)$ of $\bar{R}$ are equivalent if there exists $g \in 1+\mathfrak{m}_{A} M_{n}(A)$ such that $r^{\prime}=g \cdot r \cdot g^{-1}$. We consider the functors of liftings $\mathcal{D}$ and $\mathcal{D}_{\lambda^{\prime}}$ from $\mathrm{CNL}_{\mathcal{O}}$ to Sets defined as follows:

- The functor $\mathcal{D}$ sends an object $A$ to the set of equivalence classes of liftings $r: \Gamma_{\mathbb{Q}} \rightarrow \mathcal{G}_{n}(A)$ of $\bar{R}$ which satisfy the two following conditions

1) $r$ is $N$-minimal: for each prime $q$ dividing $N$ there exists $g_{q} \in \mathbf{1}_{n}+\mathfrak{m}_{A} \cdot M_{n}(A)$ such that for any $\sigma \in I_{q}$,

where

$$
\operatorname{pr}_{1} \circ r(\sigma)=g_{q} \cdot \exp \left(t_{p}(\sigma) N_{n}\right) \cdot g_{q}^{-1}
$$

$$
N_{n}=\left(\begin{array}{cccc}
0 & 1 & 0 & \ldots \\
0 & 0 & 1 & \ldots \\
0 & 0 & \ddots & \\
0 & 0 & \ldots & 1 \\
0 & 0 & \ldots & 0
\end{array}\right)
$$

2) $\left.r\right|_{D_{p}}$ is ordinary, that is, there exist characters $\psi_{r, 1}, \ldots, \psi_{r, n}: \Gamma_{K_{\mathfrak{p}}} \rightarrow A^{\times}$and $g \in$ $1_{n}+\mathfrak{m}_{A} M_{n}(A)$ such that for any $\sigma \in \Gamma_{K_{\mathfrak{p}}}$,

$$
\operatorname{pr}_{1} \circ r(\sigma)=g \cdot\left(\begin{array}{cccc}
\psi_{r, 1}(\sigma) & * & \ldots & * \\
& \chi^{-1} \psi_{r, 2}(\sigma) & \cdots & \\
& & \ddots & \\
& & & \chi^{-n+1} \psi_{r, n}(\sigma)
\end{array}\right) \cdot g^{-1}
$$

with the condition that for any $j=1, \ldots n, \psi_{r, j}$ is a lifting of $\bar{\psi}_{r, j}$.

- The functor $\mathcal{D}_{\lambda^{\prime}}$ is defined similarly, replacing condition 2) by the stronger condition 2) $)_{\lambda^{\prime}}$ $\left.r\right|_{D_{p}}$ is ordinary with characters $\psi_{r, j}$ lifting of $\bar{\psi}_{j}(j=1, \ldots, n)$ and for any $j=1, \ldots n$ and for any $x \in \mathcal{O}_{\mathfrak{p}}^{\times}$,

$$
\psi_{r, j}\left(\operatorname{Art}_{\mathfrak{p}}(x)\right)=x^{-\lambda_{n-j+1}^{\prime}} .
$$

The functor $\mathcal{D}$, resp. $\mathcal{D}_{\lambda^{\prime}}$ is the functor of $N$-minimal ordinary, resp. $N$-minimal ordinary of weight $\lambda^{\prime}$ deformations of $\bar{R}$. Note that condition that the characters $\psi_{r, j}$ are liftings of $\bar{\psi}_{r, j}$ implies that $\left.\psi_{r, j}\right|_{I_{p}}$ is a lifting of $\omega^{-(j-1)(a+1)}(j=1, \ldots, n)$.

Let $c_{n}$ be the least common multiple of all integers $k$ less than $n$.

Let us consider the following conditions

1a) $\alpha^{2 c_{n-1}} \not \equiv 1\left(\bmod \varpi_{E}\right)$ holds,

1b) $(n-1)(a+1)<p-1$ holds.

Condition 1a) implies that the characters $\bar{\psi}_{j}: D_{p} \rightarrow k^{\times}$associated to $\left.\bar{R}\right|_{D_{p}}$ are mutually distinct on the Frobenius element $\left[p, \mathbb{Q}_{p}\right]$, while condition $1 \mathrm{~b}$ ) implies that the restrictions to the inertia subgroup of the characters $\bar{\psi}_{j}$ are mutually distinct. In the following subsections devoted to the 
proof that $R_{n-1} \cong \mathbf{T}_{n-1}^{u}$, assumption 1a) is assumed (although we could have assumed 1b) instead). However, in the application of this theorem to the determination of the Selmer group in later sections, assumption 1b) seems unavoidable to the best of our knowledge, and will therefore be assumed.

Lemma 2.6. Assuming condition 1a) or 1b), the functors $\mathcal{D}$ and $\mathcal{D}_{\lambda^{\prime}}$ are representable by universal couples $\left(R_{n-1}, r^{\text {univ }}\right)$ and $\left(R_{\lambda^{\prime}}, r_{\lambda^{\prime}}^{\text {univ }}\right)$ where $R_{n-1}$ and $R_{\lambda^{\prime}}$ are objects of $\mathrm{CNL}_{\mathcal{O}}$ and $r^{\text {univ }}: \Gamma_{\mathbb{Q}} \rightarrow$ $\mathcal{G}_{n}\left(R_{n-1}\right)$ and $r_{\lambda^{\prime}}^{\text {univ }}: \Gamma_{\mathbb{Q}} \rightarrow \mathcal{G}_{n}\left(R_{\lambda^{\prime}}\right)$ are continuous homomorphisms such that $\mathrm{pr}_{1}$ or ${ }^{\text {univ }}$, resp. $r_{\lambda^{\prime}}^{\text {univ }}$ is conjugate in $1+\mathfrak{m}_{R_{n-1}} M_{n}\left(R_{n-1}\right)$, resp. in $1+\mathfrak{m}_{R_{\lambda^{\prime}}} M_{n}\left(R_{\lambda^{\prime}}\right)$, to

$$
\left(\begin{array}{cccc}
\psi_{1}^{\text {univ }}(\sigma) & * & \cdots & * \\
& \chi^{-1} \psi_{2}^{\text {univ }}(\sigma) & \cdots & \\
& & \ddots & \\
& & & \chi^{-n+1} \psi_{n}^{\text {univ }}(\sigma)
\end{array}\right)
$$

with $\psi_{j}^{\text {univ }}$ lifting $\bar{\psi}_{j}(j=1, \ldots, n)$, resp.

$$
\left(\begin{array}{cccc}
\psi_{\lambda^{\prime}, 1}^{\text {univ }}(\sigma) & * & \ldots & * \\
& \chi^{-1} \psi_{\lambda^{\prime}, 2}^{\text {univ }}(\sigma) & \cdots & \\
& & \ddots & \\
& & & \chi^{-n+1} \psi_{\lambda^{\prime}, n}^{\text {univ }}(\sigma)
\end{array}\right)
$$

with the same lifting condition, and such that the restriction of $\psi_{\lambda^{\prime}, j}^{\text {univ }} \circ \operatorname{Art}_{\mathfrak{p}}$ to $\mathcal{O}_{\mathfrak{p}}^{\times}$is given by $x \mapsto x^{-\lambda_{n-j+1}^{\prime}}(j=1, \ldots, n)$.

Proof. As noted above, the restriction of $\operatorname{pr}_{1} \circ \bar{R}$ to the decomposition group $D_{p}$ at $p$ is upper triangular and its diagonal is given by $\operatorname{diag}\left(\operatorname{unr}(\bar{\alpha})^{n-1}, \operatorname{unr}(\bar{\alpha})^{n-3} \omega^{-a-1}, \ldots, \operatorname{unr}(\bar{\alpha})^{-n+1} \omega^{-(n-1)(a+1)}\right)$. Hence either assumption 1a), resp. 1b), assures that the characters on the diagonal are mutually distinct on $D_{p}$, resp. $I_{p}$. This is well known to assure that the functors $\mathcal{D}$ and $\mathcal{D}_{\lambda^{\prime}}$ satisfy Schlessinger's criterion for representability (see for instance [Ti02]).

The ring $R_{n-1}$ has a natural structure of $\Lambda_{n-1}$-algebra given by the characters $\psi_{i}^{\text {univ }}: K_{\mathfrak{p}}^{\times} \rightarrow$ $R_{n-1}^{\times}$. More precisely, by identifying $\mathbb{Z}_{p}=\mathcal{O}_{\mathfrak{p}}$, we view the topological generator $u$ of $1+p \mathbb{Z}_{p}$ as topological generator of $1+\mathfrak{p}$. we then define the structural morphism $\Lambda_{n-1} \rightarrow R_{n-1}$ by sending $1+X_{i}$ to $\psi_{n-i+1}^{\text {univ }} \circ \operatorname{Art}_{\mathfrak{p}}(u)^{-1}$ for $i=1, \ldots, n-1$. Note that $\psi_{1}$ is determined by the determinant relation $\prod_{i=1}^{n} \chi^{-i+1} \psi_{i}=\chi^{-n(n+1) / 2}$.

Let $P_{\lambda^{\prime}}$ be the prime ideal of $\Lambda_{n-1}$ defined as the kernel of the morphism

$$
T_{1}^{s s} \rightarrow \mathcal{O}^{\times}, \quad \operatorname{diag}\left(t_{1}, \ldots, t_{n}\right) \mapsto t_{1}^{\lambda_{1}^{\prime}} \cdot \ldots \cdot t_{n}^{\lambda_{n}^{\prime}} .
$$

Lemma 2.7. For any $\lambda^{\prime}$ congruent to $((n-1) a,(n-2) a, \ldots, a, 0)$ modulo $p-1$, the natural ring homomorphism

induces an isomorphism

$$
R_{n-1} \rightarrow R_{\lambda^{\prime}}
$$

$$
R_{n-1} / P_{\lambda^{\prime}} R_{n-1} \cong R_{\lambda^{\prime}}
$$

Proof. It suffices to check for each $j=1, \ldots, n$ that $\psi_{j}^{\text {univ }} \circ \operatorname{Art}_{\mathfrak{p}}$ modulo $P_{\lambda^{\prime}}$ is given on $\mathcal{O}_{\mathfrak{p}}^{\times}$by $x \mapsto x^{-\lambda_{n-j+1}^{\prime}}$. This is the case on $1+\mathfrak{p}$ by definition of $P_{\lambda^{\prime}}$. This is also the case on $\mathcal{O}_{\mathfrak{p}, \text { tors }}^{\times}=\mu_{p-1}$ since $\psi_{j}^{\text {univ }}$ is a lifting of $\bar{\psi}_{j}$.

Proposition 2.8. There is a unique lifting $R^{h}: \Gamma_{\mathbb{Q}} \rightarrow \mathcal{G}\left(\mathbf{T}_{n-1}^{u}\right)$, resp. $R_{\lambda^{\prime}}^{h}: \Gamma_{\mathbb{Q}} \rightarrow \mathcal{G}_{n}\left(\mathbf{T}_{\lambda^{\prime}}\left(U^{0,1}, \mathcal{O}\right)\right)$ of $\bar{R}$ such that for any Hecke eigensystem $\theta_{\Pi_{G}^{\prime}}: \mathbf{T}_{n-1}^{u} \rightarrow \mathcal{O}^{\prime}$, resp. $\theta_{\Pi_{G}^{\prime}}: \mathbf{T}_{\lambda^{\prime}}\left(U^{0,1}, \mathcal{O}\right) \rightarrow \mathcal{O}^{\prime}$ associated to a cuspidal representation $\Pi_{G}^{\prime}$ on $G$, one has $\theta_{\Pi_{G}^{\prime}} \circ R^{h}=R_{\Pi_{G}^{\prime}}$. By universal property, the homomorphism $R^{h}$ gives rise to a surjective $\Lambda_{n-1}$-algebra homomorphism $\phi_{R^{h}}: R_{n-1} \rightarrow \mathbf{T}_{n-1}^{u}$. Similarly, the homomorphism $R_{\lambda^{\prime}}^{h}$ gives rise to a surjective $\mathcal{O}$-algebra homomorphism $\phi_{R_{\lambda^{\prime}}^{h}}: R_{\lambda^{\prime}} \rightarrow$ $\mathbf{T}_{\lambda^{\prime}}\left(U^{0,1}, \mathcal{O}\right)$ 
Proof. The existence of $R^{h}$ resp. $R_{\lambda^{\prime}}^{h}$ follows from [Ge10, Prop.2.74]. Its restriction to the inertia group $I_{q}$ at any prime divisor $q$ of $N$ is regular unipotent by Remark 2.2. Its ordinarity at $p$ follows from [Ge10, Cor.3.1.4]. This gives rise to ordered characters $\psi_{i}^{h}, i=1, \ldots, n$ lifting the ordered characters $\bar{\psi}_{i}^{h}, i=1, \ldots, n$ and such that

$$
\left.p r_{1} \circ R^{h}\right|_{\Gamma_{K_{\mathfrak{p}}}} \sim\left(\begin{array}{cccc}
\psi_{1}^{h} & * & \cdots & * \\
& \chi^{-1} \psi_{2}^{h}(\sigma) & \cdots & \\
& & \ddots & \\
& & & \chi^{-n+1} \psi_{n}^{h}(\sigma)
\end{array}\right) .
$$

Actually, viewing the topological generator $u$ of $1+p \mathbb{Z}_{p}$ as topological generator of $1+\mathfrak{p}$, we have $\psi_{n-i+1}^{h} \circ \operatorname{Art}_{\mathfrak{p}}(u)=\left(1+X_{i}\right)^{-1}$ as the series $\left(1+X_{i}\right)^{-1}$ interpolates the values $u^{-\lambda_{i}^{\prime}}$ at $X_{i}=u^{\lambda_{i}^{\prime}}$. The $N$-minimality of $R^{h}$ follows from that of $R_{\Pi_{G}^{\prime}}$ for all $\Pi_{G}^{\prime}$ 's occuring in $\mathbf{T}_{n-1}^{u}$. By universal property, this yields the existence of a unique ring homomorphism $\phi_{R^{h}}: R_{n-1} \rightarrow \mathbf{T}_{n-1}^{u}$ such that $\phi_{R^{h}} \circ R^{\text {univ }} \sim R^{h}$. The relation $\phi_{R^{h}} \circ \psi_{i}^{\text {univ }}=\psi_{i}^{h}$ implies that $\phi_{R^{h}}$ is $\Lambda_{n-1}$-linear.

The surjectivity of $\phi_{R^{h}}$ and $\phi_{R_{\lambda^{\prime}}^{h}}$ follows from the absolute irreducibility of $\bar{R}$ and Carayol's theorem : $\mathbf{T}_{n-1}^{u}=\Lambda_{n-1}\left[\left.\operatorname{Tr} R^{h}\right|_{\Gamma_{K}}\right]$ and $R_{n-1}=\Lambda_{n-1}\left[\left.\operatorname{Tr} R^{u n i v}\right|_{\Gamma_{K}}\right]$ hence $\mathbf{T}_{n-1}^{u}=\phi_{R^{h}}\left(R_{n-1}\right)$. Similarly for $\phi_{R^{h}}$

Let $\lambda^{\prime} \in \mathbb{Z}_{+}^{n}$ be an arbitrary regular dominant weight congruent to $((n-1) a,(n-2) a, \ldots, a, 0)$ modulo $p-1$. We shall use the technique of classical Taylor-Wiles systems to prove that $\phi_{R_{\lambda^{\prime}}^{h}}$ is an isomorphism and that the rings $R_{\lambda^{\prime}}$ and $\mathbf{T}_{\lambda^{\prime}}$ are local complete intersection over $\mathcal{O}$. From this it will be easy by varying $\lambda^{\prime}$ to deduce that $\phi_{R^{h}}$ is an isomorphism and that $R_{n-1}$ and $\mathbf{T}_{n-1}^{u}$ are local complete intersection over $\Lambda_{n-1}$.

We follow (in an easier situation) the proof of [Ge10, Section 3] which itself relies on calculations of [CHT08, Section 3.5].

2.4. Galois cohomology. Let $p>2$ and $\bar{R}=\operatorname{Symm}^{n-1} \bar{\rho}_{\pi}$ where $\pi$ is holomorphic cuspidal on $G L_{2}(\mathbb{Q})$, of square free conductor $N$ and cohomological for a local system of highest weight $a \geq 0$. We assume it is $p$-ordinary. Therefore it occurs in a (unique) Hida family $\mu$. We assume that $\bar{\rho}_{\pi}$ has big image and that it is $N$-minimal as above. We also assume 1a).

Note that the image $\bar{R}(c)$ of the complex conjugation $c$ is conjugate in $\mathcal{G}_{n}(k)$ to $\left(J_{n}^{-1},(-1)^{n}\right) j$ where $J_{n}=\operatorname{antidiag}\left(1,-1, \ldots, 1,(-1)^{n-1}\right)$ From this we have as in [CHT08, Lemma 2.1.3] :

Lemma 2.9. $\operatorname{dim}_{k}\left(\mathfrak{g l}_{n}\right)^{c=1}=n(n-1) / 2$.

Proof. For $X \in M_{n}(k)$, we have $\operatorname{Ad} \bar{R}(c)(X)=J_{n}^{-1} j X j^{-1} J_{n}$. We have $J_{n}^{-1}=(-1)^{n-1} J_{n}={ }^{t} J_{n}$ and $j X j^{-1}=-{ }^{t} X$, hence

Ad $\bar{R}(c)(X)=X$ if and only if $J_{n}^{-1} X$ is antisymmetric. The subspace of these matrices has dimension $n(n-1) / 2$.

Let $M=\mathfrak{g l}_{n}(k)=\operatorname{Ad}_{\mathfrak{g l}_{n}} \bar{R}$ and $M^{*}=\operatorname{Hom}_{k}(M, k(1))$ its $k$-Cartier dual. For $N=M$ or $M^{*}$, let $h^{0}(N)=\operatorname{dim}_{k} \mathrm{H}^{0}\left(\Gamma_{\mathbb{Q}}, N\right)$; for any place $v$ of $\mathbb{Q}$, and any fixed decomposition group $D_{v} \subset \Gamma_{\mathbb{Q}}$, let $h_{v}^{i}(M)=\operatorname{dim}_{k} \mathrm{H}^{i}\left(D_{v}, M\right)(i=0,1,2)$. Let $Q=\left\{q_{1}, \ldots, q_{r}\right\}$ be a finite set of primes disjoint of those dividing $N p$ such that for any $i=1, \ldots, r, q_{i}=v_{i} v_{i}^{c}$ splits in $K$. For any finite place $v \neq p$ of $\mathbb{Q}$ with $v \notin Q$, let

$$
L_{Q, v}=L_{v}(M)=\mathrm{H}_{\mathrm{unr}}^{1}\left(\Gamma_{v}, M\right)=\operatorname{Ker}\left(\mathrm{H}^{1}\left(\Gamma_{v}, M\right) \rightarrow \mathrm{H}^{1}\left(I_{v}, M\right)\right)=\operatorname{Ker}\left(\mathrm{H}^{1}\left(D_{v} / I_{v}, M^{I_{v}}\right)\right.
$$

for each $v \in Q$, let $L_{Q, v} \subset \mathrm{H}^{1}\left(D_{v}, M\right)$ to be specified later in such a way that $\operatorname{dim}_{k} L_{Q, v}-h_{v}^{0}(M)=1$. We also put $L_{\infty}=0$ and

$$
L_{Q, p}=L_{p}(M)=\operatorname{Im}\left(L_{p}^{\prime}(M) \rightarrow \mathrm{H}^{1}\left(\Gamma_{p}, M\right)\right.
$$

where $\left.\left.L_{p}^{\prime}(M)=\operatorname{Ker}\left(\mathrm{H}^{1} \Gamma_{p}, F^{0} M\right) \rightarrow \mathrm{H}^{1} \Gamma_{p}, F^{0}(M) / F^{1}(M)\right)\right)$.

For any place $v$ of $\mathbb{Q}$, let $L_{Q, v}^{\perp}$ be the orthogonal in $\mathrm{H}^{1}\left(D_{v}, M^{*}\right)$ of $L_{Q, v} \subset \mathrm{H}^{1}\left(D_{v}, M\right)$ for the local Tate duality $\mathrm{H}^{1}\left(D_{v}, M\right) \times \mathrm{H}^{1}\left(D_{v}, M^{*}\right) \rightarrow k$. When $L_{Q, v}=\mathrm{H}_{\mathrm{unr}}^{1}\left(\Gamma_{v}, M\right)$, one has $L_{Q, v}^{\perp}=$ $\mathrm{H}_{\mathrm{unr}}^{1}\left(\Gamma_{v}, M^{*}\right)$. Moreover it is easy to check that $L_{p}(M)^{\perp}=L_{p}\left(M^{*}\right)$ associated to the $p$-ordinarity 
filtration of $M^{*}$ given by $F^{i}\left(M^{*}\right)=\left(F^{b-a+1-i}(M)\right)^{\perp}$ where $a$, resp. $b$ is the smallest, resp. largest weight of $F^{\bullet}(M)$ (that is, $F^{a}(M)=M \neq F^{a+1}(M)$, and $F^{b}(M) \neq 0$ but $F^{b+1}(M)=0$ ). Let $\mathcal{L}_{Q}=\left(L_{Q, v}\right)_{v}$ and $\mathcal{L}_{Q}^{\perp}=\left(L_{Q, v}^{\perp}\right)_{v}$. We define the Selmer groups

$$
\mathrm{H}_{\mathcal{L}_{Q}}^{1}(M)=\operatorname{Ker}\left(\mathrm{H}^{1}(\Gamma, M) \rightarrow \bigoplus_{v} \mathrm{H}^{1}\left(D_{v}, M\right) / L_{Q, v}\right)
$$

and

$$
\mathrm{H}_{\mathcal{L}_{\bar{Q}}^{\perp}}^{1}\left(M^{*}\right)=\operatorname{Ker}\left(\mathrm{H}^{1}\left(\Gamma, M^{*}\right) \rightarrow \bigoplus_{v} \mathrm{H}^{1}\left(D_{v}, M^{*}\right) / L_{Q, v}^{\perp}\right)
$$

They are finite and their cardinalities are denoted by $h_{\mathcal{L}_{Q}}^{1}(M)$ resp. $h_{\mathcal{L}_{\bar{Q}}^{\perp}}^{1}\left(M^{*}\right)$. The Poitou-Tate Euler characteristic formula, as formulated for instance in [DDT94, Theorem 2.18], yields

$$
h_{\mathcal{L}_{Q}}^{1}(M)-h_{\mathcal{L}_{Q}^{\perp}}^{1}\left(M^{*}\right)=h^{0}(M)-h^{0}\left(M^{*}\right)+\sum_{v}\left(\operatorname{dim}_{k} L_{Q, v}-h_{v}^{0}(M)\right) .
$$

Proposition 2.10. We have:

(i) $\operatorname{dim}_{k} L_{Q, \ell}-h_{\ell}^{0}(M)=0$ for any $\ell \notin Q$ and $\ell \neq p$,

(ii) $\operatorname{dim}_{k} L_{Q, q}-h_{q}^{0}(M)=1$ for $q \in Q$,

(iii) $\operatorname{dim}_{k} L_{Q, p}-h_{p}^{0}(M) \leq n(n-1) / 2$, (this uses 1a) and 1b)).

(iv) $h_{\infty}^{0}=h^{0}\left(D_{\infty}, M\right)=n(n-1) / 2$

(v) $h^{0}(M)=0=h^{0}\left(M^{*}\right)=0$.

It follows that

$$
h_{\mathcal{L}_{Q}}^{1}(M)-h_{\mathcal{L}_{\bar{Q}}^{\perp}}^{1}\left(M^{*}\right) \leq \sharp Q=r .
$$

Proof. From the Poitou-Tate Euler characteristic formula, the last inequality follows from the four first formulas. The first equality is clear from the exact sequence

$$
0 \rightarrow \mathrm{H}^{0}\left(D_{\ell}, M\right) \rightarrow M^{I_{\ell}} \stackrel{F_{\ell}-1}{\rightarrow} M^{I_{\ell}} \rightarrow \mathrm{H}^{1}\left(D_{\ell} / I_{\ell}, M^{I_{\ell}}\right) \rightarrow 0 .
$$

Let us check the inequality at $p$. We proceed as in [GeTi05, Lemma 10.4.4]. Let $\mathfrak{b}_{n}, \mathfrak{n}_{n}, \mathfrak{t}_{n}$ be the Lie algebras of the upper triangular, upper unipotent subgroup, resp. of their quotient. We have an exact sequence

$$
\left.\left.\mathrm{H}^{0}\left(D_{p}, \mathfrak{n}_{n}\right) \rightarrow \mathrm{H}^{0}\left(D_{p}, \mathfrak{b}_{n}\right) \rightarrow \mathrm{H}^{0}\left(D_{p}, \mathfrak{b}_{n} / \mathfrak{n}_{n}\right)\right) \rightarrow \mathrm{H}^{1}\left(D_{p}, \mathfrak{n}_{n}\right) \rightarrow \mathrm{H}^{1}\left(D_{p}, \mathfrak{b}_{n}\right) \rightarrow \mathrm{H}^{1}\left(D_{p}, \mathfrak{b}_{n} / \mathfrak{n}_{n}\right)\right)
$$

Moreover, we also have an exact sequence

$$
0 \rightarrow \mathrm{H}^{1}\left(D_{p} / I_{p}, \mathfrak{b}_{n} / \mathfrak{n}_{n}\right) \rightarrow \mathrm{H}^{1}\left(D_{p}, \mathfrak{b}_{n} / \mathfrak{n}_{n}\right) \rightarrow \mathrm{H}^{1}\left(I_{p}, \mathfrak{b}_{n} / \mathfrak{n}_{n}\right)
$$

By assumptions 1a) and 1b), we have $h^{0}\left(D_{p}, \mathfrak{n}_{n}\right)=0$ and $h^{2}\left(D_{p}, \mathfrak{n}_{n}\right)=h^{0}\left(D_{p}, \mathfrak{n}_{n}^{\vee}(1)\right)=0$. Therefore, we have $L_{p}(M)^{\prime}=L_{p}(M)$ and

$$
h^{0}\left(D_{p}, \mathfrak{b}_{n}\right)-h^{0}\left(D_{p}, \mathfrak{b}_{n} / \mathfrak{n}_{n}\right)+h^{1}\left(D_{p}, \mathfrak{n}\right)-\operatorname{dim}_{k} L_{Q, p}+h^{1}\left(D_{p} / I_{p}, \mathfrak{b}_{n} / \mathfrak{n}_{n}\right)=0
$$

By cyclicity of $D_{p} / I_{p}$, we have $h^{0}\left(D_{p}, \mathfrak{b}_{n} / \mathfrak{n}_{n}\right)=h^{1}\left(D_{p} / I_{p}, \mathfrak{b}_{n} / \mathfrak{n}_{n}\right)$. Moreover By Tate local duality, we have

$$
h^{0}\left(D_{p}, \mathfrak{n}_{n}\right)-h^{1}\left(D_{p}, \mathfrak{n}_{n}\right)+h^{2}\left(D_{p}, \mathfrak{n}_{n}\right)=-\operatorname{dim}_{k} \mathfrak{n}_{n}
$$

Hence $h^{1}\left(D_{p}, \mathfrak{n}_{n}\right)=\operatorname{dim}_{k} \mathfrak{n}_{n}$. We conclude that $\operatorname{dim}_{k} L_{Q, p}-h^{0}\left(D_{p}, \mathfrak{b}_{n}\right)=\operatorname{dim}_{k} \mathfrak{n}_{n}=n(n-1) / 2$. It implies $\operatorname{dim} L_{Q, p}-h^{0}\left(D_{p}, \mathfrak{g l}_{n}\right) \leq n(n-1) / 2$ as desired. Satement (iv) follows from 2.9. Statement (v) follows from the fact that $M$ (and $M^{*}$ ) is the sum of the irreducible $\Gamma$-modules $\overline{\mathcal{A}}_{\mu}^{j}=\mathcal{A}_{\mu}^{j}(k)$ $(j=1, \ldots, n-1)$ which satisfy $\mathrm{H}^{0}\left(\Gamma, \overline{\mathcal{A}}_{\mu}^{j}\right)=0$ since $2 j<p$ and $\mathrm{SL}_{2}\left(k^{\prime}\right) \subset \operatorname{Im} \bar{\rho}_{\pi} \subset \mathrm{GL}_{2}\left(k^{\prime}\right)$. 
2.5. Application of Chebotarev density theorem. Let $M=\mathfrak{g l}_{n}(k)$. Let $r=\operatorname{dim}_{k} \mathrm{H}_{\mathcal{L}_{\bar{\emptyset}}^{\perp}}\left(\Gamma_{\mathbb{Q}}, M^{*}\right)$. For $q$ prime to $N p$ splitting in $K$, let $X^{2}-\bar{a}_{\pi, q} X+q^{a+1}=\left(X-\bar{\alpha}_{q}\right)\left(X-\bar{\beta}_{q}\right)$, where $\bar{\alpha}_{q}, \bar{\beta}_{q} \in k$. We write $\left.\bar{R}\right|_{D_{q}}=\bar{\psi}_{q} \oplus \bar{s}_{q}$ where $\bar{\psi}_{q}=\operatorname{unr}\left(\bar{\alpha}_{q}\right)^{n-1}$ and $\bar{s}_{q}$ is the unramified $D_{q}$-module given by the sum of all eigenspaces corresponding to the other eigenvalues $\bar{\alpha}_{q}^{n-i} \bar{\beta}_{q}^{i}, i \neq 0$. We assume that $\left(\bar{\alpha}_{q} / \bar{\beta}_{q}\right)^{c_{n-1}} \not \equiv 1\left(\bmod \mathfrak{m}_{E}\right)$ so that the eigenvalues $\bar{\alpha}_{q}^{n-i} \bar{\beta}_{q}^{i}, i=0, \ldots, n-1$ are mutually distinct. Therefore $\bar{s}_{q}$ does not contain $\bar{\psi}_{q}$ as $D_{q}$-submodule. Given a finite set of primes $q$ prime to $N p$, split in $K$ as above and such that $q \equiv 1(\bmod p)$, let us define

$$
L_{Q, q}=\mathrm{H}^{1}\left(D_{q} / I_{q}, \operatorname{Ad} \bar{s}_{q}\right) \oplus \mathrm{H}^{1}\left(D_{q}, \operatorname{Ad} \bar{\psi}_{q}\right)
$$

We notice as in [CHT08, Section 2.4.6] the obvious

Lemma 2.11. We have $\operatorname{dim}_{k} L_{Q, q}-h_{q}^{0}(M)=\operatorname{dim}_{k} \mathrm{H}^{1}\left(I_{q}, \operatorname{Ad} \bar{\psi}_{q}\right)^{D_{q}}=1$.

Using $\mathcal{L}_{Q}=\left(L_{Q, v}\right)_{v}$ with $L_{Q, v}$ as before for $v \notin Q$, and the definition above for $v \in Q$, we define $\mathrm{H}_{\mathcal{L}_{Q}}^{1}\left(\Gamma_{\mathbb{Q}}, M\right)$ and $\mathrm{H}_{\mathcal{L}_{Q}^{\perp}}^{1}\left(\Gamma_{\mathbb{Q}}, M^{*}\right)$. Note that, as in [CHT08, Prop.2.4.9], we have a short exact sequence

$$
0 \rightarrow \mathrm{H}_{\mathcal{L}_{Q}^{\perp}}^{1}\left(\Gamma_{Q}, M^{*}\right) \rightarrow \mathrm{H}_{\mathcal{L}^{\perp}}^{1}\left(\Gamma_{Q}, M^{*}\right) \rightarrow \bigoplus_{q \in Q} \mathrm{H}^{1}\left(D_{q} / I_{q}, \operatorname{Ad} \bar{\psi}_{q}(1)\right)
$$

given by the maps $\omega_{q}:[c] \mapsto\left[c_{q}\right]$ for $q \in Q$, where, for any $\sigma \in D_{q}$

$$
c_{q}(\sigma)=p r_{\bar{\psi}_{q}} \circ c(\sigma) \circ i_{\bar{\psi}_{q}}
$$

where $i_{\bar{\psi}_{q}}$ is the inclusion of the $\bar{\psi}_{q}$-line and $p r_{\bar{\psi}_{q}}$ is the projection onto this line parallely to $\bar{s}_{q}$.

Moreover, each term of the right hand side sum is one-dimensional.

Theorem 2.12. For any $m \geq 1$ there exists a set $Q_{m}$ of primes $q$ splitting in $K$, say, $(q)=\mathfrak{q q}^{c}$, and relatively prime to $N p$, such that

- $\sharp Q_{m}=r$

- for any $q \in Q_{m}$, one has $q \equiv 1\left(\bmod p^{m}\right)$

- $\mathrm{H}_{\mathcal{L}_{Q_{m}}^{\perp}}^{1}\left(\Gamma_{\mathbb{Q}}, M^{*}\right)=0$

- for any $q \in Q_{m}, \bar{R}\left(\right.$ Frob $\left._{q}\right)$ has distinct eigenvalues in $k$.

Proof. We follow the proof of [CHT08, Proposition 2.5.9]. Let us first assume that we chose primes $q$ which split totally in $K\left(\zeta_{p^{m}}\right)$ and such that $\bar{R}\left(\right.$ Frob $\left._{q}\right)$ has distinct eigenvalues in $k$. The condition $\mathrm{H}_{\mathcal{L}_{Q_{m}}^{\perp}}^{1}\left(\Gamma_{\mathbb{Q}}, M^{*}\right)=0$ is implied by the isomorphism

$$
\mathrm{H}_{\mathcal{L}_{\emptyset}}^{1}\left(\Gamma_{\mathbb{Q}}, M^{*}\right) \cong \bigoplus_{q \in Q_{m}} \mathrm{H}^{1}\left(D_{q} / I_{q}, \operatorname{Ad} \bar{\psi}_{q}\right)
$$

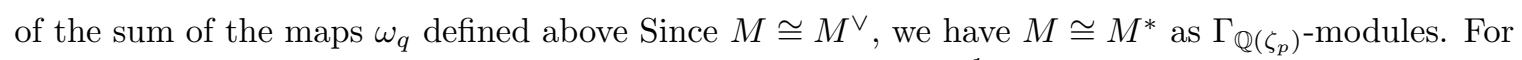
this, it is enough to show that for each non-zero class $[c] \in \mathrm{H}_{\mathcal{L}_{\emptyset}}^{1}\left(\Gamma_{\mathbb{Q}}, M^{*}\right)$ there is a prime $q$ such that $\omega_{q}([c]) \in \mathrm{H}^{1}\left(D_{q} / I_{q}, \operatorname{Ad} \bar{\psi}_{q}\right)$ is non-zero.

By Chebotarev density theorem, it is enough to find for each non zero class $[c] \in \mathrm{H}_{\mathcal{L}_{\emptyset}}^{1}\left(\Gamma_{\mathbb{Q}}, M^{*}\right)$ an element $\sigma \in \Gamma_{\mathbb{Q}}$ such that $\left.\sigma\right|_{K\left(\zeta_{p} m\right)}=1, \bar{R}(\sigma)$ admits an eigenvalue $\gamma$ with multiplicity 1 , and $\operatorname{pr}_{\bar{\gamma}} \circ c(\sigma) \circ i_{\bar{\gamma}} \neq 0$ where $i_{\bar{\gamma}}$ is the injection of the $\bar{\gamma}$-eigenspace of $\bar{R}(\sigma)$ into the space of $\bar{R}$ and $\operatorname{pr}_{\bar{\gamma}}$ the projection to this eigenspace.

Let $F_{m}$ be the extension of $K\left(\zeta_{p^{m}}\right)$ cut out by $\operatorname{Ad} \bar{R}$, that is, the field fixed by the kernel of $\left.\operatorname{Ad} \bar{R}\right|_{\Gamma_{F_{m}}}$. Let us show that $c\left(\Gamma_{F_{m}}\right) \neq 0$. By the inflation-restriction exact sequence

$$
\mathrm{H}^{1}\left(\operatorname{Gal}\left(F_{m} / \mathbb{Q}\right), \operatorname{Ad} \bar{R}\right) \rightarrow \mathrm{H}^{1}\left(\Gamma_{\mathbb{Q}}, \operatorname{Ad} \bar{R}\right) \rightarrow \operatorname{Hom}\left(\Gamma_{F_{m}}, \operatorname{Ad} \bar{R}\right)
$$

it suffices to see that $\mathrm{H}^{1}\left(\operatorname{Gal}\left(F_{m} / \mathbb{Q}\right), \operatorname{Ad} \bar{R}\right)=0$. Consider the inflation-restriction exact sequence

$$
0 \rightarrow \mathrm{H}^{1}\left(\operatorname{Gal}\left(F_{0} / \mathbb{Q}\right), \operatorname{Ad} \bar{R}^{\operatorname{Gal}\left(F_{m} / F_{0}\right)}\right) \rightarrow \mathrm{H}^{1}\left(\operatorname{Gal}\left(F_{m} / \mathbb{Q}\right), \operatorname{Ad} \bar{R}\right) \rightarrow \mathrm{H}^{1}\left(\operatorname{Gal}\left(F_{m} / F_{0}\right), \operatorname{Ad} \bar{R}\right)^{\Gamma_{\mathbb{Q}}}
$$

Since $F_{1} / F_{0}$ is of degree prime to $p$, we have

$$
\mathrm{H}^{1}\left(\operatorname{Gal}\left(F_{m} / F_{0}\right), \operatorname{Ad} \bar{R}\right)^{\Gamma_{\mathbb{Q}}}=\operatorname{Hom}\left(\operatorname{Gal}\left(F_{m} / F_{1}\right), \operatorname{Ad} \bar{R}^{\Gamma_{\mathbb{Q}}}\right)
$$


but $\operatorname{Ad} \bar{R}^{\Gamma_{Q}}=0$ (by bigness), hence the right hand side vanishes. Similarly, $\operatorname{Gal}\left(F_{m} / F_{0}\right)$ acts trivially on $V$ and $\mathrm{H}^{1}\left(\operatorname{Gal}\left(F_{0} / \mathbb{Q}\right), \operatorname{Ad} \bar{R}\right)$ vanishes by bigness (see [CHT08, Cor. 2.5.4]).

Now, we consider $c\left(\Gamma_{\mathbb{Q}\left(\zeta_{p^{m}}\right)}\right)$ as a $\operatorname{Gal}\left(F_{m} / \mathbb{Q}\left(\zeta_{p^{m}}\right)\right)$-submodule of $\operatorname{Ad} \bar{R}$. Note that $\operatorname{Gal}\left(F_{m} / \mathbb{Q}\left(\zeta_{p^{m}}\right)\right)$ contains $\mathrm{PSL}_{2}\left(k^{\prime}\right)$ hence there exists $g \in \operatorname{Gal}\left(F_{m} / \mathbb{Q}\left(\zeta_{p^{m}}\right)\right)$ of order not dividing $p$ fixing a non zero element of $c\left(\Gamma_{\mathbb{Q}\left(\zeta_{p} m\right)}\right)$; we can even assume that $g$ acting on $\operatorname{Ad} \bar{R}$ has distinct eigenvalues in $k$, again by bigness of $\bar{\rho}_{\pi}$, provided that $p-1$ does not divide $c_{n-1}$. Let $\sigma_{0} \in \Gamma_{\mathbb{Q}\left(\zeta_{p} m\right)}$ lifting $g$ and let $\sigma=\tau \sigma_{0}$ where $\tau \in \Gamma_{F_{m}}$ is such that $c(\sigma)=c(\tau)+c\left(\sigma_{0}\right) \notin\left(\sigma_{0}-1\right) V$. Such a $\tau$ exists because $c\left(\Gamma_{\mathbb{Q}\left(\zeta_{p} m\right)}\right) \not \subset(g-1) \operatorname{Ad} \bar{R}$. The corresponding element $\sigma$ satisfies the desired conditions for some $\bar{\gamma} \in k^{\times}$.

For $q \in Q_{m}$, let $X^{2}-\bar{a}_{\pi, q} X+q^{a+1}=\left(X-\bar{\alpha}_{q}\right)\left(X-\bar{\beta}_{q}\right)$, where $\bar{\alpha}_{q}, \bar{\beta}_{q} \in k$. We write $\left.\bar{R}\right|_{D_{q}}=\bar{\psi}_{q} \oplus \bar{s}_{q}$ where $\bar{\psi}_{q}=\operatorname{unr}\left(\bar{\alpha}_{q}\right)^{n-1}$ and $\bar{s}_{q}$ is the unramified $D_{q}$-module given by the sum of all eigenspaces corresponding to the other eigenvalues $\bar{\alpha}_{q}^{n-i} \bar{\beta}_{q}^{i}, i \neq 0$. Note that $\bar{s}_{q}$ does not contain $\bar{\psi}_{q}$ as $D_{q^{-}}$ submodule. We define

$$
L_{Q_{m}, q}=\mathrm{H}^{1}\left(D_{q} / I_{q}, \operatorname{Ad} \bar{s}_{q}\right) \oplus \mathrm{H}^{1}\left(D_{q}, \operatorname{Ad} \bar{\psi}_{q}\right)
$$

We notice as in [CHT08, Section 2.4.6] the obvious

Lemma 2.13. We have $\operatorname{dim}_{k} L_{Q_{m}, q}-h_{q}^{0}(M)=\operatorname{dim}_{k} \mathrm{H}^{1}\left(I_{q}, \operatorname{Ad} \bar{\psi}_{q}\right)^{D_{q}}=1$.

Using $\mathcal{L}_{Q_{m}}=\left(L_{Q_{m}, v}\right)_{v}$ with $L_{Q_{m}, v}$ as before for $v \notin Q_{m}$, and the definition above for $v \in Q_{m}$, we define $\mathrm{H}_{\mathcal{L}_{Q_{m}}}^{1}\left(\Gamma_{Q}, M\right)$ and $\mathrm{H}_{\mathcal{L}_{Q_{m}}^{\perp}}^{1}\left(\Gamma_{Q}, M^{*}\right)$.

Corollary 2.14. For any set $Q_{m}$ as above, one has $\operatorname{dim}_{k} \mathrm{H}_{\mathcal{L}_{Q_{m}}}^{1}\left(\Gamma_{Q}, M\right) \leq \sharp Q_{m}=r$.

2.6. Construction of a Taylor-Wiles system. Recall we fixed in Definition 2.1 a level subgroup $U \subset G(\widehat{\mathbb{Z}})$ of level $N$. Let $Q$ be a finite set of primes $q$ splitting in $K$ such that $q \equiv 1(\bmod p)$ and $\left(\alpha_{q} / \beta_{q}\right)^{c_{n-1}} \not \equiv 1\left(\bmod \mathfrak{m}_{E}\right)$. Let $\Delta_{q}$ be the $p$-Sylow of $(\mathbb{Z} / q \mathbb{Z})^{\times}$. We write $(\mathbb{Z} / q \mathbb{Z})^{\times}=\Delta_{q} \times \Delta_{q}^{p}$. For each $q \in Q, q=\mathfrak{q q} \mathfrak{q}^{c}$, we fix an isomorphism $i_{\mathfrak{q}}: U_{q} \cong \mathrm{GL}_{n}\left(\mathcal{O}_{\mathfrak{q}}\right)$; we identify $\mathcal{O}_{\mathfrak{q}} / \mathfrak{q}=\mathbb{Z} / q \mathbb{Z}$. Let

$$
U_{q}^{\prime}=\left\{g \in U_{q} ; i_{\mathfrak{q}}(g) \equiv\left(\begin{array}{cc}
g_{n-1} & * \\
0 & \delta
\end{array}\right) \quad(\bmod q) \quad \delta \in \Delta_{q}^{p}, g_{n-1} \in \mathrm{GL}_{n-1}\left(\mathcal{O}_{\mathfrak{q}}\right)\right\}
$$

We also write $U_{\mathfrak{q}}^{\prime}=i_{\mathfrak{q}}\left(U_{q}^{\prime}\right)$. We also consider the parahoric group

$$
U_{q, 0}=\left\{g \in U_{q} ; i_{q}(g) \equiv\left(\begin{array}{cc}
g_{n-1} & * \\
0 & \delta
\end{array}\right) \quad(\bmod q) \quad \delta \in(\mathbb{Z} / q \mathbb{Z})^{\times}, g_{n-1} \in \mathrm{GL}_{n-1}\left(\mathcal{O}_{\mathfrak{q}}\right)\right\}
$$

associated to the maximal parabolic subgroup $P \subset \mathrm{GL}_{n}$ fixing the line $<e_{n}>$. Note that $U_{q, 0} / U_{q}^{\prime} \cong$ $\Delta_{q}$. Let $U_{Q}^{\prime}=\prod_{q \in Q} U_{q}^{\prime} \times U^{Q}, U_{Q, 0}=\prod_{q \in Q} U_{q, 0} \times U^{Q}$ and $\Delta_{Q}=\prod_{q \in Q} \Delta_{q}$. Note that $U_{Q, 0} / U_{Q}^{\prime}=$ $\Delta_{Q}$. Let $h_{n-1, Q}$, resp. $\widetilde{h}_{n-1, Q}$, be the (cuspidal) Hida Hecke algebra of auxiliary level group $U_{Q}^{\prime}$ excluding the Hecke operators at $N Q$, resp.including the Atkin-Lehner Hecke operators $U_{q, i}$ at $q \in Q$. This is naturally an $\mathcal{O}\left[\Delta_{Q}\right]$-algebra. We denote by $\mathfrak{a}_{Q}=\left([\delta]-1, \delta \in \Delta_{Q}\right)$ the augmentation ideal of $\mathcal{O}\left[\Delta_{Q}\right]$

If we put $U_{Q}^{b, c}=U_{Q}^{\prime} \cap U^{b, c}$, we note that these algebras both act faithfully on $e \cdot \mathbb{S}_{Q}(E / \mathcal{O})=$ $\lim _{c} e \cdot S_{\lambda}\left(U_{Q}^{c, c} ; E / \mathcal{O}\right)$ (where $\lambda$ is an arbitrary dominant weight, for instance $\lambda=0$ ). By using the

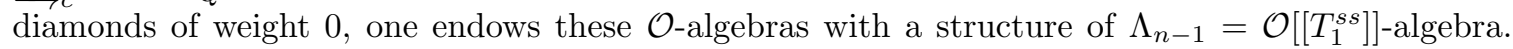
We have a morphism $h_{n-1, Q} \rightarrow h_{n-1}$ which factors through $h_{n-1, Q} \rightarrow h_{n-1, Q} / \mathfrak{a}_{Q} h_{n-1, Q}$.

For any fixed dominant weight $\lambda^{\prime}$ congruent to $((n-1) a,(n-2) a, \ldots, a, 0)$, we also consider the Hecke algebras $e \cdot h_{\lambda^{\prime}}\left(U_{Q}^{0,1}, \mathcal{O}\right)$, resp. $e \cdot \widetilde{h}_{\lambda^{\prime}}\left(U_{Q}^{0,1}, \mathcal{O}\right)$ of weight $\lambda^{\prime}$. Recall that

$$
h_{n-1, Q} / P_{\lambda^{\prime}} h_{n-1, Q} \rightarrow e \cdot h_{\lambda^{\prime}}\left(U_{Q}^{0,1}, \mathcal{O}\right)
$$

is a surjection with nilpotent kernel. Let $\mathfrak{m}_{Q}$ be the maximal ideal of $h_{n-1, Q}$ associated to the residual representation $\bar{R}$. Let $\mathbf{T}_{n-1, Q}=\left(h_{n-1, Q}\right)_{\mathfrak{m}_{Q}}$ resp. $\mathbf{T}_{\lambda^{\prime}, Q}=e \cdot h_{\lambda^{\prime}}\left(U_{Q}^{0,1}, \mathcal{O}\right)_{\mathfrak{m}_{Q}}$ be the corresponding localization-completion. We denote by $R_{Q}^{h}: \Gamma_{\mathbb{Q}} \rightarrow \mathcal{G}_{n}\left(\mathbf{T}_{n-1, Q}\right)$ the lifting of $\bar{R}$ constructed as $R^{h}=$ $R_{\emptyset}^{h}$ over $\mathbf{T}_{n-1}^{u}=\mathbf{T}_{n-1, \emptyset}$. Similarly for $R_{\lambda^{\prime}, Q}^{h}: \Gamma_{\mathbb{Q}} \rightarrow \mathcal{G}_{n}\left(\mathbf{T}_{\lambda^{\prime}, Q}\right)$. 
For $q \in Q$ and for $\beta \in K_{\mathfrak{q}}^{\times}$, we define compatible Frobenius Hecke operators

$$
{ }_{q} V_{\beta}^{c, c}=i_{\mathfrak{q}}^{-1}\left(U_{\mathfrak{q}}^{\prime}\left(\begin{array}{cc}
1_{n-1} & 0 \\
0 & \beta
\end{array}\right) U_{\mathfrak{q}}^{\prime}\right) \times\left(U_{Q}^{c, c}\right)^{q}
$$

It defines an endomorphism of $S_{0}\left(U_{Q}^{c, c}, \mathcal{O}\right)_{\mathfrak{m}_{Q}}$. We fix a lifting $\phi_{q}$ of the geometric Frobenius to $\overline{\mathbb{Q}}_{q}$ given by the Artin symbol $\left[q, \mathbb{Q}_{q}\right]$ on $\mathbb{Q}_{q}^{a b}$. Let $\mathbf{T}_{n-1, Q}^{c, c}=e \cdot h_{0}\left(U_{Q}^{c, c} ; \mathcal{O}\right)_{\mathfrak{m}_{Q}}$ and Let $R_{Q}^{c, c}: \Gamma_{\mathbb{Q}} \rightarrow$ $\mathcal{G}_{n}\left(\mathbf{T}_{n-1, Q}^{c, c}\right)$ be the push-forward of $R_{Q}^{h}$ via the surjective homomorphism $\mathbf{T}_{n-1, Q} \rightarrow \mathbf{T}_{n-1, Q}^{c, c}$. Let $A_{q}^{c, c}$ be the unique root of Char $R_{Q}^{c, c}\left(\phi_{q}\right)$ in $\mathbf{T}_{n-1, Q}$ lifting the root $\alpha_{q}^{n-1} \in k^{\times}$of Char $\bar{R}\left(\phi_{q}\right)$. By Hensel's lemma, for any $c \geq 1$ we have factorizations in $\mathbf{T}_{n-1}^{c, c}$ :

$$
\text { Char } R^{c, c}(X)=\left(X-A_{q}^{c, c}\right) Q_{q}^{c, c}(X) \text {. }
$$

Let $Y_{Q}^{c, c}=\prod_{q \in Q} Q_{q}^{c, c}\left({ }_{q} V_{q}^{c, c}\right) e S_{0}\left(U_{Q}^{c, c}, \mathcal{O}\right)_{\mathfrak{m}_{Q}} ;$ it is the largest $e \cdot h_{0}\left(U_{Q}^{c, c} ; \mathcal{O}\right)_{\mathfrak{m}_{Q}}\left[\left({ }_{q} V_{q}\right)_{q \in Q}\right]$-submodule of $S_{0}\left(U_{Q}^{c, c}, \mathcal{O}\right)_{\mathfrak{m}_{Q}}$ on which for any $q \in Q,{ }_{q} V_{q}^{c, c}-A_{q}^{c, c}$ is topologically nilpotent. Let $\mathbf{T}_{n-1}\left(Y_{Q}^{c, c}\right)$ be the image of $e \cdot h_{0}\left(U_{Q}^{c, c} ; \mathcal{O}\right)_{\mathfrak{m}_{Q}}$ in $\operatorname{End}_{\mathcal{O}}\left(Y_{Q}^{c, c}\right)$. We denote by $R_{Y_{Q}^{c, c}}$ be the image of the representation $R^{h}$ by the projection $\mathbf{T}_{n-1}^{u} \rightarrow \mathbf{T}_{n-1}\left(Y_{Q}^{c, c}\right)$. Recall that by [CHT08, Prop.3.4.4, 8] (here our level groups $U_{Q}^{, c, c}$ play the role of the group $U$ there).

Proposition 2.15. For any $c \geq 1$,

- for any $\beta \in K_{\mathfrak{q}}^{\times} \cap \mathcal{O}_{\mathfrak{q}}$, we have ${ }_{q} V_{\beta}^{c, c} \in \mathbf{T}_{n-1}\left(Y_{Q}^{c, c}\right)$, and $X-{ }_{q} V_{q}^{c, c}$ divides Char $R^{c, c}\left(\phi_{q}\right)$ in $\mathbf{T}_{n-1}\left(Y_{Q}^{c, c}\right)[X]$.

- The map given by ${ }_{q} V^{c, c}\left(\left[\beta, \mathbb{Q}_{q}\right]\right)={ }_{q} V_{\beta}^{c, c}$ for $\beta \in K_{\mathfrak{q}}^{\times} \cap \mathcal{O}_{\mathfrak{q}}$ extends into a continuous character ${ }_{q} V^{c, c}: D_{q} \rightarrow\left(\mathbf{T}_{n-1}\left(Y_{Q}^{c, c}\right)\right)^{\times}$and we have

$$
\left.R_{Y}^{c, c}\right|_{D_{q}}=s_{q} \oplus{ }_{q} V^{c, c}
$$

where $s_{q}$ in unramified of rank $n-1$.

Proof. We refer to [CHT08, Prop.3.4.4, 8] for the details; we simply mention that the proof relies on [CHT08, Lemma 3.1.5] which analyzes the $q$-component of a cuspidal representation occuring in $\mathbf{T}_{n-1}\left(Y_{Q}^{c, c}\right)$; the possibility of a partial Steinberg component is excluded by the condition $q \equiv 1$ $(\bmod p)$.

Actually, by Hensel's lemma one can even define a unique root $A_{q} \in \mathbf{T}_{n-1, Q}$ of Char $R^{h}\left(\phi_{q}\right)$ congruent to $\alpha_{q}^{n-1}$ modulo the maximal ideal such that

$$
\text { Char } R^{h}(X)=\left(X-A_{q}^{c, c}\right) Q_{q}(X)
$$

with $Q_{q}(X) \in \mathbf{T}_{n-1, Q}[X]$ and $Q_{q}\left(A_{q}\right) \in \mathbf{T}_{n-1, Q}^{\times}$. For any $c \geq 1, A_{q}$ interpolates the $A_{q}^{c, c}$ via the morphisms $\mathbf{T}_{n-1, Q} \rightarrow \mathbf{T}_{n-1, Q}^{c, c}$. For any $\beta \in K_{\mathfrak{q}}^{\times} \cap \mathcal{O}_{\mathfrak{q}}$, the operators ${ }_{q} V_{\beta}^{c, c}$ are compatible when $c \geq 1$ varies; they give rise to an element ${ }_{q} V_{\beta} \in \mathbf{T}_{n-1, Q}$ and to a continuous homomorphism

$$
D_{q} \rightarrow \mathbf{T}_{n-1, Q}^{\times}
$$

One can then define a subspace $\mathbb{Y}_{Q} \subset \mathbb{S}_{Q}(E / \mathcal{O})_{\mathfrak{m}_{Q}}$ by $\mathbb{Y}_{Q}=\prod_{q \in Q} Q_{q}\left(V_{q}\right) \mathbb{S}_{Q}(E / \mathcal{O})_{\mathfrak{m}_{Q}}$ such that for any $c \geq 1$, one has $\mathbb{Y}_{Q}^{T_{c}^{s s}}=Y_{Q}^{c, c} \otimes \mathbb{Q}_{p} / \mathbb{Z}_{p}$ via the identification

$$
\mathbb{S}_{Q}^{T_{c}^{s s}}=e \cdot S_{0}\left(U^{c, c} ; E / \mathcal{O}\right) .
$$

We conclude from 2.15 that for any $q \in Q$,

$$
\left.R_{Q}^{h}\right|_{D_{q}}=s_{q} \oplus_{q} V
$$

where $s_{q}$ is unramified and ${ }_{q} V\left(\left[q, \mathbb{Q}_{q}\right]\right)=A_{q}$.

Let $\mathcal{D}_{Q}$ be the deformation subfunctor of $\mathcal{D}$ imposing that for any $q \in Q$, the liftings $r \in \mathcal{D}_{Q}(A)$ of $\bar{R}$ when restricted to $D_{q}$ are of the form

$$
s_{q} \oplus A\left(\psi_{q}\right)
$$


where $s_{q}$ is unramified of rank $n-1$ and lifts $\bar{s}_{q}$, and ${ }_{q} V_{q}: D_{q} \rightarrow A^{\times}$is such that $\psi_{q}\left(\left[q, \mathbb{Q}_{q}\right]\right) \equiv \alpha_{q}^{n-1}$ $\left(\bmod \mathfrak{m}_{A}\right)$. Let $R_{n-1, Q}$ be the universal deformation ring of $\mathcal{D}_{Q}$. It is endowed with a universal lifting

and characters $\psi_{q}^{\text {univ }}: D_{q} \rightarrow\left(R_{Q}^{\text {univ }}\right)^{\times}$such that

$$
R_{Q}^{u n i v}: \Gamma_{Q} \rightarrow \mathcal{G}_{n}\left(R_{Q}^{u n i v}\right)
$$

$$
\left.R_{Q}^{u n i v}\right|_{D_{q}}=s_{q}^{\text {univ }} \oplus \psi_{q}^{\text {univ }} .
$$

where $s_{q}^{\text {univ }}$ is unramified and lifts $\bar{s}_{q}$, and $\psi_{q}^{\text {univ }}\left(\left[q, \mathbb{Q}_{q}\right]\right)$ lifts the root $\bar{\alpha}_{q}^{n-1} \in k^{\times}$of $\bar{R}\left(\operatorname{Frob}_{q}\right)$. Similarly, we have

such that for any $q \in Q$,

$$
R_{\lambda^{\prime}, Q}^{h}: \Gamma_{\mathbb{Q}} \rightarrow \mathcal{G}_{n}\left(\mathbf{T}_{\lambda^{\prime}, Q}\right)
$$

$$
\left.R_{\lambda^{\prime}, Q}^{h}\right|_{D_{q}}=s_{\lambda^{\prime}, q}^{h} \oplus \psi_{\lambda^{\prime}, q}^{h}
$$

where $\psi_{\lambda^{\prime}, q}^{h}={ }_{q} V_{q}^{0,1}$. By Proposition 2.15, these automorphic liftings give rise to surjective ring homomorphisms

$$
R_{n-1, Q} \rightarrow \mathbf{T}_{n-1, Q}\left(\mathbb{Y}_{Q}\right) \text { and } R_{\lambda^{\prime}, Q} \rightarrow \mathbf{T}_{\lambda^{\prime}, Q}\left(\mathbb{Y}_{Q}\right)
$$

sending $R_{Q}^{\text {univ }}$ to $R_{Q}^{h}$ and $\psi_{q}^{\text {univ }}$ to ${ }_{q} V_{q}$, resp. $R_{\lambda^{\prime}, Q}^{\text {univ }}$ to $R_{\lambda^{\prime}, Q}^{h}$ and $\psi_{\lambda^{\prime}, q}^{\text {univ }}$ to ${ }_{q} V_{q}^{0,1}$. Let $\mathfrak{m}_{R_{n-1, Q}}$ resp. $\mathfrak{m}_{R_{\lambda^{\prime}, Q}}$ be the maximal ideal of $R_{\lambda^{\prime}, Q}$. Note that we have canonically

$$
\mathfrak{m}_{R_{n-1, Q}} /\left(\mathfrak{m}_{\mathcal{O}}+\mathfrak{m}_{R_{n-1, Q}}^{2}\right)=\mathfrak{m}_{R_{\lambda^{\prime}, Q}} /\left(\mathfrak{m}_{\mathcal{O}}+\mathfrak{m}_{R_{\lambda^{\prime}, Q}}^{2}\right)
$$

and that the $k$-dual of this space is canonically isomorphic to $\mathrm{H}_{\mathcal{L}_{Q}}^{1}\left(\Gamma_{\mathbb{Q}}, M\right)$. Moreover, it follows from 2.14 that

Corollary 2.16. For any set $Q_{m}$ as above and for $r=\operatorname{dim}_{k} \mathrm{H}_{\mathcal{L}_{\emptyset}}^{1}\left(\Gamma_{\mathbb{Q}}, M\right)$, one has

$$
\operatorname{dim}_{k} \mathfrak{m}_{R_{n-1, Q_{m}}} /\left(\mathfrak{m}_{\mathcal{O}}+\mathfrak{m}_{R_{n-1, Q_{m}}}^{2}\right)=\operatorname{dim}_{k} \mathrm{H}_{\mathcal{L}_{Q_{m}}}^{1}\left(\Gamma_{\mathbb{Q}}, M\right) \leq \sharp Q_{m}=r
$$

2.7. End of the proof. We first fix a regular dominant weight $\lambda^{\prime}$ congruent modulo $p-1$ to $((n-1) a,(n-2) a, \ldots, a, 0)$. We assume either 1a) or 1b), so that the characters on the diagonal of Symm $\left.^{n-1} \bar{\rho}_{\mu}\right|_{D_{d}}$ are mutually distinct. We consider the diagram of morphisms

$$
\begin{array}{ccc}
R_{\lambda^{\prime}, Q_{m}} & \rightarrow & \mathbf{T}_{\lambda^{\prime}, Q_{m}}\left(\mathbb{Y}_{Q_{m}}\right) \\
\downarrow & & \downarrow \\
R_{\lambda^{\prime}} & \rightarrow & \mathbf{T}_{\lambda^{\prime}}\left(U^{0,1}, \mathcal{O}\right)
\end{array}
$$

The first line is $\mathcal{O}\left[\Delta_{Q_{m}}\right]$-linear. Let $\mathbf{M}_{\lambda^{\prime}, Q_{m}}$ be the Pontryagin dual of $\mathbb{Y}_{Q_{m}}$ and $\mathbf{M}_{\lambda^{\prime}, 0, Q_{m}}$ be the Pontryagin dual of the analogue $\mathbb{Y}_{Q_{m}, 0}$ of $\mathbb{Y}_{Q_{m}}$ obtained by replacing the level group $U_{Q_{m}}$ by $U_{Q_{m}, 0}$. Similarly, let $\mathbf{M}_{\lambda^{\prime}, \emptyset}$ be the Pontryagin dual of $e \cdot S_{\lambda^{\prime}}\left(U^{0,1}, E / \mathcal{O}\right)_{\mathfrak{m}}$. We know that $\mathbf{M}_{\lambda^{\prime}, Q_{m}}$ is free of finite rank over $\mathcal{O}\left[\Delta_{Q_{m}}\right]$ and admits a faithful action of $\mathbf{T}_{\lambda^{\prime}, Q_{m}}\left(\mathbb{Y}_{Q_{m}}\right)$. By [Ge10, Lemma 2.2.6], we have

One also knows that

$$
\mathbf{M}_{\lambda^{\prime}, Q_{m}} / \mathfrak{a}_{Q_{m}} \mathbf{M}_{\lambda^{\prime}, Q_{m}} \cong \mathbf{M}_{\lambda^{\prime}, 0, Q_{m}}
$$

$$
R_{\lambda^{\prime}, Q_{m}} / \mathfrak{a}_{Q_{m}} R_{\lambda^{\prime}, Q_{m}} \cong R_{\lambda^{\prime}}
$$

By Cor.2.14, there are surjections in $C N L_{\mathcal{O}}$ :

$$
\mathcal{O}\left[\left[Y_{1}, \ldots, Y_{r}\right]\right] \rightarrow R_{\lambda^{\prime}, Q_{m}}
$$

Let $\Psi_{m}$ : be the composition

$$
\mathcal{O}\left[\left[Y_{1}, \ldots, Y_{r}\right]\right] \rightarrow R_{\lambda^{\prime}, Q_{m}} \rightarrow R_{\lambda^{\prime}}
$$

We also have surjections

$$
\mathcal{O}\left[\left[Z_{1}, \ldots, Z_{r}\right]\right] \rightarrow \mathcal{O}\left[\Delta_{Q_{m}}\right]
$$

whose kernels $\mathfrak{n}_{m}$ satisfy $\bigcap_{m} \mathfrak{n}_{m}=(0)$. We can lift the map

$$
\mathcal{O}\left[\left[Z_{1}, \ldots, Z_{r}\right]\right] \rightarrow \mathcal{O}\left[\Delta_{Q_{m}}\right] \rightarrow R_{\lambda^{\prime}, Q_{m}}
$$

to a map

$$
\Phi_{m}: \mathcal{O}\left[\left[Z_{1}, \ldots, Z_{r}\right]\right] \rightarrow \mathcal{O}\left[\left[Y_{1}, \ldots, Y_{r}\right]\right]
$$


The composition

$$
\Psi_{m} \circ \Phi_{m}: \mathcal{O}\left[\left[Z_{1}, \ldots, Z_{r}\right]\right] \rightarrow R_{\lambda^{\prime}} / \mathfrak{m}_{\mathcal{O}} R_{\lambda^{\prime}}
$$

has kernel $\left(Z_{1}, \ldots, Z_{r}\right)+\mathfrak{m}_{\mathcal{O}}$

On the other hand, it follows from [CHT08, Cor.3.1.5] that we have a Hecke linear isomorphism

$$
\mathbf{M}_{\lambda^{\prime}, Q_{m}, 0} \cong \mathbf{M}_{\lambda^{\prime}, \emptyset}
$$

so that

$$
\mathbf{M}_{\lambda^{\prime}, Q_{m}} / \mathfrak{a}_{Q_{m}} \mathbf{M}_{\lambda^{\prime}, Q_{m}} \cong \mathbf{M}_{\lambda^{\prime}, \emptyset}
$$

One can now apply Diamond-Fujiwara's version of the Taylor-Wiles machine (see Th.2.1 of [Dia97] as at the end of the proof of [CHT08, Theorem 3.5.1] to conclude that the morphism

$$
R_{\lambda^{\prime}} \rightarrow \mathbf{T}_{\lambda^{\prime}}\left(U^{0,1}, \mathcal{O}\right)
$$

is an isomorphism in $C N L_{\mathcal{O}}$, that $M_{\lambda^{\prime}, \emptyset}$ is free over $\mathbf{T}_{\lambda^{\prime}}\left(U^{0,1}, \mathcal{O}\right)$ and that these algebras are local complete intersection.

In order to deduce that $R_{n-1} \rightarrow \mathbf{T}_{n-1}^{u}$ is an isomorphism of $\Lambda_{n-1}$-algebras and that they are local complete intersection, we proceed as in [Ti06, Sect.3.2]. We choose a regular dominant weight $\lambda^{\prime}$ congruent to $((n-1) a, \ldots, a, 0)$ and we consider the diagram

$$
\begin{array}{ccc}
R_{n-1} / P R_{n-1} & \rightarrow & \mathbf{T}_{n-1}^{u} / P \mathbf{T}_{n-1}^{u} \\
\downarrow & & \downarrow \\
R_{\lambda^{\prime}} & \rightarrow & \mathbf{T}_{\lambda^{\prime}}\left(U^{0,1}, \mathcal{O}\right)
\end{array}
$$

where $P=P_{\lambda^{\prime}}$. We know that the bottom line is an isomorphism and that the first column is an isomorphism. It follows that the first line is an isomorphism. and that $\mathbf{T}_{n-1}^{u} / P \mathbf{T}_{n-1}^{u} \cong$ $\mathbf{T}_{\lambda^{\prime}}\left(U^{0,1}, \mathcal{O}\right)$ is contained in $P R_{n-1}$. Moreover, by Hida's control theorem $\mathbf{M}_{n-1} / P \mathbf{M}_{n-1}=\mathbf{M}_{\lambda^{\prime}, \emptyset}$; hence $\mathbf{M}_{n-1} / P \mathbf{M}_{n-1}$ is free over $\mathbf{T}_{n-1}^{u} / P \mathbf{T}_{n-1}^{u}$. This implies by Nakayama's lemma that $\mathbf{M}_{n-1}$ is free over $T_{n-1}^{u}$ (use that $\mathbf{M}_{n-1}$ is free over $\Lambda_{n-1}$ by Hida theory). In particular, $\mathbf{T}_{n-1}^{u}$ is free over $\Lambda_{n-1}$. From this we can deduce by a similar argument that the injectivity of $R_{n-1} / P R_{n-1} \rightarrow$ $\mathbf{T}_{n-1}^{u} / P \mathbf{T}_{n-1}^{u}$ implies the injectivity of $R_{n-1} \rightarrow \mathbf{T}_{n-1}^{u}$. Since $P$ is generated by a regular sequence in $\Lambda_{n-1}$ and since $\mathbf{T}_{n-1}^{u} / P \mathbf{T}_{n-1}^{u}$ is local complete intersection over $\Lambda_{n-1} / P$, the same holds for $\mathbf{T}_{n-1}^{u}$ over $\Lambda_{n-1}$.

\section{Proof of Theorem 1.3}

3.1. The case $j=3$. The proof of Theorem 1.3 makes use of the $\operatorname{Symm}^{3}$ base change from $G L_{2}(\mathbb{Q})$ to $\mathrm{GSp}_{4}(\mathbb{Q})$ as established in $[\mathrm{RS} 07]$. The level of the $\mathrm{Symm}^{3}$ of a newform of squarefree Iwahori level is still squarefree Iwahori [RS07]. Let $h_{2}$ be the Hida Hecke algebra constructed in [TU99] (see also [H02] or [Ti06]). It is a finite torsion-free algebra over $\Lambda_{2}=\mathbb{Z}_{p}\left[\left[X_{1}, X_{2}\right]\right]$. Calculations detailed in [Con16a, Section 3.3] describe the only possible homomorphism

$$
\mathcal{H}\left(\mathrm{GSp}_{4}\right)^{N p} \otimes \mathcal{H}_{p}\left(\mathrm{GSp}_{4}\right)^{I w,-} \rightarrow \mathcal{H}\left(\mathrm{GL}_{2}\right)^{N p} \otimes \mathcal{H}_{p}\left(\mathrm{GL}_{2}\right)^{I w,-}
$$

between our abstract Hecke algebras, deduced from the base change map from $\mathrm{GL}_{2}$ to GSp 4 and compatible with the ordinarity condition. In fact, in [Con16a, Proposition 3.3.5], A. Conti defines eight homomorphisms $\lambda^{N p} \otimes \lambda_{p, i}, i=1, \ldots, 8$ in the context of finite slope case, but only the first is compatible with our ordinarity assumption. It provides a commutative diagram of algebra homomorphisms

$$
\begin{array}{ccc}
h_{2} & \stackrel{\theta}{\rightarrow} & h_{1} \\
\uparrow & & \uparrow \\
\Lambda_{2} & \rightarrow & \Lambda_{1}
\end{array}
$$

the bottom homomorphism is induced on the highest weights of local systems by $n \geq 0 \mapsto(a, b)$ where $a \geq b \geq 0$ are given by $a=2 n$ and $b=n$. Here, $n \geq 0$ corresponds to the irreducible representation $\mathrm{Symm}^{n} \mathrm{St}_{2}$ of highest weight $n$ of $\mathrm{GL}_{2}(\mathbb{Q})$ and $(a, b)$ corresponds to the similar Weyl representation of $G \mathrm{Sp}_{4}(\mathbb{Q})$. For any prime $\ell$ not dividing $N p$, let the universal genus 2 Hecke polynomial at $\ell$

$$
P_{\ell}^{(2)}(X)=X^{4}-T_{\ell} X^{3}+\ell\left(R_{\ell}+\left(1+\ell^{2} S_{\ell}\right)\right) X^{2}-\ell^{3} T_{\ell} S_{\ell} X+\ell^{6} S_{\ell}^{3}
$$


where the coefficients are given by the universal Hecke operators with the notations of Conjecture 2 Section 7 of [TU99]. Then,the homomorphism $\theta$ is defined as follows. For any prime $\ell$ not dividing $N p$, it send the coefficients of $P_{\ell}^{(2)}(X)$ to those of the symmetric cube $\operatorname{Symm}^{3} P_{\ell}^{(1)}=$ $\left(X-U_{\ell}^{3}\right)\left(X-U_{\ell}^{2} V_{\ell}\right)\left(X-U_{\ell} V_{\ell}^{2}\right)\left(X-V_{\ell}^{3}\right)$ of the genus 1 universal Hecke polynomial $P_{\ell}^{(1)}=X^{2}-$ $T_{\ell} X+\ell S_{\ell}=\left(X-U_{\ell}\right)\left(X-V_{\ell}\right)$. For $\ell=p$, let $U_{p, 1}$, resp. $U_{p, 2}$, be the double class of $\operatorname{diag}(p, p, 1,1)$, resp. $\operatorname{diag}\left(p, p^{2}, p, 1\right)$, for the Iwahori subbgroup of $\operatorname{GSp}_{4}\left(\mathbb{Z}_{p}\right)$. Then by standard calculations (see [Con16a, Proposition 3.3.5 and Corollary 3.3.9], we see that $\theta$ sends $U_{p, 1}$ to $U_{p}^{3}$. and $U_{p, 2}$ to $U_{p}^{4}$

Let $\mathbf{T}_{2}$ be the localization of $h_{2}$ at the maximal ideal associated to $\operatorname{Symm}^{3} \bar{\rho}_{\mu}$; the morphism $\theta$ factors through $\mathbf{T}_{2} \rightarrow \mathbf{T}_{1}$. Let $\lambda=\mu \circ \theta$. Let $\widetilde{\mathbf{T}}_{2}=\mathbf{T}_{2} \otimes_{\Lambda_{2}} \widetilde{A}_{1}$ Let us still denote by the same letters the homomorphisms obtained by extensions of scalars to $\widetilde{A}_{1}$ :

$$
\widetilde{\mathbf{T}}_{2} \stackrel{\theta}{\rightarrow} \widetilde{\mathbf{T}}_{1} \stackrel{\mu}{\rightarrow} \widetilde{A}_{1}
$$

and their composition $\lambda=\mu \circ \theta$. Let us recall Theorem 4.2 of [Pi12b] (especially, in the context of deformations of a residual representation $\operatorname{Symm}^{3} \bar{\rho}_{\mu}$, treated in section 5.8.2 of this paper). Let $R_{2}$ be the minimal $p$-ordinary universal deformation ring of $\operatorname{Symm}^{3} \bar{\rho}_{\mu}$.

Theorem 3.1. Assuming the assumptions $(*)$ and that either $\alpha^{12} \not \equiv 1\left(\bmod \mathfrak{m}_{A_{1}}\right)$ or $3(a+1)<p-1$, we have $R_{2}=\mathbf{T}_{2}$ and $\mathbf{T}_{2}$ is local complete intersection over $\Lambda_{2} ;$ moreover it is finite flat over $\Lambda_{2}$.

Note that the auxiliary level of $h_{2}$ is Iwahori of the same squarefree level $N$ as $h_{1}$.

Note that the theorem implies that $\mathbf{T}_{2} \rightarrow \mathbf{T}_{1}$ is surjective.

The $\Lambda_{1}$-algebra $\widetilde{\mathbf{T}}_{2}$ is reduced and we have quasi-splittings of $\mu, \theta$ and $\lambda$ :

$$
\begin{gathered}
(1) \quad \widetilde{\mathbf{T}}_{1} \otimes_{\widetilde{A}_{1}} \mathcal{K}_{1} \cong \mathcal{K}_{1} \times \widetilde{\mathbf{T}}_{\mu, \mathcal{K}_{1}}^{\prime} \\
(2) \quad \widetilde{\mathbf{T}}_{2} \otimes_{\widetilde{A}_{1}} \mathcal{K}_{1} \cong\left(\widetilde{\mathbf{T}}_{1} \otimes_{\widetilde{A}_{1}} \mathcal{K}_{1}\right) \times \widetilde{\mathbf{T}}_{\theta, \mathcal{K}_{1}}^{\prime} \\
\text { (3) } \widetilde{\mathbf{T}}_{2} \otimes_{\widetilde{A}_{1}} \mathcal{K}_{1} \cong \mathcal{K}_{1} \times \widetilde{\mathbf{T}}_{\lambda, \mathcal{K}_{1}}^{\prime}
\end{gathered}
$$

Let $\widetilde{\mathbf{T}}_{\theta}^{\prime}$, resp. $\widetilde{\mathbf{T}}_{\lambda}^{\prime}$, be the image of $\widetilde{\mathbf{T}}_{2}$ by the second projection in (2), resp. in (3). Besides the ideal $\mathfrak{c}_{\mu}$ already defined, one can define two other congruence ideals :

$$
\mathfrak{c}_{\theta}=\widetilde{\mathbf{T}}_{2} \cap\left(\widetilde{\mathbf{T}}_{1} \times\left\{0_{\widetilde{\mathbf{T}}_{\theta}^{\prime}}\right\}\right)
$$

and

$$
\mathfrak{c}_{\lambda}=\widetilde{\mathbf{T}}_{2} \cap\left(\widetilde{A}_{1} \times\left\{0_{\widetilde{\mathbf{T}}_{\lambda}^{\prime}}\right\}\right)
$$

Corollary 3.2. Assume (*).

1) Assume either $\alpha^{12} \not \equiv 1\left(\bmod \mathfrak{m}_{A_{1}}\right)$ or $3(a+1)<p-1$, then the ideals $\mathfrak{c}_{\lambda}$, $\mathfrak{c}_{\mu}$ and $\lambda\left(\mathfrak{c}_{\theta}\right)$ are principal and we have the relation

$$
\mathfrak{c}_{\lambda}=\mathfrak{c}_{\mu} \lambda\left(\mathfrak{c}_{\theta}\right)
$$

Proof. We know that $\mathbf{T}_{i}$ is local complete intersection over $\Lambda_{i}(i=1,2)$. By flatness of $\widetilde{A}_{1}$ over $\Lambda_{1}$, it follows that $\widetilde{\mathbf{T}}_{i}(i=1,2)$ are local complete intersection over $\widetilde{A}_{1}$. Thus, statement follows from 8.5 and 8.8 .

Proposition 3.3. 2) If one assumes that $3(a+1)<p-1$, the ideal $\mathfrak{c}_{\lambda}$ is generated by $\operatorname{Char}\left(\operatorname{Sel}_{(}\left(\operatorname{Ad}_{\mathfrak{s p}} \rho_{\mu}\right)\right)$.

Proof. Let $\mathcal{L}$ be the composition of the isomorphism $R_{2} \rightarrow \mathbf{T}_{2}$ with $\lambda: \mathbf{T}_{2} \rightarrow A_{1}, \widetilde{R}_{2}=R_{2} \otimes_{\Lambda_{2}} \widetilde{A}_{1}$ and $\widetilde{\mathcal{L}}: \widetilde{R}_{2} \rightarrow \widetilde{A}_{1}$ the composition of $\mathcal{L} \otimes \operatorname{Id}_{\widetilde{A}_{1}}$ with the multiplication $A_{1} \otimes \widetilde{A}_{1} \rightarrow \widetilde{A}_{1}$. By flatness of $\widetilde{A}_{1}$ over $\Lambda_{1}$, we see that $\widetilde{R}_{2}$ is local complete intersection over $\widetilde{A}_{1}$. We first apply 8.7 to see that the principal ideal $\mathfrak{c}_{\lambda}$ coincides with the reflexive envelope of $\operatorname{Fitt}_{0}\left(C_{1}\left(\widetilde{\mathcal{L}}, \widetilde{A}_{1}\right)\right)$. It remains to see that

$$
C_{1}\left(\widetilde{\mathcal{L}}, \widetilde{A}_{1}\right) \cong \operatorname{Sel}\left(\operatorname{Ad}_{\mathfrak{s p}} \rho_{\mu}\right)
$$

It is only to prove this that we also need to assume $3(a+1)<p-1$. Indeed, let $I=\operatorname{Ker}\left(\Lambda_{2} \rightarrow \Lambda_{1}\right)$. It is a principal ideal, say $I=(\xi)$. The quotient $R_{2}^{\prime}=R_{2} / \xi R_{2}$ is local complete intersection over 
$\Lambda_{1}$ and is the deformation ring of symplectic $N$-minimal ordinary deformations whose Hodge-Tate weights are of the form $(3 h, 2 h, h, 0)$. We have $\Omega_{R_{2} / \Lambda_{2}} \otimes_{\Lambda_{2}} \widetilde{A}_{1}=\Omega_{R_{2}^{\prime} / \Lambda_{1}} \otimes_{\Lambda_{1}} \widetilde{A}_{1}$. By flatness of $\widetilde{A}_{1}$ over $\Lambda_{1}$, we conclude

$$
\Omega_{R_{2} / \Lambda_{2}} \otimes_{\Lambda_{2}} \widetilde{A}_{1}=\Omega_{\widetilde{R}_{2} / \widetilde{A}_{1}} .
$$

Let us now compute the Pontryagin dual of $C_{1}\left(\widetilde{\mathcal{L}}, \widetilde{A}_{1}\right)$ :

$$
\begin{aligned}
C_{1}\left(\widetilde{\mathcal{L}}, \widetilde{A}_{1}\right)^{*} & =\operatorname{Hom}_{R_{2}}\left(\Omega_{\widetilde{R}_{2} / \widetilde{A}_{1}}, \widetilde{A}_{1}^{*}\right)=\operatorname{Hom}_{R_{2}}\left(\Omega_{R_{2} / \Lambda_{2}} \otimes_{\Lambda_{2}} \widetilde{A}_{1}, \widetilde{A}_{1}^{*}\right) \\
& =\operatorname{Hom}_{R_{2}}\left(\Omega_{R_{2} / \Lambda_{2}}, \widetilde{A}_{1}^{*}\right)=\operatorname{Der}_{\Lambda_{2}}\left(R_{2}, \widetilde{A}_{1}^{*}\right)
\end{aligned}
$$

Let $R_{2}\left(\widetilde{A}_{1}^{*}\right)=R_{2} \oplus \epsilon \cdot \widetilde{A}_{1}^{*}$ with $\epsilon^{2}=0$, then

$$
\operatorname{Der}_{\Lambda_{2}}\left(R_{2}, \widetilde{A}_{1}^{*}\right)=\operatorname{Hom}_{\mathcal{L}}\left(R_{2}\left(\widetilde{A}_{1}^{*}\right), \widetilde{A}_{1}^{*}\right)=\left\{\rho \in \mathcal{D}\left(R_{2}\left(\widetilde{A}_{1}^{*}\right)\right) ; \rho(\sigma)=(\mathbf{1}+\epsilon \cdot c(\sigma)) \rho^{\text {univ }}(\sigma)\right\} / \sim .
$$

The map $\rho \mapsto c$ induces an injective map $\Phi$ from this set (which happens to be a group) to the group of cohomology classes $[c] \in \mathrm{H}^{1}\left(\Gamma_{\mathbb{Q}}, \mathfrak{g l}_{n}\left(A_{1}\right) \otimes_{A_{1}} \widetilde{A}_{1}^{*}\right)$. The image of $\Phi$ is contained in the subgroup of the cohomology classes such that for $\ell$ dividing $N, c\left(I_{\ell}\right)=0$ (this is the $N$-minimality condition) and such that $\left.c\right|_{I_{p}}$ takes values, up to conjugation, in $\mathfrak{n}_{n}^{+}\left(A_{1}\right) \otimes_{A_{1}} \widetilde{A}_{1}$ where $\mathfrak{n}_{n}^{+}\left(A_{1}\right)=F i l^{1} \operatorname{Ad}\left(\operatorname{Symm}^{3} \rho_{\mu}\right)$ is the upper nilpotent subgroup of $\mathfrak{g l}_{n}\left(A_{1}\right)=\operatorname{Ad}\left(\operatorname{Symm}^{3} \rho_{\mu}\right)$. This is the min-ord condition. Note that if $\rho$ is upper triangular on $I_{p}$ it is still so on $D_{p}$. The condition

$$
\text { (1b) } 3(a+1)<p-1
$$

has not yet been used. Recall it is equivalent to saying that the characters on the diagonal of Symm $\left.^{3} \bar{\rho}_{\mu}\right|_{I_{p}}$ are mutually distinct. This condition implies the surjectivity of $\Phi$. Indeed, any cocycle $c$ define a unique conjugacy class $[\rho]$ of liftings of $\bar{\rho}$ We need to check that $[\rho]$ defines a deformation in $\mathcal{D}\left(R_{2}\left(\widetilde{A}_{1}^{*}\right)\right)$; it amounts to verifying that the characters defined by $\left.\rho\right|_{D_{p}}$ are in the right order on the full decomposition group, knowing that they are in the right order on the inertia at $p$. This is precisely what condition (1b) imposes.

On the other hand, for $p>3$, we have a decomposition of $\mathbb{Z}_{p}$-representations of $\mathrm{GL}_{2}: \mathrm{Ad}_{\mathfrak{s p}_{4}}=$ $\mathcal{A}^{1} \oplus \mathcal{A}^{3}$. This implies a decomposition of minimal $p$-ordinary Selmer groups

$$
\operatorname{Sel}\left(\operatorname{Ad}_{\mathfrak{s p}} \rho_{\mu}\right)=\operatorname{Sel}\left(\mathcal{A}_{\mu}^{1}\right) \oplus \operatorname{Sel}\left(\mathcal{A}_{\mu}^{3}\right) .
$$

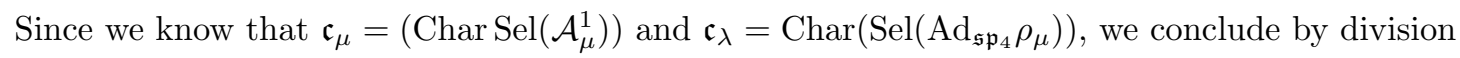

Corollary 3.4. The ideal $\lambda\left(\mathfrak{c}_{\theta}\right)$ is principal generated by $\operatorname{Char}\left(\operatorname{Sel}\left(\mathcal{A}_{\mu}^{3}\right)\right)$.

By definition, the associated primes of $\lambda\left(\mathfrak{c}_{\theta}\right)$ in in $\widetilde{A}_{1}$ are congruence primes between $\operatorname{Symm}^{3}(\mu)$ and Siegel families which are not $\mathrm{Symm}^{3}$ of $G L_{2}(\mathbb{Q})$ families. Because of the Greenberg-Iwasawa conjecture, it is natural to conjecture that the (not yet constructed) $p$-adic $L$ function of $\mathcal{A}_{\mu}^{3}$ generates the ideal $\lambda\left(\mathfrak{c}_{\theta}\right)$, hence controls then congruences of the above type. This understands a decomposition (not only up to algebraic numbers but up to $p$-adic units in a number field) of periods according to the decomposition

$$
L\left(\operatorname{Ad}_{\mathfrak{s p}_{4}}(\mu), s\right)=L\left(\mathcal{A}_{\mu}^{1}, s\right) L\left(\mathcal{A}_{\mu}^{3}, s\right) .
$$

3.2. The case $j=2$. In order to treat the case $j=2$, we assume that the integer $a$ associated to $\mathbf{T}_{1}$ (hence to $\mu$ ) satisfies $3(a+1)<p-1$. We also use another decomposition of $\mathbb{Z}_{p}$-representations of $\mathrm{GL}_{2}$ (valid if $p>3$ ) :

$$
\operatorname{Ad}_{\mathfrak{s l}_{4}}=\mathcal{A}^{1} \oplus \mathcal{A}^{2} \oplus \mathcal{A}^{3}
$$

The general formulas over $\mathbb{Z}_{p}$ are actually

$$
\operatorname{Ad}_{\mathfrak{s} \mathfrak{l}_{m}}=\bigoplus_{i=1}^{m-1} \mathcal{A}^{i}
$$


for any $m \geq 2$, provided $p>m-1$, and

$$
\operatorname{Ad}_{\mathfrak{s p}_{2 m}}=\bigoplus_{j=1}^{m} \mathcal{A}^{2 j-1}
$$

for any $m \geq 1$ provided $p>2 m-1$.

As already mentioned, the $\mathrm{Symm}^{3}$ base change to $G L_{4}(\mathbb{Q})$ is established by Kim. Recall that we fixed a squarefree level $N$ and a prime factor $q_{1}$ thereof. As above, we choose an auxiliary imaginary quadratic field in which $p$ and $q_{1}$ split. We then choose a degree 16 skew field of center $K$ with second kind involution, which ramifies exactly at those two primes. There exists a unitary group $U(4)$ compact at infinity, quasi split at all inert primes. By Arthur and Clozel, automorphic forms can be transfered from $G L_{2}(\mathbb{Q})$ to $U(4)$. In his thesis [Ge10], D. Geraghty defined a Hida Hecke algebra $h_{3}^{u}$ associated to $U(4)$ which is finite torsion free over the Iwasawa algebra $\Lambda_{3}=\mathbb{Z}_{p}\left[\left[X_{1}, X_{2}, X_{3}\right]\right]$. At this stage, it is better to write $h_{2}^{s}$ for the Hecke algebra for symplectic forms previously denoted $h_{2}$, in order to distinguish unitary and symplectic group Hecke algebras. The Symm ${ }^{3}$ base change provides a ring homomorphism $h_{3}^{u} \rightarrow h_{1}$ with a commutative diagram

$$
\begin{array}{ccc}
h_{3}^{u} & \rightarrow & h_{1} \\
\uparrow & & \uparrow \\
\Lambda_{3} & \rightarrow & \Lambda_{1}
\end{array}
$$

The bottom map is induced by $n \mapsto\left(\lambda_{1}, \lambda_{2}, \lambda_{3}\right)$ where $\lambda_{1} \geq \lambda_{2} \geq \lambda_{3} \geq \mathbf{0}$ are given by $\lambda_{1}=3 n$, $\lambda_{2}=2 n$ and $\lambda_{3}=n$. But we need a more precise information about this diagram. For this, we note that the base change from $G S p_{4}$ to $G L_{4}$ has also been established [Mok14], so that there is also a commutative diagram of ring homomorphisms

$$
\begin{array}{ccccccc}
h_{3}^{u} & \stackrel{\theta^{\prime}}{\rightarrow} & h_{2}^{s} & \stackrel{\theta}{\rightarrow} & h_{1} & \stackrel{\mu}{\rightarrow} & A_{1} \\
\uparrow & & \uparrow & & \uparrow & & \uparrow \\
\Lambda_{3} & \rightarrow & \Lambda_{2} & \rightarrow & \Lambda_{1} & = & \Lambda_{1}
\end{array}
$$

where the first bottom arrow is given by $(a, b) \mapsto\left(\lambda_{1}, \lambda_{2}, \lambda_{3}\right)$ where $a \geq b \geq 0$ and $\lambda_{1} \geq \lambda_{2} \geq \lambda_{3} \geq \mathbf{0}$ are given by $\lambda_{1}=a+b, \lambda_{2}=a$ and $\lambda_{3}=b$. Let $\mathbf{T}_{3}^{u}$ be the localization of $h_{3}^{u}$ at the maximal prime corresponding to $\operatorname{Symm}^{3} \bar{\rho}_{\mu}$. The morphism $\theta^{\prime}$ factors through $\mathbf{T}_{3}^{u}$ and is still denoted as $\theta^{\prime}: \mathbf{T}_{3}^{u} \rightarrow \mathbf{T}_{2}^{s}$. Let $\lambda^{\prime}=\lambda \circ \theta^{\prime}$. Let $\widetilde{\mathbf{T}}_{3}^{u}=\mathbf{T}_{3}^{u} \otimes_{\Lambda_{3}} \widetilde{A}_{1}$. We tensorize the morphisms by $\widetilde{A}_{1}$ (without changing the notation) and we get $\widetilde{A}_{1}$-algebra homomorphisms

$$
\widetilde{\mathbf{T}}_{3}^{u} \stackrel{\theta^{\prime}}{\rightarrow} \widetilde{\mathbf{T}}_{2}^{s} \stackrel{\lambda}{\rightarrow} \widetilde{A}_{1}
$$

Let $\mathcal{G}_{4}=\left(\mathrm{GL}_{4} \times \mathrm{GL}_{1}\right) \rtimes\{1, j\}$ where $j(g, \nu) j^{-1}=\left(\nu^{t} g^{-1}, \nu\right)$. Let $R_{3}$ be the minimal $p$-ordinary universal ring of deformations $\rho: G_{\mathbb{Q}} \rightarrow \mathcal{G}_{4}(B)$ of $\operatorname{Symm}^{3} \bar{\rho}_{\mu}: G_{\mathbb{Q}} \rightarrow G L_{4}(k)$. By treating a simpler case than in [Ge10], we prove

Theorem 3.5. Assuming $(*)$ and either $\alpha^{12} \not \equiv 1\left(\bmod \mathfrak{m}_{A_{1}}\right)$ or $3(a+1)<p-1$, we have $R_{3}=\mathbf{T}_{3}^{u}$, and this ring is local complete intersection over $\Lambda_{3}$; in particular it is finite flat over $\Lambda_{3}$.

Again, the $\Lambda_{1}$-algebra $\widetilde{\mathbf{T}}_{3}^{u}$ is reduced and we have quasi-splittings of $(\lambda$ and $) \theta^{\prime}$ and $\lambda^{\prime}$ :

$$
\begin{gathered}
\left(2^{\prime}\right) \quad \widetilde{\mathbf{T}}_{3}^{u} \otimes_{\widetilde{A}_{1}} \mathcal{K}_{1} \cong\left(\widetilde{\mathbf{T}}_{2}^{s} \otimes_{\widetilde{A}_{1}} \mathcal{K}_{1}\right) \times \widetilde{\mathbf{T}}_{\theta^{\prime}, \mathcal{K}_{1}}^{\prime} \\
\left(3^{\prime}\right) \quad \widetilde{\mathbf{T}}_{3}^{u} \otimes_{\widetilde{A}_{1}} \mathcal{K}_{1} \cong \mathcal{K}_{1} \times \widetilde{\mathbf{T}}_{\lambda^{\prime}, \mathcal{K}_{1}}^{\prime}
\end{gathered}
$$

Let $\widetilde{\mathbf{T}}_{\theta^{\prime}}^{\prime}$, resp. $\widetilde{\mathbf{T}}_{\lambda^{\prime}}^{\prime}$, be the image of $\widetilde{\mathbf{T}}_{3}^{u}$ by the second projection in $\left(2^{\prime}\right)$, resp. in $\left(3^{\prime}\right)$. Besides the ideal $\mathfrak{c}_{\mu}$ already defined, one can define two other congruence ideals :

and

$$
\mathfrak{c}_{\theta^{\prime}}=\widetilde{\mathbf{T}}_{3}^{u} \cap\left(\widetilde{\mathbf{T}}_{2}^{s} \times\left\{0_{\widetilde{\mathbf{T}}_{\theta^{\prime}}^{\prime}}\right\}\right)
$$

$$
\mathfrak{c}_{\lambda^{\prime}}=\widetilde{\mathbf{T}}_{3}^{u} \cap\left(\widetilde{A}_{1} \times\left\{0_{\widetilde{\mathbf{T}}_{\lambda^{\prime}}^{\prime}}\right\}\right) .
$$

The formalism of Sections 8.3-8.5 yields the following 
Corollary 3.6. Assume (*).

1) Assume either $\alpha^{12} \not \equiv 1\left(\bmod \mathfrak{m}_{A_{1}}\right)$ or $3(a+1)<p-1$, then the ideals $\mathfrak{c}_{\lambda^{\prime}}, \mathfrak{c}_{\lambda} \lambda\left(\mathfrak{c}_{\theta^{\prime}}\right)$ and $\mathfrak{c}_{\theta^{\prime}}$ are principal and we have the relation

$$
\mathfrak{c}_{\lambda^{\prime}}=\mathfrak{c}_{\lambda} \lambda\left(\mathfrak{c}_{\theta^{\prime}}\right) .
$$

2) If one assumes $3(a+1)<p-1$, one has moreover that $\mathfrak{c}_{\lambda^{\prime}}$ is generated by $\operatorname{Char}\left(\operatorname{Sel}\left(\operatorname{Ad}_{\mathfrak{s p}} \rho_{\mu}\right)\right)$,

Proof. Same proof as in Corollary 3.2.

Note that the associated primes of $\lambda\left(\mathfrak{c}_{\theta^{\prime}}\right)$ in $\widetilde{A}_{1}$ are congruence primes between $\operatorname{Symm}^{3}(\mu)$ and unitary families which don't come from Siegel families.

Moreover, according to the Greenberg-Iwasawa main conjecture, the ideal $\lambda\left(\mathfrak{c}_{\theta^{\prime}}\right)$ should be generated by the (still conjectural) $p$-adic $L$ function $L_{p}\left(\mathcal{A}_{\mu}^{2}\right)$. On the other hand, for $p>3$, we have a decomposition of $\mathbb{Z}_{p}$-representations of $\mathrm{GL}_{2}: \mathrm{Ad}_{\mathfrak{s} \mathfrak{I}_{4}}=\mathrm{Ad}_{\mathfrak{s p}} \oplus \mathcal{A}^{2}$. This implies a decomposition of minimal $p$-ordinary Selmer groups

$$
\operatorname{Sel}\left(\operatorname{Ad}_{\mathfrak{s} l_{4}} \rho_{\mu}\right)=\operatorname{Sel}\left(\operatorname{Ad}_{\mathfrak{s p}} \rho_{\mu}\right) \oplus \operatorname{Sel}\left(\mathcal{A}_{\mu}^{2}\right)
$$

Since we know that $\mathfrak{c}_{\lambda}=\operatorname{Char}\left(\operatorname{Sel}\left(\operatorname{Ad}_{\mathfrak{s p}_{4}} \rho_{\mu}\right)^{*}\right)$, we conclude by division :

Corollary 3.7. The ideal $\lambda\left(\mathfrak{c}_{\theta^{\prime}}\right)$ is principal generated by $\operatorname{Char}\left(\operatorname{Sel}\left(\mathcal{A}_{\mu}^{2}\right)\right)^{*}$.

Proof. Same proof as in Corollary 3.2.

\section{The CASE $j=4$}

To treat this case, we fix an auxiliary imaginary quadratic field as above and we choose unitary groups $U(4)$ and $U(5)$ which are compact at infinity and with the same local conditions at finite places.

Besides the Symm ${ }^{3}$ base change, we also consider the $\mathrm{Symm}^{4}$ base change from $\mathrm{GL}_{2}$ (established by $\mathrm{H}$. Kim[Kim03] to $\mathrm{GL}_{5}$, and by Clozel to $\left.U(5)\right)$. We note the commutative diagram of group schemes over $\mathbb{Z}_{p}$ :

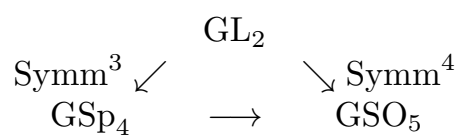

where the bottom arrow is the standard $(2: 1)$-covering coming from the exceptional isomorphism $\mathrm{GSp}(4) \cong \mathrm{GSpin}_{5}$. Recall that by definition $\mathrm{GSO}_{5}=\mathbb{G}_{m} \times \mathrm{SO}_{5}$. Therefore, the adjoint action of Symm ${ }^{4} \mathrm{GL}_{2}$ on $\mathfrak{s o}_{5}$ coincides with the adjoint action of $\mathrm{Symm}^{3} \mathrm{GL}_{2}$ on $\mathfrak{s p}_{4}$. We therefore have the following $\mathbb{Z}_{p}$-decompositions for the action of $\operatorname{Symm}^{4} \mathrm{GL}_{2}$ :

$$
\mathfrak{s o}_{5}=\mathcal{A}^{1} \oplus \mathcal{A}^{3}
$$

and

$$
\mathfrak{s l}_{5}=\mathcal{A}^{1} \oplus \mathcal{A}^{2} \oplus \mathcal{A}^{3} \oplus \mathcal{A}^{4} .
$$

Let $R_{4}$ be the minimal $p$-ordinary universal ring of deformations $\rho: G_{\mathbb{Q}} \rightarrow \mathcal{G}_{5}(B)$ of $\operatorname{Symm}^{4} \bar{\rho}_{\mu}$. Since 5 is odd, one can choose an imaginary quadratic field in which $p$ and $q_{1}$ split, a skewfield $D$ split outside $\mathfrak{q}_{1}$ and $q G_{1}^{c}$ and a second kind involution $*$ on $D$ such that $G=U(D, *)$ is definite at $\infty$ and quasisplit at all inert places. Again, by Geraghty's thesis, the Hida Hecke algebra $h_{4}$ associated to $U(5)$ is finite torsion free $\Lambda_{4}$. Its localization $\mathbf{T}_{4}^{u}$ at the maximal ideal associated to $\operatorname{Symm}^{4} \bar{\rho}_{\mu}$ satisfies

Theorem 4.1. Assuming $\alpha^{12} \not \equiv 1\left(\bmod \mathfrak{m}_{A_{1}}\right)$ or $4(a+1)<p-1$, we have $R_{4}=\mathbf{T}_{4}^{u}$; this ring is local complete intersection over $\Lambda_{4}$. In particular it is finite flat over $\Lambda_{4}$.

Note that the universal morphism $R_{4} \rightarrow R_{2}$ induced by the diagram (4) gives rise by identification to a non obvious base change morphism $\theta^{\prime \prime}: \mathbf{T}_{4}^{u} \rightarrow \mathbf{T}_{2}^{s}$ above the morphism $\Lambda_{4} \rightarrow \Lambda_{2}$ (also induced by the diagram (4)). Let $\widetilde{\mathbf{T}}_{4}^{u}=\mathbf{T}_{4}^{u} \otimes_{\Lambda_{4}} \widetilde{A}_{1}$. We consider the morphisms

$$
\widetilde{\mathbf{T}}_{4}^{u} \stackrel{\theta^{\prime \prime}}{\rightarrow} \widetilde{\mathbf{T}}_{2}^{s} \stackrel{\lambda}{\rightarrow} \widetilde{A}_{1}
$$


Let $\lambda^{\prime \prime}=\lambda \circ \theta^{\prime \prime}$. We can define the congruence ideal $\mathfrak{c}_{\lambda^{\prime \prime}}$ as before by

$$
\mathfrak{c}_{\lambda^{\prime \prime}}=\widetilde{\mathbf{T}}_{4}^{u} \cap\left(\widetilde{A}_{1} \times\left\{0_{\widetilde{\mathbf{T}}_{\lambda^{\prime \prime}}^{\prime}}\right\}\right) .
$$

Corollary 4.2. Assume $4(a+1)<p-1$. The ideal $\mathfrak{c}_{\lambda^{\prime \prime}}$ is principal generated by a characteristic power series of $\operatorname{Sel}\left(\operatorname{Ad}_{\mathfrak{s i} 5} \operatorname{Symm}^{4} \rho_{\mu}\right)^{*}$.

Proof. Same proof as in Corollary 3.2.

We define a new congruence ideal $\mathfrak{c}_{\theta^{\prime \prime}}$ by

$$
\mathfrak{c}_{\theta^{\prime \prime}}=\widetilde{\mathbf{T}}_{4}^{u} \cap\left(\widetilde{\mathbf{T}}_{2}^{s} \times\left\{0_{\widetilde{\mathbf{T}}_{\theta^{\prime \prime}}^{\prime}}\right\}\right) .
$$

Then,

Corollary 4.3. We have

$$
\mathfrak{c}_{\lambda^{\prime \prime}}=\mathfrak{c}_{\lambda} \lambda\left(\mathfrak{c}_{\theta^{\prime \prime}}\right)
$$

Moreover, the congruence ideal $\lambda\left(\mathfrak{c}_{\theta^{\prime}}\right)$ divides $\lambda\left(\mathfrak{c}_{\theta^{\prime \prime}}\right)$ and

$$
\frac{\lambda\left(\mathfrak{c}_{\theta^{\prime \prime}}\right)}{\lambda\left(\mathfrak{c}_{\theta^{\prime}}\right)}=\operatorname{Char}\left(\operatorname{Sel}\left(\mathcal{A}_{\mu}^{4}\right)^{*}\right) .
$$

Proof. We have a decomposition of Selmer groups

$$
\operatorname{Sel}\left(\operatorname{Ad}_{\mathfrak{s i}_{5}} \operatorname{Symm}^{4} \rho_{\mu}\right)=\operatorname{Sel}\left(\operatorname{Ad}_{\mathfrak{s} 0_{5}} \operatorname{Symm}^{4} \rho_{\mu}\right) \oplus \operatorname{Sel}\left(\mathcal{A}_{\mu}^{2}\right) \oplus \operatorname{Sel}\left(\mathcal{A}_{\mu}^{4}\right)
$$

Of course this suggests that the prime factors in $\widetilde{A}_{1}$ of the (not yet constructed) $p$-adic $L$ function $L_{p}\left(\mathcal{A}_{\mu}^{4}\right)$ are congruence primes between $\operatorname{Symm}^{4} \mu$ and forms on $U(5)$ which don't come from $\operatorname{GSp}(4)$ (by the base change given by $\mathrm{GSpin}_{5} \rightarrow \mathrm{GSO}_{5}$ ).

\section{Digression: a Kummer type Criterion for the non triviality of Certain Selmer} GROUPS

We keep the notations of the introduction and we assume $(*)$. Let $\mathfrak{p}$ be a prime of $\widetilde{A}_{1}$. For $j=3,2,4$, consider the condition

$$
\left(S_{j}\right) \quad \operatorname{Fitt}_{0}\left(\operatorname{Sel}\left(\mathcal{A}_{\mu}^{j}\right)^{*}\right) \subset \mathfrak{p}
$$

and the condition

$\left(C_{3}\right)$ there exists a Hida family $G$ of Iwahori level $N$ on $\mathrm{GSp}_{4}$ which is not the $\mathrm{Symm}^{3}$ of a Hida family on $\mathrm{GL}_{2}$ and such that $\mathrm{Symm}^{3} \mu \equiv G(\bmod \mathfrak{p})$.

We prove

Theorem 5.1. Assume $(*)$, then $\left(C_{3}\right)$ implies $\left(S_{3}\right)$ or $\left(S_{2}\right)$ or $\left(S_{4}\right)$.

Proof. Let $\nu_{G}: \mathbf{T}_{2} \rightarrow A_{2}$ be the Hida family associated to $G$ and $\rho_{G}: \Gamma_{\mathbb{Q}} \rightarrow \operatorname{GSp}_{4}\left(A_{2}\right)$ be the Galois representation associated to this Hida family. It is well defined because $\bar{\rho}_{G}=\operatorname{Symm}^{3} \bar{\rho}_{\mu}$ is absolutely irreducible by $(*)$. We define $\widetilde{\mathbf{T}}_{1}=\mathbf{T}_{1} \otimes_{\Lambda_{1}} \widetilde{A}_{1}$ and $\widetilde{\mathbf{T}}_{2}=\mathbf{T}_{2} \otimes_{\Lambda_{2}} \widetilde{A}_{1}$ and we consider

$$
\widetilde{\mathbf{T}}_{2} \stackrel{\widetilde{\sigma}_{3}}{\rightarrow} \widetilde{\mathbf{T}}_{1} \stackrel{\widetilde{\mu}}{\rightarrow} \widetilde{A}_{1}
$$

We can decompose $A_{2} \otimes_{\Lambda_{2}} \widetilde{A}_{1}$ as a product of domains $B_{i}$ which are finite extensions of $\widetilde{A}_{1}$. Let $C$ be the normalisation of $B_{1}$. By assumption, $\rho_{G, C}=\rho_{G} \otimes_{A_{2}} C$ is not a $\mathrm{Symm}^{3}$ but there exists a prime ideal $\mathfrak{p}_{C}$ of $C$ above $\mathfrak{p}$ such that, denoting by $\phi$, resp. $\iota$, the homomorphism $\phi: C \rightarrow C / \mathfrak{p}_{C}$, resp. $\iota: \widetilde{A}_{1} / \mathfrak{p} \hookrightarrow C / \mathfrak{p}_{C}$, we have $\phi_{*} \rho_{G, C}=\iota_{*} \operatorname{Symm}^{3} \rho_{\mu}(\bmod \mathfrak{p})$ up to conjugation in $C / \mathfrak{p}_{C}$. Note that $\mathfrak{p}_{C}=\operatorname{Ker} \phi$ is a height one prime of the normal ring $C$. Since $\rho_{G, C}$ is not the Symm ${ }^{3}$ of a Hida family on $\mathrm{GL}_{2}$, it follows by a theorem of Conti [Con16b] that $\mathrm{Im} \rho_{G, C}$ contains a congruence subgroup of $\operatorname{GSp}_{4}\left(\Lambda_{1}\right)$ up to conjugation by an element of $\operatorname{GSp}_{4}(C)$. In particular, the adjoint action $\operatorname{Ad}^{0} \rho_{G, C}$ on $\mathfrak{s p}_{4}(C)$ is irreducible while it becomes reducible when one applies $\phi$. More precisely:

$$
\phi_{*}\left(\operatorname{Ad}^{0} \rho_{G, C}\right) \cong \mathcal{A}_{\mu}^{1}\left(C / \mathfrak{p}_{C}\right) \oplus \mathcal{A}_{\mu}^{3}\left(C / \mathfrak{p}_{C}\right)
$$


Note that $C_{\mathfrak{p}_{C}}$ is a dvr. Let $K=\operatorname{Frac}\left(C / \mathfrak{p}_{C}\right)$. By [Ri76, Proposition 4.2], there exists a $C_{\mathfrak{p}_{C}}$-lattice $L$ in $\mathfrak{s p}_{4}(\operatorname{Frac}(C))$ with Galois action such that on the quotient $L / \mathfrak{p}_{C} L$, the action of Galois is a non trivial extension of $\mathcal{A}_{\mu}^{1}(K)$ by $\mathcal{A}_{\mu}^{3}(K)$. But $\operatorname{Hom}\left(\mathcal{A}_{\mu}^{1}(K), \mathcal{A}_{\mu}^{3}(K)\right)=\mathcal{A}_{\mu}^{3}(K) \oplus \mathcal{A}_{\mu}^{2}(K) \oplus \mathcal{A}_{\mu}^{4}(K)$ as $\Gamma_{\mathbb{Q}}$-modules. Since we are dealing with $N$-minimal $p$-ordinary Selmer groups, the non triviality of one of the three Selmer groups over $K$ follows.

\section{THE CASE $j=n$}

It follows from [PT02] that for any $n$ with $1 \leq n<p-1$, we have a decomposition $\mathfrak{s l}_{n+1}=$ $\bigoplus_{j=1}^{n} \mathcal{A}^{j}$ over $\mathbb{Z}_{p}$ which is $\mathrm{GL}_{2}$-equivariant for the action on the left hand side by $\mathrm{AdSymm}^{n}$. It follows that for $n \in[1, p-1[$, we have

$$
\mathfrak{s l}_{n+1}=\mathfrak{s l}_{n} \oplus \mathcal{A}^{n}
$$

where $\mathrm{GL}_{2}$ acts by $\mathrm{Ad} \mathrm{Symm}^{n}$ on the left hand side and by $\mathrm{Ad} \mathrm{Symm}^{n-1}$ on the first factor or the right-hand side.

From now on, we take $j=n-1$ or $j=n$ Let $h_{j}^{u}$ be the Hida Hecke algebra associated to the unitary group $U(j+1)$ chosen to be compact at infinity and with local conditions af finite primes as before. This algebra is finite torsion free over the Iwasawa algebra $\Lambda_{j}$ in $j$ variables. Let us assume the automorphic base change is established for $\operatorname{Symm}^{j}$ for $j=n-1$ and $j=n$. This gives rise to algebra homomorphisms $\theta_{j}: h_{j}^{u} \rightarrow h_{1}$ above the homomorphism $\Lambda_{j} \rightarrow \Lambda_{1}$ induced by $m \geq 0 \mapsto\left(x_{1}, \cdots, x_{j}\right)$ with $x_{1}=m, x_{2}=2 m, \ldots, x_{j}=j m$. Let $\mu: h_{1} \rightarrow A_{1}$ be a Hida family and let $\lambda_{j}=\mu \circ \theta_{j}$, for $j=n-1, n$. We assume that the image of the residual representation $\bar{\rho}_{\mu}$ contains $\mathrm{SL}_{2}\left(\mathbb{F}_{p}\right)$. Let $\mathbf{T}_{j}^{u}$ be the localization of $h_{j}^{u}$ at the maximal ideal associated to $\operatorname{Symm}^{j} \bar{\rho}_{\mu}$. Let $R_{j}$ be the universal deformation ring for minimal $p$-ordinary lifts $\rho: G_{\mathbb{Q}} \rightarrow \mathcal{G}_{j+1}(B)$ of $\operatorname{Symm}^{j} \bar{\rho}_{\mu}$. We have proven in Section 2

Theorem 6.1. Assuming $(*), n(a+1)<p-1$, and that the transfer Symm $^{j}$ is established for $j=n-1, n$, then for $j=n-1, n$, we have $R_{j}=\mathbf{T}_{j}^{u}$ and these rings are local complete intersection over $\Lambda_{j}$.

From this it follows that for $j=n-1, n$, the congruence modules $\mathfrak{c}_{\lambda_{j}}$ and $\mathfrak{c}_{\theta_{j}}$ are principal and related by the relation

$$
\mathfrak{c}_{\lambda_{j}}=\lambda_{j}\left(\mathfrak{c}_{\theta_{j}}\right) \mathfrak{c}_{\mu}
$$

Moreover it also follows from the theorem that for $j=n-1, n$, the ideal $\mathfrak{c}_{\lambda_{j}}$ is generated by Char $\left.\left(\left(\operatorname{Sel}\left(\operatorname{Ad~} \operatorname{Symm}^{j} \rho_{\mu}\right)\right)^{*}\right)\right)$. Since we have

$$
\left.\left.\operatorname{Sel}\left(\operatorname{Ad} \operatorname{Symm}^{j} \rho_{\mu}\right)\right)=\operatorname{Sel}\left(\operatorname{Ad} \operatorname{Symm}{ }^{j-1} \rho_{\mu}\right)\right) \oplus \operatorname{Sel}\left(\mathcal{A}_{\mu}^{j}\right)
$$

we deduce by passing to the characteristic power series of the Pontryagin duals that

$$
\mathfrak{c}_{\lambda_{n}}=\mathfrak{c}_{\lambda_{n-1}}\left(\operatorname{Char}\left(\operatorname{Sel} \mathcal{A}_{\mu}^{n}\right)\right)
$$

and dividing by the invertible ideal $\mathfrak{c}_{\mu}$, we conclude that $\lambda_{n-1}\left(\mathfrak{c}_{\theta_{n-1}}\right)$ divides $\lambda_{n}\left(\mathfrak{c}_{\theta_{n}}\right)$ and that the quotient is the principal ideal $\left(\operatorname{Char}\left(\operatorname{Sel} \mathcal{A}_{\mu}^{n}\right)\right)$.

This theorem applies to $n=4,5,6,7,8$ since the transfers $\mathrm{Symm}^{j}, j=3,4,5,6,7,8$ of a classical form of weight $\geq 2$ have been established in [CT15]. For $n=4$, we obtain a different proof of the Theorem 4.3 given in 4 relating congruences on $U(4)$ and $U(5)$. In that case, the congruence ideals refer to transfers from $\operatorname{GSp}(4)$ to $U(4)$ and $U(5)$, while here they refer to the congruences between Symm $^{3}$, resp. Symm ${ }^{4}$ transfers and families on $U(4)$ resp. $U(5)$. For $n \geq 5$, there is no alternative proof because there is no known transfer from $\mathrm{GSp}_{4}$ to $U(n+1)$ compatible to $\mathrm{Symm}^{3}$ and $\mathrm{Symm}^{n}$.

The meaning of this is that any congruence prime between $\operatorname{Symm}^{n-1} \mu$ and a family of $U(n)-$ forms which are not $\mathrm{Symm}^{n-1}$ fromGL 2 is also a congruence prime between $\mathrm{Symm}^{n} \mu$ and a family of $U(n+1)$-forms which are not $\mathrm{Symm}^{n}$ from $\mathrm{GL}_{2}$. However, it doesn't seem that one can define a cuspidal base change from $U(n)$ to $U(n+1)$ which would explain this phenomenon by Tate-Hida formalism. It appears for the moment only as a consequence of our congruence ideal main conjecture theorem. 


\section{The CASE of the Standard RePresentation of GSp(4)}

This is a digression which does not involve symmetric powers of $\mathrm{GL}_{2}$. Let instead consider a Hida family of Siegel cusp forms that is, a $\Lambda_{2}$-algebra homomorphism $\sigma: h_{2}^{s} \rightarrow A_{2}$ onto a domain $A_{2}$ which is finite and torsion free over $\Lambda_{2}$. As we noted above, using the base change from GSp(4) to GL(4) established in [Mok14] and Clozel's descent to $U(4)$, we constructed a morphism $\theta^{\prime}: h_{3}^{u} \rightarrow h_{2}^{s}$. We assume that the residual Galois representation $\bar{\rho}_{\sigma}$ is absolutely irreducible. Moreover by ordinarity the restriction of $\bar{\rho}_{\sigma}$ to an inertia group $I_{p}$ at $p$ is conjugate to

$$
\left(\begin{array}{cccc}
1 & * & * & * \\
& \omega^{-\left(a_{2}+1\right)} & * & * \\
& & \omega^{-\left(a_{1}+2\right)} & * \\
& & & \omega^{-\left(a_{1}+a_{2}+3\right)}
\end{array}\right)
$$

for a pair of integers $a_{1} \geq a_{2} \geq 0$. We assume $a_{1}+a_{2}+3<p-1$. There can be only one pair $\left(a_{1}, a_{2}\right)$ of integers such that $a_{1} \geq a_{2} \geq 0$ and $a_{1}+a_{2}+3<p-1$. It is fixed from now on.

We localize the morphism $\theta^{\prime}$ at maximal ideals associated to $\bar{\rho}_{\sigma}$. We write $\theta^{\prime}: \mathbf{T}_{3}^{u} \rightarrow \mathbf{T}_{2}^{s}$ for its localization. If the residual image is big (in the sense of [Pi12b] Section 5.8), and that the four Hecke eigenvalues at $p$ are distinct modulo $\mathfrak{m}_{A_{1}}$. Then, let $R_{i} i=2,3$ be the minimal $p$-ordinary universal deformation rings of $\bar{\rho}_{\sigma}$ (for deformations into $\operatorname{GSp}_{4}(B)$ resp. $\mathcal{G}_{4}(B)$ ), we can prove

Theorem 7.1. Assume $a_{1}+a_{2}+3<p-1$; then we have $R_{2}=\mathbf{T}_{2}^{s}$ and $R_{3}=\mathbf{T}_{3}^{u}$, and the rings $\mathbf{T}_{2}^{s}$ resp. $\mathbf{T}_{3}^{u}$ is local complete intersection over $\Lambda_{2}$ resp. $\Lambda_{3}$.

If we set $\lambda=\sigma \circ \theta^{\prime}$, we can define three congruence ideals $\mathfrak{c}_{\lambda}, \mathfrak{c}_{\sigma} \mathfrak{c}_{\theta}^{\prime}$. Because of the assumption $a_{1}+a_{2}+3<p-1$, we see as in the proof of Proposition 3.3 that the differential module $\Omega_{\mathbf{T}_{3}^{u} / \Lambda_{2}} \otimes_{\mathbf{T}_{3}^{u}} \widetilde{A}_{2}$ is isomorphic to $\left.\operatorname{Sel}\left(\operatorname{Ad}_{\mathfrak{s} l_{4}} \rho_{\sigma}\right)\right)^{*}$ and that similarly $\Omega_{\mathbf{T}_{2}^{s} / \Lambda_{2}} \otimes_{\mathbf{T}_{2}^{s}} \widetilde{A}_{2}$ is isomorphic to $\left.\operatorname{Sel}\left(\operatorname{Ad}_{\mathfrak{s p}} \rho_{\sigma}\right)\right)^{*}$. Hence by Theorem 8.7, we conclude that $\widetilde{\mathfrak{c}_{\lambda}}=\operatorname{Char}\left(\left(\operatorname{Sel}\left(\operatorname{Ad}_{\mathfrak{s} r_{4}} \rho_{\sigma}\right)\right)^{*}\right)$ and $\widetilde{\mathfrak{c}_{\sigma}}=\operatorname{Char}\left(\left(\operatorname{Sel}\left(\operatorname{Ad}_{\mathfrak{s p}_{4}} \rho_{\sigma}\right)\right)^{*}\right)$. We also have the transfer formula of Proposition 8.14 :

$$
\widetilde{\mathfrak{c}_{\lambda}}=\widetilde{\mathfrak{c}_{\sigma} \sigma\left(\mathfrak{c}_{\theta^{\prime}}\right)} \text {. }
$$

On the other hand, we have

$$
\operatorname{Ad}_{\mathfrak{s l}_{4}}\left(\rho_{\sigma}\right)=\operatorname{Ad}_{\mathfrak{s p}_{4}}\left(\rho_{\sigma}\right) \oplus \operatorname{St}_{\sigma}
$$

where $\mathrm{St}_{\sigma}$ is the composition of $\sigma$ with $\mathrm{St}: \mathrm{GSp}_{4} \rightarrow \mathrm{GSO}_{5}$. From this and Proposition 8.14 (for $\nu=2$ ) and Theorem 8.15, we conclude

Theorem 7.2. The reflexive envelope $\widetilde{\sigma\left(\mathfrak{c}_{\theta^{\prime}}\right)}$ of the ideal $\sigma\left(\mathfrak{c}_{\theta^{\prime}}\right)$ of $\widetilde{A}_{2}$ is principal and is generated by $\operatorname{Char}\left(\left(\operatorname{Sel}\left(\mathrm{St}_{\sigma}\right)\right)^{*}\right)$.

Remark 7.3. The p-adic $L$ function $L_{p}\left(\mathrm{St}_{\sigma}\right)$ has been constructed by Zheng Liu in her 2016 Columbia thesis, and the main conjecture implies that $\sigma\left(\mathfrak{c}_{\theta^{\prime}}\right)$ is also generated by $L_{p}\left(\mathrm{St}_{\sigma}\right)$. It is therefore natural to ask whether the height one prime factors of $L_{p}\left(\mathrm{St}_{\sigma}\right)$ in $\widehat{A}_{2}$ are congruence primes between $\sigma$ and families on $U(4)$ which don't come from $\operatorname{GSp}(4)$.

\section{Congruence ideal Formalism}

We recall a formalism developed by Hida based on Tate's appendix to [MR70], alongside we introduce the notion of congruence modules and differential modules for general rings and basic facts about it. We apply the theory to Hecke algebras and deformation rings to show that these two torsion modules have the same size (that is, the equal characteristic ideals and Fitting ideals).

8.1. Differentials. We recall here the definition of 1-differentials and some of their properties for our later use. Let $R$ be a $A$-algebra, and suppose that $R$ and $A$ are objects in $C N L_{W}$, where $W$ is a finite flat extenion of $\mathbb{Z}_{p}$ The module of 1 -differentials $\Omega_{R / A}$ for a $A$-algebra $R\left(R, A \in C N L_{W}\right)$ indicates the module of continuous 1 -differentials with respect to the profinite topology. 
For a module $M$ with continuous $R$-action (in short, a continuous $R$-module), let us define the module of A-derivations by

$$
\operatorname{Der}_{A}(R, M)=\left\{\begin{array}{c}
\delta: R \rightarrow M \in \operatorname{Hom}_{A}(R, M) \mid \begin{array}{c}
\delta: \text { continuous } \\
\delta(a b)=a \delta(b)+b \delta(a) \\
\text { for all } a, b \in R
\end{array}
\end{array}\right\} .
$$

Here the $A$-linearity of a derivation $\delta$ is equivalent to $\delta(A)=0$, because

$$
\delta(1)=\delta(1 \cdot 1)=2 \delta(1) \Rightarrow \delta(1)=0 .
$$

Then $\Omega_{R / A}$ represents the covariant functor $M \mapsto \operatorname{Der}_{A}(R, M)$ from the category of continuous $R$-modules into $M O D$.

The construction of $\Omega_{R / A}$ is easy. Let $R \widehat{\otimes}_{A} R$ be the completion of $R \otimes_{A} R$ with respect to the $\left(\mathfrak{m}_{R} \otimes_{A} R+R \otimes_{A} \mathfrak{m}_{R}\right)$-adic topology. The multiplication $a \otimes b \mapsto a b$ induces a $A$-algebra homomorphism $m: R \widehat{\otimes}_{A} R \rightarrow R$ taking $a \otimes b$ to $a b$. We put $I=\operatorname{Ker}(m)$, which is an ideal of $R \widehat{\otimes}_{A} R$. Then we define $\Omega_{R / A}=I / I^{2}$. We endow it with a structure of $R$-module by action of $R \widehat{\otimes} 1$. It is a complete module for the $\mathfrak{m}_{R}$-topology. One checks that the map $d: R \rightarrow \Omega_{R / A}$ given by $d(a)=a \otimes 1-1 \otimes a \bmod I^{2}$ is a continuous $A$-derivation. Thus we have a morphism of functors: $\operatorname{Hom}_{R}\left(\Omega_{R / A}, ?\right) \rightarrow \operatorname{Der}_{A}(R, ?)$ given by $\phi \mapsto \phi \circ d$. Since $\Omega_{R / A}$ is generated by $d(R)$ as $R$-modules (left to the reader as an exercise), the above map is injective. To show that $\Omega_{R / A}$ represents the functor, we need to show the surjectivity of the above map, which is well known (see [CRT, ]).

Proposition 8.1. The above morphism of two functors $M \mapsto \operatorname{Hom}_{R}\left(\Omega_{R / A}, M\right)$ and $M \mapsto \operatorname{Der}_{A}(R, M)$ is an isomorphism, where $M$ runs over the category of complete $R$-modules. In other words, for each $A$-derivation $\delta: R \rightarrow M$, there exists a unique $R$-linear homomorphism $\phi: \Omega_{R / A} \rightarrow M$ such that $\delta=\phi \circ d$.

We have the following fundamental exact sequences:

Corollary 8.2. Let the notation be as in the proposition.

(i) Suppose that $A$ is a $C$-algebra for an object $C \in C L_{W}$. Then we have the following natural exact sequence:

$$
\Omega_{A / C} \widehat{\otimes}_{A} R \longrightarrow \Omega_{R / C} \longrightarrow \Omega_{R / A} \rightarrow 0 .
$$

(ii) Let $\pi: R \rightarrow C$ be a surjective morphism in $C L_{W}$, and write $J=\operatorname{Ker}(\pi)$. Then we have the following natural exact sequence:

$$
J / J^{2} \stackrel{\beta^{*}}{\rightarrow} \Omega_{R / A} \widehat{\otimes}_{R} C \longrightarrow \Omega_{C / A} \rightarrow 0 .
$$

Moreover if $A=C$, then $J / J^{2} \cong \Omega_{R / A} \widehat{\otimes}_{R} C$.

For any continuous $R$-module $M$, we write $R[M]$ for the $R$-algebra with square zero ideal $M$. Thus $R[M]=R \oplus M$ with the multiplication given by

$$
(r \oplus x)\left(r^{\prime} \oplus x^{\prime}\right)=r r^{\prime} \oplus\left(r x^{\prime}+r^{\prime} x\right) .
$$

It is easy to see that $R[M] \in C N L_{W}$, if $M$ is of finite type, and $R[M] \in C L_{W}$ if $M$ is a $p$-profinite $R$-module. By definition,

$$
\operatorname{Der}_{A}(R, M) \cong\left\{\phi \in \operatorname{Hom}_{A-a l g}(R, R[M]) \mid \phi \quad \bmod M=\mathrm{id}\right\},
$$

where the map is given by $\delta \mapsto\left(a \mapsto(a \oplus \delta(a))\right.$. Note that $i: R \rightarrow R \widehat{\otimes}_{A} R$ given by $i(a)=a \otimes 1$ is a section of $m: R \widehat{\otimes}_{A} R \rightarrow R$. We see easily that $R \widehat{\otimes}_{A} R / I^{2} \cong R\left[\Omega_{R / A}\right]$ by $x \mapsto m(x) \oplus(x-i(m(x)))$. Note that $d(a)=1 \otimes a-i(a)$ for $a \in R$. 
8.2. Congruence and differential modules. Let $R$ be an algebra over a normal noetherian domain $A$. We assume that $R$ is an $A$-flat module of finite type. Let $\phi: R \rightarrow A$ be an $A$-algebra homomorphism. We define

$$
C_{1}(\phi ; A)=\Omega_{R / A} \otimes_{R, \phi} \operatorname{Im}(\phi)
$$

which we call the differential module of $\phi$. We have seen (for instance Corollary 3.2, (2)) that if $R$ is a deformation ring, this module is the dual of the associated adjoint Selmer group. If $\phi$ is surjective, we just have

$$
C_{1}(\phi ; A)=\Omega_{R / A} \otimes_{R, \phi} A .
$$

We suppose that $R$ is reduced (having zero nilradical of $R$ ). Then the total quotient $\operatorname{ring} \operatorname{Frac}(R)$ can be decomposed uniquely into $\operatorname{Frac}(R)=\operatorname{Frac}(\operatorname{Im}(\phi)) \oplus X$ as an algebra direct product. Write $1_{\phi}$ for the idempotent of $\operatorname{Frac}(\operatorname{Im}(\phi))$ in $\operatorname{Frac}(R)$. Let $\mathfrak{a}=\operatorname{Ker}(R \rightarrow X)=\left(1_{\phi} R \cap R\right), S=\operatorname{Im}(R \rightarrow X)$ and $\mathfrak{b}=\operatorname{Ker}(\phi)$. Here the intersection $1_{\phi} R \cap R$ is taken in $\operatorname{Frac}(R)=\operatorname{Frac}(\operatorname{Im}(\phi)) \oplus X$. Then we put

$$
C_{0}(\phi ; A)=(R / \mathfrak{a}) \otimes_{R, \phi} \operatorname{Im}(\phi) \cong \operatorname{Im}(\phi) /(\phi(\mathfrak{a})) \cong 1_{\phi} R / \mathfrak{a} \cong S / \mathfrak{b} \cong R /(\mathfrak{a} \oplus \mathfrak{b}),
$$

which is called the congruence module of $\phi$ but is actually a ring (cf. [H88b] Section 6). We can split the isomorphism $1_{\phi} R / \mathfrak{a} \cong S / \mathfrak{b}$ as follows: First note that $\mathfrak{a}=\left(R \cap\left(1_{\phi} R \oplus 0\right)\right)$ in $\operatorname{Frac}(\operatorname{Im}(\phi)) \oplus X$. Then $\mathfrak{b}=(0 \oplus X) \cap R$, and we have

$$
1_{\phi} R / \mathfrak{a} \cong R /(\mathfrak{a} \oplus \mathfrak{b}) \cong S / \mathfrak{b}
$$

where the maps $R /(\mathfrak{a} \oplus \mathfrak{b}) \rightarrow 1_{\phi} R / \mathfrak{a}$ and $R /(\mathfrak{a} \oplus \mathfrak{b}) \rightarrow S / \mathfrak{b}$ are induced by two projections from $R$ to $1_{\phi} R$ and $S$.

Write $K=\operatorname{Frac}(A)$. Fix an algebraic closure $\bar{K}$ of $K$. Since the spectrum $\operatorname{Spec}\left(C_{0}(\phi ; A)\right)$ of the congruence ring $C_{0}(\phi ; A)$ is the scheme theoretic intersection of $\operatorname{Spec}(\operatorname{Im}(\phi))$ and $\operatorname{Spec}(R / \mathfrak{a})$ in $\operatorname{Spec}(R)$ :

$$
\operatorname{Spec}\left(C_{0}(\lambda ; A)\right)=\operatorname{Spec}(\operatorname{Im}(\phi)) \cap \operatorname{Spec}(R / \mathfrak{a}):=\operatorname{Spec}(\operatorname{Im}(\phi)) \times \operatorname{Spec}(R) \operatorname{Spec}(R / \mathfrak{a}),
$$

we conclude that

Proposition 8.3. Let the notation be as above. Then a prime $\mathfrak{p}$ is in the support of $C_{0}(\phi ; A)$ if and only if there exists an A-algebra homomorphism $\phi^{\prime}: R \rightarrow \bar{K}$ factoring through $R / \mathfrak{a}$ such that $\phi(a) \equiv \phi^{\prime}(a) \bmod \mathfrak{p}$ for all $a \in R$.

In other words, $\phi \bmod \mathfrak{p}$ factors through $R / \mathfrak{a}$ and can be lifted to $\phi^{\prime}$. Therefore, if $A$ is the integer ring of a sufficiently large number field in $\overline{\mathbb{Q}}, \bigcup_{\phi} \operatorname{Supp}\left(C_{0}(\phi ; A)\right)$ is made of primes dividing the absolute different $\mathfrak{d}(R / \mathbb{Z})$ of $R$ over $\mathbb{Z}$, and each prime appearing in the absolute discriminant of $R / \mathbb{Z}$ divides the order of the congruence module for some $\phi$.

By Corollary 8.2 applied to the exact sequence: $0 \rightarrow \mathfrak{b} \rightarrow R \stackrel{\phi}{\rightarrow} A \rightarrow 0$, we know that

$$
C_{1}(\phi ; A) \cong \mathfrak{b} / \mathfrak{b}^{2} .
$$

Since $C_{0}(\phi ; A) \cong S / \mathfrak{b}$, we may further define higher congruence modules by $C_{n}(\phi ; A)=\mathfrak{b}^{n} / \mathfrak{b}^{n+1}$.

8.3. Transfer property of congruence modules. Let $B$ be a normal profinite local domain of characteristic $p$ residue field. We suppose to have a sequence of $B$-algebra homomorphisms: $R \stackrel{\theta}{\rightarrow} S \stackrel{\mu}{\rightarrow} A$ of reduced local rings finite flat over $B$. We put $\lambda=\mu \circ \theta: R \rightarrow A$. We assume that $R, S, A$ are all Gorenstein rings over $B$. This means that

$$
\operatorname{Hom}_{B}(R, B) \cong R, \operatorname{Hom}_{B}(S, B) \cong S \text { and } \operatorname{Hom}_{B}(A, B) \cong A \text { as } R \text {-modules. }
$$

We write $B=\Lambda$. Since $R$ is reduced, the total quotient ring $Q(R)$ of $R$ is a product of fields, and we have $Q(R)=Q_{S} \oplus Q(S)$ for the complementary semi-simple algebra $Q_{S}$. Let $R_{S}$ be the projection of $R$ in $Q_{S}$. We have the following (unique) decomposition

(1) $\operatorname{Spec}(R)=\operatorname{Spec}\left(R_{S}\right) \cup \operatorname{Spec}(S)$, union of closed subschemes inducing $R \hookrightarrow\left(R_{S} \oplus S\right)$ with $\Lambda$-torsion module $C_{0}(\theta, S):=\left(R_{S} \oplus S\right) / R$.

Similarly, we have $Q(S)=Q_{A} \oplus Q(A)$ and $Q(R)=Q_{A}^{\prime} \oplus Q(A)$ as algebra direct sums. Write $S_{A}$ (resp. $R_{A}$ ) for the projected image of $S$ (resp. $R$ ) in $Q_{A}$ (resp. $Q_{A}^{\prime}$ ). Then we have 
(2) $\operatorname{Spec}(S)=\operatorname{Spec}\left(S_{A}\right) \cup \operatorname{Spec}(A)$, union of closed subschemes inducing $S \hookrightarrow\left(S_{A} \oplus A\right)$ with $\Lambda$-torsion module $C_{0}(\mu, A):=\left(S_{A} \oplus A\right) / S$.

(3) $\operatorname{Spec}(R)=\operatorname{Spec}\left(R_{A}\right) \cup \operatorname{Spec}(A)$, union of closed subschemes inducing $R \hookrightarrow\left(R_{A} \oplus A\right)$ with $\Lambda$-torsion module $C_{0}(\lambda, A):=\left(R_{A} \oplus A\right) / R$.

By [H88b, Lemma 6.3] (or [MFG, $§ 5.3 .3]$ ), we get the following isomorphisms of $R$-modules:

$$
C_{0}(\lambda ; A) \cong R_{A} \otimes_{R} A, C_{0}(\theta ; S) \cong R_{S} \otimes_{R} S \text { and } C_{0}(\mu ; A) \cong S_{A} \otimes_{S} A \text {. }
$$

Write $\pi_{S}: R \rightarrow R_{S}$ and $\pi: R \rightarrow S$ for the two projections and $(\cdot, \cdot)_{R}: R \times R \rightarrow B$ and $(\cdot, \cdot)_{S}: S \times S \rightarrow B$ for the pairing giving the self-duality (8.3). We recall [H86c, Lemma 1.6]:

Lemma 8.4. The $S$-ideal $\operatorname{Ker}\left(\pi_{S}: R \rightarrow R_{S}\right)$ is principal and $S$-free of rank 1 .

Proof. Let $\mathfrak{b}=\operatorname{Ker}(\theta: R \rightarrow S)$ and $\mathfrak{a}=\operatorname{Ker}\left(\pi_{S}: R \rightarrow R_{S}\right)$. By assumption, $R$ and $S$ are $B$-free of finite rank; so, $\mathfrak{b}$ is $B$-free, and by duality, we have an exact sequence $0 \rightarrow S^{*} \stackrel{\theta^{*}}{\rightarrow} R^{*} \rightarrow \mathfrak{b}^{*} \rightarrow 0$. Note that $\mathfrak{b}^{*}$ is naturally an $R_{S}$-module which is free of finite rank over $B$. Thus identifying $S=S^{*}$ and $R^{*}=R$ by (8.3), we have $\theta^{*}\left(S^{*}\right)=\{r \in R ; r \cdot \mathfrak{b}=0\}=(Q(S) \oplus 0) \cap R=\mathfrak{a}$; hence $\theta^{*}$ induces $S=S^{*} \cong \mathfrak{a}$.

Recall the following fact first proved in [H88b, Theorem 6.6]:

Lemma 8.5. We have the following exact sequence of R-modules:

$$
0 \rightarrow C_{0}(\mu ; A) \rightarrow C_{0}(\lambda ; A) \rightarrow C_{0}(\theta ; S) \otimes_{S} A \rightarrow 0 .
$$

Proof. Write $M^{*}=\operatorname{Hom}_{B}(M, B)$ as an $R$-module for a $R$-module $M$. Note that $\operatorname{Ker}(\theta)=R \cap\left(R_{S} \oplus 0\right) \subset R_{S} \oplus S, \operatorname{Ker}(\lambda)=R \cap\left(R_{A} \oplus 0\right) \subset R_{A} \oplus A$ and $\operatorname{Ker}(\mu)=S \cap\left(S_{A} \oplus 0\right) \subset S_{A} \oplus A$.

From an exact sequence $0 \rightarrow \operatorname{Ker}(\theta) \rightarrow R \rightarrow S \rightarrow 0$, we have the following commutative diagram with exact rows (for $\mathfrak{a}=\operatorname{Ker}\left(\pi_{S}: R \rightarrow R_{S}\right)$ ):

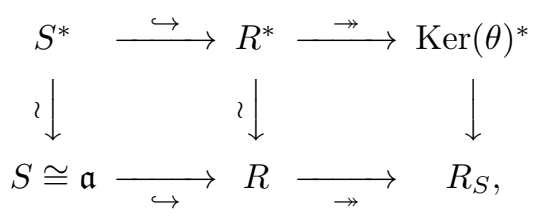

which shows $R_{S} \cong \operatorname{Ker}(\theta)^{*}=\left(\left(R_{S} \oplus 0\right) \cap R\right)^{*}$ as $R$-modules. Similarly, we get $\operatorname{Ker}(\lambda)^{*} \cong R_{A}$, $\operatorname{Ker}(\mu)^{*} \cong S_{A}$ We have a commutative diagram with exact rows:

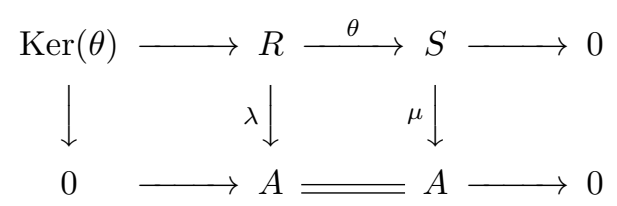

Applying the snake lemma, we get an exact sequence of $R$-modules:

$$
0 \rightarrow \operatorname{Ker}(\theta) \rightarrow \operatorname{Ker}(\lambda) \rightarrow \operatorname{Ker}(\mu) \rightarrow 0 .
$$

By $B$-freeness of $A$ and $S$, all the terms of the above exact sequence are $B$-free. Thus the above sequence is split as a sequence of $B$-modules, and we have the dual exact sequence:

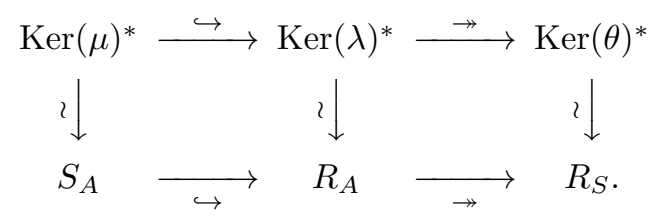

Tensoring with $A$ over $R$, from (8.4), we get an exact sequence:

$$
\operatorname{Tor}_{R}^{1}\left(R_{S}, A\right) \rightarrow C_{0}(\mu ; A) \rightarrow C_{0}(\lambda ; A) \rightarrow C_{0}(\theta ; S) \otimes_{R} A \rightarrow 0 .
$$


Thus we need to show the vanishing: $\operatorname{Tor}_{R}^{1}\left(R_{S}, A\right)=0$. To see this, we recall $\mathfrak{a} \cong S$. Thus the exact sequence $\mathfrak{a} \hookrightarrow R \rightarrow R_{S}$ can be rewritten as $S \hookrightarrow R \rightarrow R_{S}$. Tensoring with $A$ over $R$, we get an exact sequence

$$
0=\operatorname{Tor}_{R}^{1}(R, A) \rightarrow \operatorname{Tor}_{R}^{1}\left(R_{S}, A\right) \rightarrow S \otimes_{R} A \stackrel{\alpha}{\rightarrow} R \otimes_{R} A \rightarrow R_{S} \otimes_{R} A \rightarrow 0 .
$$

Since we have a commutative diagram:

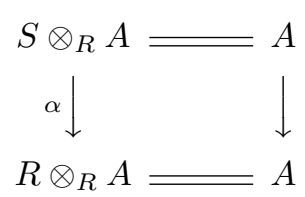

and $\operatorname{Coker}(\alpha)$ is a torsion $A$-module, $\alpha$ is a nontrivial $A$-linear map of the integral domain $A$ into itself; so, $\alpha$ is injective, and we conclude $\operatorname{Tor}_{R}^{1}\left(R_{S}, A\right)=0$ as desired.

By (8.4), the three congruence modules $C_{0}(\mu ; A), C_{0}(\lambda ; A), C_{0}(\theta ; S) \otimes_{R} A$ are residue rings of $R$; so, cyclic $A$-modules. Moreover, by Lemma 8.4, they are the ring $A$ modulo principal ideals. Write their generators as $A c_{\lambda}=A \cap R \subset\left(R_{A} \oplus A\right), A c_{\mu}=A \cap S \subset\left(S_{A} \oplus A\right)$ and $S c_{\theta}=S \cap R \subset\left(R_{S} \oplus S\right)$. Thus we have $C_{0}(\lambda ; A)=A / c_{\lambda} A, C_{0}(\mu ; A)=A / c_{\mu} A$ and $C_{0}(\theta ; S) \otimes_{S} A=A / \lambda\left(c_{\theta}\right) A$ for the image $\lambda\left(c_{\theta}\right) \in A$ of $c_{\theta} \in S$. By the above lemma, we conclude the following result:

Corollary 8.6. We have $\lambda\left(c_{\theta}\right) \cdot c_{\mu}=c_{\lambda}$ up to units in $A$; so, for the ideals $\mathfrak{c}_{\text {? }}$ generated by $c_{\text {? }}$, we have $\lambda\left(\mathfrak{c}_{\theta}\right) \cdot \mathfrak{c}_{\mu}=\mathfrak{c}_{\lambda}$.

Note here $\mathfrak{c}_{?}$ (resp. $\left.\lambda\left(\mathfrak{c}_{\theta}\right)\right)$ is the annihilator $\operatorname{Ann}_{A}\left(C_{0}(? ; A)\right)\left(\right.$ resp. $\operatorname{Ann}_{A}\left(C_{0}(? ; S) \otimes_{S} A\right)$ of $C_{0}(? ; A)$ in $A$, and $\mathfrak{c}_{\theta}$ is the annihilator $\operatorname{Ann}_{S}\left(C_{0}(\theta ; A)\right)$ of $C_{0}(\theta ; S)$ in $S$.

8.4. Local complete intersections. Let $A$ be a complete normal local domain (for example, a complete regular local rings like $A=W$ or $A=W[[T]]$ or $A=W\left[\left[T_{1}, \ldots, T_{r}\right]\right]$ (power series ring)). Any local $A$-algebra $R$ free of finite rank over $A$ has a presentation $R \cong A\left[\left[X_{1}, \ldots, X_{n}\right]\right] /\left(f_{1}, \ldots, f_{m}\right)$ for $f_{i} \in A\left[\left[X_{1}, \ldots, X_{n}\right]\right]$ with $m \geq n$. If $m=n$, then $R$ is called a local complete intersection over $A$. Note that if $B$ is a complete normal local domain which is finite flat over $A$, the extension $R \otimes_{A} B$ of an $A$-algebra $R$ which is local complete intersection over $A$ is local complete intersection over $B$. There is a theorem of Tate giving the identity of the Fitting ideals of the differential module and the congruence module for local complete intersection rings. To introduce this, let us explain the notion of pseudo-isomorphisms between torsion $A$-modules (see [BCM, VII.4.4] for a more detailed treatment). For two $A$-modules $M, N$ of finite type, a morphism $\phi: M \rightarrow N$ is called a pseudo isomorphism if the annihilator of $\operatorname{Ker}(\phi)$ and $\operatorname{Coker}(\phi)$ each has height at least 2 (i.e., the corresponding closed subscheme of $\operatorname{Spec}(A)$ has co-dimension at least 2). If $A=W$, a pseudo-isomorphism is an isomorphism, and if $A=W[[T]]$, it is an isogeny (having finite kernel and cokernel). The classification theorem of torsion $A$-modules $M$ of finite type tells us that we have a pseudo isomorphism $M \rightarrow \bigoplus_{i} A / \mathfrak{f}_{i}$ for finitely many reflexive ideal $0 \neq \mathfrak{f}_{i} \in A$. An ideal $\mathfrak{f}$ is reflexive if $\operatorname{Hom}_{A}\left(\operatorname{Hom}_{A}(\mathfrak{f}, A), A\right) \cong \mathfrak{f}$ canonically as $A$-modules (and equivalently $\mathfrak{f}=\bigcap_{\lambda \in A,(\lambda) \supset \mathfrak{f}}(\lambda)$; i.e., close to be principal). Then the characteristic ideal $\operatorname{Char}(M)$ of $M$ is defined by $\operatorname{Char}(M):=\prod_{i} \mathfrak{f}_{i} \subset A$. If $A$ is a unique factorization domain (for example, if $A$ is regular; a theorem of Auslander-Buchsbaum [CRT, Theorem 20.3]), any reflexive ideal is principal. If $A=W$, then $|W / \operatorname{Char}(M)|_{p}=|| M||_{p}$, and if further $A=\mathbb{Z}_{p}$, we have $\operatorname{Char}(M)=(|M|)$.

Theorem 8.7 (J. Tate). Assume that $R$ is a local complete intersection over a complete normal noetherian local domain $A$ with an algebra homomorphism $\lambda: R \rightarrow A$. If after tensoring the quotient field $Q$ or $A, R \otimes_{A} Q=\left(\operatorname{Im}(\lambda) \otimes_{A} Q\right) \oplus S$ as algebra direct sum for some $Q$-algebra $S$, then $C_{j}(\lambda ; A)$ is a torsion $A$-module of finite type, and we have

$$
\operatorname{Ann}_{A}\left(C_{0}(\lambda ; A)\right)=\operatorname{Char}\left(C_{0}(\lambda ; A)\right)=\operatorname{Char}\left(C_{1}(\lambda ; A)\right) .
$$

For the reader's convenience, we shall give a proof of this theorem in the following subsection. Actually we prove

$$
\operatorname{length}_{A}\left(C_{0}(\lambda ; A)\right)=\operatorname{length}_{A}\left(C_{1}(\lambda ; A)\right)
$$


assuming that $A$ is a discrete valuation ring (see Proposition 8.12). If $A$ is a normal noetherian domain, $\operatorname{Char}_{A}(M)=\prod_{P} P^{\text {length }_{A_{P}} M_{P}}$ for the localization $M_{P}$ at height 1-primes $P$ for a given $A$-torsion module $M$. Since $A_{P}$ is a discrete valuation ring if and only if $P$ has height 1 , this implies the above theorem.

8.5. Proof of Tate's theorem. We reproduce the proof from [MR70, Appendix] (which actually determines the Fitting ideal of $M$ more accurate than Char $(M))$. We prepare some preliminary results; so, we do not assume yet that $R$ is a local complete intersection over $A$. Let $A$ be a normal noetherian integral domain of characteristic 0 and $R$ be a reduced $A$-algebra free of finite rank $r$ over $A$. The algebra $R$ is called a Gorenstein algebra over $A$ if $\operatorname{Hom}_{A}(R, A) \cong R$ as $R$-modules. Since $R$ is free of rank $r$ over $A$, we choose a base $\left(x_{1}, \ldots, x_{r}\right)$ of $R$ over $A$. Then for each $y \in R$, we have $r \times r$-matrix $\rho(y)$ with entries in $A$ defined by $\left(y x_{1}, \ldots, y x_{r}\right)=\left(x_{1}, \ldots, x_{r}\right) \rho(y)$. Define $\operatorname{Tr}(y)=\operatorname{Tr}(\rho(y))$. Then $\operatorname{Tr}: R \rightarrow A$ is an $A$-linear map, well defined independently of the choice of the base. Suppose that $\operatorname{Tr}(x R)=0$. Then in particular, $\operatorname{Tr}\left(x^{n}\right)=0$ for all $n$. Therefore all eigenvalues of $\rho(x)$ are 0 , and hence $\rho(x)$ and $x$ is nilpotent. By the reducedness of $R, x=0$ and hence the pairing $(x, y)=\operatorname{Tr}(x y)$ on $R$ is non-degenerate.

Lemma 8.8. Let $A$ be a normal noetherian integral domain of characteristic 0 and $R$ be an $A-$ algebra. Suppose the following three conditions:

(1) $R$ is free of finite rank over $A$;

(2) $R$ is Gorenstein; i.e., we have $i: \operatorname{Hom}_{A}(R, A) \cong R$ as $R$-modules;

(3) $R$ is reduced.

Then for an A-algebra homomorphism $\lambda: R \rightarrow A$, we have

$$
C_{0}(\lambda ; A) \cong A / \lambda\left(i\left(\operatorname{Tr}_{R / A}\right)\right) A .
$$

In particular, length ${ }_{A} C_{0}(\lambda ; A)$ is equal to the valuation of $d=\lambda\left(i\left(\operatorname{Tr}_{R / A}\right)\right)$ if $A$ is a discrete valuation ring.

Proof. Let $\phi=i^{-1}(1)$. Then $\operatorname{Tr}_{R / A}=\delta \phi$. The element $\delta=\delta_{R / A}$ is called the different of $R / A$. Then the pairing $(x, y) \mapsto \operatorname{Tr}_{R / A}\left(\delta^{-1} x y\right) \in A$ is a perfect pairing over $A$, where $\delta^{-1} \in S=\operatorname{Frac}(R)$ and we have extended $\operatorname{Tr}_{R / A}$ to $S \rightarrow K=\operatorname{Frac}(A)$. Since $R$ is commutative, $(x y, z)=(y, x z)$. Decomposing $S=K \oplus X$, we have

$$
C_{0}(\lambda ; A)=\operatorname{Im}(\lambda) / \lambda(\mathfrak{a}) \cong A / R \cap(K \oplus 0) .
$$

Then it is easy to conclude that the pairing ( , ) induces a perfect $A$-duality between $R \cap(K \oplus 0)$ and $A \oplus 0$. Thus $R \cap(K \oplus 0)$ is generated by $\lambda(\delta)=\lambda\left(i\left(\operatorname{Tr}_{R / A}\right)\right)$.

Next we introduce two $A$-free resolutions of $R$, in order to compute $\delta_{R / A}$. We start slightly more generally. Let $X$ be an algebra. A sequence $f=\left(f_{1}, \ldots, f_{n}\right) \in X^{n}$ is called regular if $x \mapsto f_{j} x$ is injective on $X /\left(f_{1}, \ldots, f_{j-1}\right)$ for all $j=1, \ldots, n$. We now define a complex $K_{X}^{\bullet}(f)$ (called the Koszul complex) out of a regular sequence $f$ (see [CRT, Section 16]). Let $V=X^{n}$ with a standard base $e_{1}, \ldots, e_{n}$. Then we consider the exterior algebra

$$
\bigwedge \cdot V=\bigoplus_{j=0}^{n}\left(\wedge^{j} V\right)
$$

The graded piece $\wedge^{j} V$ has a base $e_{i_{1}, \ldots, i_{j}}=e_{i_{1}} \wedge e_{i_{2}} \wedge \cdots \wedge e_{i_{j}}$ indexed by sequences $\left(i_{1}, \ldots, i_{j}\right)$ satisfying $0<i_{1}<i_{2}<\cdots<i_{j} \leq n$. We agree to put $\bigwedge^{0} V=X$ and $\bigwedge^{j} V=0$ if $j>n$. Then we define $X$-linear differential $d: \bigwedge^{j} X \rightarrow \bigwedge^{j-1} X$ by

$$
d\left(e_{i_{1}} \wedge e_{i_{2}} \wedge \cdots \wedge e_{i_{j}}\right)=\sum_{r=1}^{j}(-1)^{r-1} f_{i_{r}} e_{i_{1}} \wedge \cdots \wedge e_{i_{r-1}} \wedge e_{i_{r+1}} \wedge \cdots \wedge e_{i_{j}} .
$$

In particular, $d\left(e_{j}\right)=f_{j}$ and hence,

$$
\bigwedge^{0} V / d\left(\bigwedge^{1} V\right)=X /(f)
$$


Thus, $\left(K_{X}^{\bullet}(f), d\right)$ is a complex and $X$-free resolusion of $X /\left(f_{1}, \ldots, f_{n}\right)$. We also have

$$
d_{n}\left(e_{1} \wedge e_{2} \wedge \cdots \wedge e_{n}\right)=\sum_{j=1}^{n}(-1)^{j-1} f_{j} e_{1} \wedge \cdots \wedge e_{j-1} \wedge e_{j+1} \wedge \cdots \wedge e_{n}
$$

Suppose now that $X$ is a $B$-algebra. Identifying $\wedge^{n-1} V$ with $V$ by

$$
e_{1} \wedge \cdots \wedge e_{j-1} \wedge e_{j+1} \wedge \cdots \wedge e_{n} \mapsto e_{j}
$$

and $\wedge^{n} V$ with $X$ by $e_{1} \wedge e_{2} \wedge \cdots \wedge e_{n} \mapsto 1$, we have

$$
\operatorname{Im}\left(d_{n}^{*}: \operatorname{Hom}_{B}\left(\bigwedge^{n-1} V, Y\right) \rightarrow \operatorname{Hom}_{B}\left(\bigwedge^{n} V, Y\right)\right) \cong(f) \operatorname{Hom}_{B}(X, Y),
$$

where $(f) \operatorname{Hom}_{B}(X, Y)=\sum_{j} f_{j} \operatorname{Hom}_{B}(X, Y)$, regarding $\operatorname{Hom}_{B}(X, Y)$ as an $X$-module by $y \phi(x)=$ $\phi(x y)$. This shows that if $X$ is an $B$-algebra free of finite rank over $B, K_{X}^{\bullet}(f)$ is a $B$-free resolution of $X /(f)$, and

$$
\operatorname{Ext}_{B}^{n}(X /(f), Y)=H^{n}\left(\operatorname{Hom}_{B}\left(K_{X}^{\bullet}(f), Y\right)\right) \cong \frac{\operatorname{Hom}_{B}(X, Y)}{(f) \operatorname{Hom}_{B}(X, Y)}
$$

for any $B$-module $Y$.

We now suppose that $R$ is a local complete intersection over $A$. Thus $R$ is free of finite rank over $A$ and $R \cong B /\left(f_{1}, \ldots, f_{n}\right)$ for $B=A\left[\left[T_{1}, \ldots, T_{n}\right]\right]$. Write $t_{j}$ for $T_{j} \bmod \left(f_{1}, \ldots, f_{n}\right)$ in $R$. Since $R$ is local, $t_{j}$ are contained in the maximal ideal $\mathfrak{m}_{R}$ of $R$. We consider $C=B \otimes_{A} R \cong R\left[\left[T_{1}, \ldots, T_{n}\right]\right]$. Then

$$
R=R\left[\left[T_{1}, \ldots, T_{n}\right]\right] /\left(T_{1}-t_{1}, \ldots, T_{n}-t_{n}\right),
$$

and $g=\left(T_{1}-t_{1}, \ldots, T_{n}-t_{n}\right)$ is a regular sequence in $C=R\left[\left[T_{1} \ldots, T_{n}\right]\right]$. Since $C$ is $B$-free of finite rank, the two complexes $K_{B}^{\bullet}(f) \rightarrow R$ and $K_{C}^{\bullet}(g) \rightarrow R$ are $B$-free resolutions of $R$.

We have a $\Lambda$-algebra homomorphism $\Phi: B \hookrightarrow C$ given by $\Phi(x)=x \otimes 1$. We extend $\Phi$ to $\Phi^{\bullet}$ : $K_{B}^{\bullet}(f) \rightarrow K_{C}^{\bullet}(g)$ in the following way. Write $f_{i}=\sum_{j=1}^{n} b_{i j} g_{j}$. Then we define $\Phi^{1}: K_{B}^{1}(f) \rightarrow K_{C}^{1}(g)$ by $\Phi^{1}\left(e_{i}\right)=\sum_{j=1}^{n} b_{i j} e_{j}$. Then $\Phi^{j}=\bigwedge^{j} \Phi^{1}$. One can check that this map $\Phi^{\bullet}$ is a morphism of complexes. In particular,

$$
\Phi_{n}\left(e_{1} \wedge \cdots \wedge e_{n}\right)=\operatorname{det}\left(b_{i j}\right) e_{1} \wedge \cdots \wedge e_{n} .
$$

Since $\Phi^{\bullet}$ is the lift of the identity map of $R$ to the $B$-projective resolutions $K_{B}^{\bullet}(f)$ and $K_{C}^{\bullet}(g)$, it induces an isomorphism of extension groups computed by $K_{C}^{\bullet}(g)$ and $K_{B}^{\bullet}(f)$ :

$$
\Phi^{*}: H^{\bullet}\left(\operatorname{Hom}_{B}\left(K_{C}^{\bullet}(g), B\right)\right) \cong \operatorname{Ext}_{B}^{j}(R, B) \cong H^{\bullet}\left(\operatorname{Hom}_{B}\left(K_{B}^{\bullet}(f), B\right)\right) .
$$

In particular, identifying $\wedge^{n} B^{n}=B$, we have from (8.6) that

$$
H^{n}\left(\operatorname{Hom}_{B}\left(K_{B}^{\bullet}(f), B\right)\right)=\operatorname{Hom}_{B}(B, B) /(f) \operatorname{Hom}_{B}(B, B)=B /(f)=R
$$

and similarly

$$
H^{n}\left(\operatorname{Hom}_{B}\left(K_{C}^{\bullet}(g), B\right)\right)=\frac{\operatorname{Hom}_{B}(C, B)}{(g) \operatorname{Hom}_{B}(C, B)} .
$$

The isomorphism between $R$ and $\frac{\operatorname{Hom}_{B}(C, B)}{(g) \operatorname{Hom}_{B}(C, B)}$ is induced by $\Phi_{n}$ which is a multiplication by $d=$ $\operatorname{det}\left(b_{i j}\right)$ (see (8.7)). Thus we have

Lemma 8.9. Suppose that $R$ is a local complete intersection over $A$. Let $\pi: B=A\left[\left[T_{1}, \ldots, T_{n}\right]\right] \rightarrow$ $R$ be the projection as above. We have an isomorphism:

$$
h: \frac{\operatorname{Hom}_{B}(C, B)}{\left(T_{1}-t_{1}, \ldots, T_{n}-t_{n}\right) \operatorname{Hom}_{B}(C, B)} \cong R
$$

given by $h(\phi)=\pi(\phi(d))$ for $d=\operatorname{det}\left(b_{i j}\right) \in C$.

We have a base change map:

$$
\iota: \operatorname{Hom}_{A}(R, A) \longrightarrow \operatorname{Hom}_{B}(C, B)=\operatorname{Hom}_{B}\left(B \otimes_{A} R, B \otimes_{A} A\right),
$$

taking $\phi$ to id $\otimes \phi$. Identifying $C$ and $B$ with power series rings, $\iota(\phi)$ is just applying the original $\phi$ to coefficients of power series in $R\left[\left[T_{1}, \ldots, T_{n}\right]\right]$. We define $I=h \circ \iota: \operatorname{Hom}_{A}(R, A) \rightarrow R$. 
Lemma 8.10. Suppose that $R$ is a local complete intersection over $A$. Then the above map $I$ is an $R$-linear isomorphism, satisfying $I(\phi)=\pi(\iota(\phi(d))$. Thus the ring $R$ is Gorenstein.

Proof. We first check that $I$ is an $R$-linear map. Since $I(\phi)=\pi(\iota(\phi(d))$, we compute $I(\phi \circ b))$ and $r I(\phi)$ for $b \in B$ and $r=\pi(b)$. By definition, we see

$$
I(\pi(b x))=\pi(\iota(\phi(r \otimes 1) d)) \text { and } r I(\phi)=\pi(b \iota(\phi(d)) .
$$

Thus we need to check $\pi(\iota(\phi)((r \otimes 1-1 \otimes b) d))=0$. This follows from:

$$
r \otimes 1-1 \otimes b \in(g) \text { and } \operatorname{det}\left(b_{i j}\right) g_{i}=\sum_{i} b_{i j}^{\prime} f_{i},
$$

where $b_{i j}^{\prime}$ are the $(i, j)$-cofactors of the matrix $\left(b_{i j}\right)$. Thus $I$ is $R$-linear. Since $\iota \bmod \mathfrak{m}_{B}$ for the maximal ideal $\mathfrak{m}_{B}$ of $B$ is a surjective isomorphism from

$$
\operatorname{Hom}_{A}\left(\left(A / \mathfrak{m}_{A}\right)^{r}, A / \mathfrak{m}_{A}\right)=\operatorname{Hom}_{A}(R, A) \otimes_{A} A / \mathfrak{m}_{A}
$$

onto

$$
\operatorname{Hom}_{B}\left(\left(B / \mathfrak{m}_{B}\right)^{r}, B / \mathfrak{m}_{B}\right)=\operatorname{Hom}_{B}(C, B) \otimes_{B} B / \mathfrak{m}_{B},
$$

the map $\iota$ is non-trivial modulo $\mathfrak{m}_{C}$. Thus $I \bmod \mathfrak{m}_{R}$ is non-trivial. Since $h$ is an isomorphism, $\operatorname{Hom}_{B}(C, B) \otimes_{C} C / \mathfrak{m}_{C}$ is 1-dimensional, and hence $I \bmod \mathfrak{m}_{R}$ is surjective. By Nakayama's lemma, $I$ itself is surjective. Since the target and the source of $I$ are $A$-free of equal rank, the surjectivity of $I$ tells us its injectivity. This finishes the proof.

Corollary 8.11. Suppose that $R$ is a local complete intersection over $A$. We have $I\left(\operatorname{Tr}_{R / A}\right)=\pi(d)$ for $d=\operatorname{det}\left(b_{i j}\right)$, and hence the different $\delta_{R / A}$ is equal to $\pi(d)$.

Proof. The last assertion follows from the first by $I(\phi)=\pi(\iota(\phi(d))$. To show the first, we choose dual basis $x_{1}, \ldots, x_{r}$ of $R / A$ and $\phi_{1}, \ldots, \phi_{r}$ of $\operatorname{Hom}_{A}(R, A)$. Thus for $x \in R$, writing $x x_{i}=\sum_{i} a_{i j} x_{j}$, we have $\operatorname{Tr}(x)=\sum_{i} a_{i i}=\sum_{i} \phi_{i}\left(x x_{i}\right)=\sum_{i} x_{i} \phi_{i}(x)$. Thus $\operatorname{Tr}=\sum_{i} x_{i} \phi_{i}$.

Since $x_{i}$ is also a base of $C$ over $B$, we can write $d=\sum_{j} b_{j} x_{i}$ with $\iota\left(\phi_{i}\right)(d)=b_{i}$. Then we have

$$
I\left(\operatorname{Tr}_{R / A}\right)=\sum_{i} x_{i} I\left(\phi_{i}\right)=\sum_{i} x_{i} \pi\left(\iota\left(\phi_{i}\right)(d)\right)=\sum_{i} x_{i} \pi\left(b_{i}\right)=\pi\left(\sum_{i} b_{i} x_{i}\right)=\pi(d) .
$$

This shows the desired assertion.

We now finish the proof of (8.5):

Proposition 8.12. Let $A$ be a discrete valuation ring, and let $R$ be a reduced local complete intersection over $A$. Then for an A-algebra homomorphism $R \rightarrow A$, we have

$$
\text { length }_{A} C_{0}(\lambda, A)=\operatorname{length}_{A} C_{1}(\lambda, A) .
$$

Proof. Let $X$ be a torsion $A$-module, and suppose that we have an exact sequence:

$$
A^{r} \stackrel{L}{\rightarrow} A^{r} \rightarrow X \rightarrow 0
$$

of $A$-modules. Then we claim length $A=\operatorname{length}_{A} A / \operatorname{det}(L) A$. By elementary divisor theory applied to $L$, we may assume that $L$ is a diagonal matrix with diagonal entry $d_{1}, \ldots, d_{r}$. Then the assertion is clear, because $X=\bigoplus_{j} A / d_{j} A$ and length $A / d A$ is equal to the valuation of $d$.

Since $R$ is reduced, $\Omega_{R / A}$ is a torsion $R$-module, and hence $\Omega_{R / A} \otimes_{R} A=C_{1}(\lambda ; A)$ is a torsion $A$-module. Since $R$ is a local complete intersection over $A$, we can write

$$
R \cong A\left[\left[T_{1}, \ldots, T_{r}\right]\right] /\left(f_{1}, \ldots, f_{r}\right) .
$$

Then by Corollary 8.2 (ii), we have the following exact sequence for $J=\left(f_{1}, \ldots, f_{r}\right)$ :

$$
J / J^{2} \otimes_{A\left[\left[T_{1}, \ldots, T_{r}\right]\right]} A \longrightarrow \Omega_{A\left[\left[T_{1}, \ldots, T_{r}\right]\right] / A} \otimes_{A\left[\left[T_{1}, \ldots, T_{r}\right]\right]} A \longrightarrow \Omega_{R / A} \otimes_{R} A \rightarrow 0 .
$$

This gives rise to the following exact sequence:

$$
\bigoplus_{j} A d f_{j} \stackrel{L}{\rightarrow} \bigoplus_{j} A d T_{j} \longrightarrow C_{1}(\lambda ; A) \rightarrow 0,
$$


where $d f_{j}=f_{j} \bmod J^{2}$. Since $C_{1}(\lambda ; A)$ is a torsion $A$-module, we see that length ${ }_{A}(A / \operatorname{det}(L) A)=$ length $_{A} C_{1}(\lambda ; A)$. Since $g=\left(T_{1}-t_{1}, \ldots, T_{n}-t_{n}\right)$, we see easily that $\operatorname{det}(L)=\pi(\lambda(d))$. This combined with Corollary 8.11 and Lemma 8.8 shows the desired assertion.

8.6. A more general setting. Let $\boldsymbol{\Lambda}_{j}$ be the power series ring $W\left[\left[T_{1}, \ldots, T_{j}\right]\right]$. We consider the following commutative diagram of local profinite $W$-algebras sharing the same residue field $\mathbb{F}$ with $W$ :

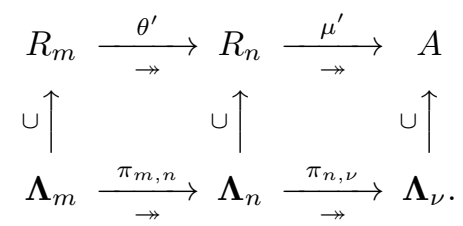

We put $\lambda^{\prime}:=\mu^{\prime} \circ \theta^{\prime}$. Consider the following conditions:

(A0) $A$ is an integral domain.

(A1) $\pi_{j, k}$ indices the identity $\boldsymbol{\Lambda}_{j} \otimes_{\boldsymbol{\Lambda}_{j}, \pi_{j, k}} \boldsymbol{\Lambda}_{k}=\boldsymbol{\Lambda}_{k}$ for $(j, k)=(m, n)$ and $(j, k)=(n, \nu)$; so, $m \geq n \geq \nu$.

(A2) $R_{j}$ is free of finite rank over $\boldsymbol{\Lambda}_{j}$ for $j=m, n$, and $A$ is a torsion-free $\boldsymbol{\Lambda}_{\nu}$-modules of finite type.

(A3) $\operatorname{Hom}_{\boldsymbol{\Lambda}_{j}}\left(R_{j}, \boldsymbol{\Lambda}_{j}\right) \cong R_{j}$ as $R_{j}$-modules for $j=m, n$.

(A4) $R_{j} \otimes_{\boldsymbol{\Lambda}_{j}} \boldsymbol{\Lambda}_{\nu}$ is a reduced algebra for $j=m, n$.

(A5) $R_{j}$ is a local complete intersection over $\boldsymbol{\Lambda}_{j}$ for $j=m, n$.

Note that (A5) implies (A4) (e.g., [CRT, Theorem 21.3]).

Lemma 8.13. Suppose (A0-3). Let $\widetilde{A}$ be the normalization of $A$ and put $\widetilde{R}_{j}=R_{j} \otimes_{\boldsymbol{\Lambda}_{j}} \widetilde{A}$ for $j=m, n$.

(1) Suppose that $\nu=1$. Then $\widetilde{R}_{j}$ is free of finite rank over $\widetilde{A}$ and satisfies $\operatorname{Hom}_{\widetilde{A}}\left(\widetilde{R}_{j}, \widetilde{A}\right) \cong \widetilde{R}_{j}$ as $\widetilde{R}_{j}$-modules;

(2) Suppose $\nu>1$. Then for each height 1 prime $P$ of $\widetilde{A}, \widetilde{R}_{j, P}=\widetilde{R}_{j} \otimes_{\widetilde{A}} \widetilde{A}_{P}$ for the localization $\widetilde{A}_{P}$ of $\widetilde{A}$ at $P$ is free of finite rank over $\widetilde{A}_{P}$ and satisfies $\operatorname{Hom}_{\widetilde{A}_{P}}\left(\widetilde{R}_{j, P}, \widetilde{A}_{P}\right) \cong \widetilde{R}_{j, P}$ as $\widetilde{R}_{j, P}$-modules for $j=m, n$.

Proof. We only prove the assertion (1), since the assertion (2) is easier to prove after localization at $P$ as $\widetilde{A}_{P}$ is a discrete valuation ring. If $\nu=1, \widetilde{A}$ is reflexive and hence flat over $\boldsymbol{\Lambda}_{1}$. Thus we get

$$
\operatorname{Hom}_{\boldsymbol{\Lambda}_{j}}\left(R_{j}, \boldsymbol{\Lambda}_{j}\right) \otimes_{\boldsymbol{\Lambda}_{j}} \widetilde{A} \cong \operatorname{Hom}_{\widetilde{A}}\left(\widetilde{R}_{j}, \widetilde{A}\right)
$$

from [BAL, II.5.4]. Since $\operatorname{Hom}_{\boldsymbol{\Lambda}_{j}}\left(R_{j}, \boldsymbol{\Lambda}_{j}\right) \cong R_{j}$, we get from the above identity

$$
\widetilde{R}_{j}=R_{j} \otimes_{\boldsymbol{\Lambda}_{j}} \widetilde{A} \cong \operatorname{Hom}_{\boldsymbol{\Lambda}_{j}}\left(R_{j}, \boldsymbol{\Lambda}_{j}\right) \otimes_{\boldsymbol{\Lambda}_{j}} \widetilde{A} \cong \operatorname{Hom}_{\widetilde{A}}\left(\widetilde{R}_{j}, \widetilde{A}\right)
$$

as $\widetilde{R}_{j}$-modules.

By Lemma 8.13, under (A0-4), the sequences $\widetilde{R}_{m} \stackrel{\theta}{\rightarrow} \widetilde{R}_{n} \stackrel{\mu}{\rightarrow} \widetilde{A}$ if $\nu=1$ and $\widetilde{R}_{m, P} \stackrel{\theta}{\rightarrow} \widetilde{R}_{n, P} \stackrel{\mu}{\rightarrow} \widetilde{A}_{P}$ if $\nu=1$ for $\theta=\theta^{\prime} \otimes 1$ and $\mu=\mu^{\prime} \otimes 1$ satisfies the requirement of $R \stackrel{\theta}{\rightarrow} S \stackrel{\mu}{\rightarrow} A$ for the transfer property of congruence modules in 8.3. Thus we get for the annihilators $\mathfrak{c}_{\text {? }}:=\operatorname{Ann}_{\widetilde{A}}\left(C_{0}(? ; \widetilde{A})\right)$ with $?=\lambda, \mu$ and $\mathfrak{c}_{\theta}:=\operatorname{Ann}_{\widetilde{R}_{n}}\left(C_{0}\left(\theta ; \widetilde{R}_{n}\right)\right)$ the following transfer formula:

Proposition 8.14. Assume (A0-4).

(1) If $\nu=1$, the ideals $\mathfrak{c}_{?}(?=\lambda, \mu, \theta)$ are all principal, and satisfies $\lambda\left(\mathfrak{c}_{\theta}\right) \cdot \mathfrak{c}_{\mu}=\mathfrak{c}_{\lambda}$.

(2) If $\nu>1$, writing $\widetilde{M}$ for the reflexive closure of a torsion-free $\boldsymbol{\Lambda}_{\nu}$-module $M$ of finite type, we have the following identity $\lambda \widetilde{\left(\mathfrak{c}_{\theta}\right) \cdot \mathfrak{c}_{\mu}}=\widetilde{\mathfrak{c}_{\lambda}}$.

Proof. By Corollary 8.6, we get the assertion (1) and also the localized identity: $\lambda\left(\mathfrak{c}_{\theta}\right)_{P} \cdot \mathfrak{c}_{\mu, P}=\mathfrak{c}_{\lambda, P}$ for each height 1 prime $P$ in the setting of (2), since $\lambda\left(\mathfrak{c}_{\theta, P}\right)=\lambda\left(\mathfrak{c}_{\theta}\right)_{P}$ and $\mathfrak{c}_{?, P}$ is the annihilator of the corresponding $P$-localized congruence module by the definition of the congruence module. Since $\widetilde{M}=\bigcap_{P} M_{P}$ inside $M \otimes_{\widetilde{A}} \operatorname{Frac}(\widetilde{A})$, we get the assertion (2). 
Now suppose (A5); so, $R_{j} \cong \boldsymbol{\Lambda}_{j}\left[\left[X_{1}, \ldots, X_{k}\right]\right] /\left(f_{1}, \ldots, f_{k}\right)$ for a regular sequence $\left(f_{1}, \ldots, f_{k}\right)$ in $\mathfrak{m}_{\boldsymbol{\Lambda}_{j}\left[\left[X_{1}, \ldots, X_{k}\right]\right]}$. Tensoring $\widetilde{A}$ over $\boldsymbol{\Lambda}_{j}$ with the exact sequence:

$$
0 \rightarrow\left(f_{1}, \ldots, f_{k}\right) \rightarrow \boldsymbol{\Lambda}_{j}\left[\left[X_{1}, \ldots, X_{k}\right]\right] \rightarrow R_{j} \rightarrow 0,
$$

we get a sequence,

$$
0 \rightarrow\left(f_{1}, \ldots, f_{k}\right) \rightarrow \widetilde{A}\left[\left[X_{1}, \ldots, X_{k}\right]\right] \rightarrow \widetilde{R}_{j} \rightarrow 0,
$$

which is exact. Since $R_{j}$ is $\boldsymbol{\Lambda}_{j}$-free of finite rank, the first sequence of $\boldsymbol{\Lambda}_{j}$-modules is split exact; so, the exactness is kept after tensoring $\widetilde{A}$. Thus (A5) implies that

$\left(\mathrm{A}^{\prime} 5\right) \widetilde{R}_{j}$ is a local complete intersection over $\widetilde{A}$ for $j=m, n$.

Thus we may apply Tate's formula Theorem 8.7 to our setting $\widetilde{R}_{m, P} \rightarrow \widetilde{R}_{n, P} \rightarrow \widetilde{A}_{P}$ for each height 1 primes and get the following fact:

Theorem 8.15. Assume (A0-5). Then we have

$$
\widetilde{\lambda\left(\mathfrak{c}_{\theta}\right)} \cdot \operatorname{Char}\left(C_{1}(\mu, \widetilde{A})\right)=\operatorname{Char}\left(C_{1}(\lambda, \widetilde{A})\right) .
$$

\section{REFERENCES}

[AC89] J. Arthur, L. Clozel, Simple algebras, Base Change and the Advanced Theory of the Trace Formula, Ann. of Math. Studies, Princeton U. Press, 1989

[BAL] N. Bourbaki, Algébre, Chapitre 2, Hermann, Paris, 1962.

[BCM] N. Bourbaki, Algèbre Commutative, Hermann, Paris, 1961-1998.

[CRT] H. Matsumura, Commutative Ring Theory, Cambridge studies in advanced mathematics 8, Cambridge Univ. Press, 1986

[CHT08] L. Clozel, M. Harris, R. Taylor, Automorphy for some $\ell$-adic lifts of automorphic modulo $\ell$ Galois representations, Publ. Math. Inst. Hautes Études Sci. (2008), no. 108, 1-181, With Appendix A, summarizing unpublished work of Russ Mann, and Appendix B by Marie-France Vignéras. MR MR2470687

[Clo91] L. Clozel, Représentations galoisiennes associées aux représentations automorphes autoduales de $G L(n)$, Publications Mathématiques de l'IHÉS, 73 (1991), p. 97-145

[CT14] L. Clozel, J. Thorne, Level-raising and symmetric power functoriality, I. Compositio Mathematica, Vol. 150 (2014), No. 5, pp 729-748

[CT15] L. Clozel, J. Thorne, Level-raising and symmetric power functoriality, II. Annals of Mathematics, Vol. 181 (2015), No. 1, pp. 303-359

[Con16a] A. Conti, Grande image de Galois pour les familles p-adiques de formes automorphes de pente positive, thèse de l'Université Paris 13, defended July 13, 2016

[Con16b] A. Conti, Galois level and congruence ideal for $\mathrm{GSp}_{4}$, preprint, $65 \mathrm{pp}$

[DDT94] H. Darmon, F. Diamond, R. Taylor, Fermat's Last Theorem, 1994

[Dia97] F. Diamond, The Taylor-Wiles construction and multiplicity one, Invent. Math., 128, (1997), 379-391.

[GeTi05] A. Genestier, J. Tilouine, Systèmes de Taylor-Wiles pour GSp4, in Formes automorphes II. Le cas du groupe GSp(4), Astérisque 302, Soc. Math. France, Paris, 2005, pp. 177-290

[GT11] W.-T. Gan, S. Takeda, The local Langlands conjecture for GSp(4), Ann. of Math., vol. 173 (2011), pp. 1841-1882

[Ge10] D. Geraghty, Modularity lifting theorems for ordinary Galois representations, Harvard Dissertation 2010

[Ge16] D. Geraghty, Notes on modularity lifting in the ordinary case, in p-adic aspects of modular forms, Proc. IISER Pune conference, eds B. Balasubramaniam, H. Hida, A. Raghuram, J. Tilouine, World Scientific Publ. 2016

[HT01] M. Harris, R. Taylor, The geometry and cohomology of some simple Shimura varieties, Annals of Mathematics Studies, vol. 151, Princeton University Press, Princeton, NJ, 2001, With an appendix by Vladimir G. Berkovich

[HJ] R. Harron, A. Jorza, On symmetric power $\mathcal{L}$-invariants of Iwahori level Hilbert modular forms, preprint

[H86c] H. Hida, Hecke algebras for $G L_{1}$ and $G L_{2}$, Sém. de Théorie des Nombres, Paris 1984-85, Progress in Math. 63 (1986), 131-163

[H88b] H. Hida, Modules of congruence of Hecke algebras and $L$-functions associated with cusp forms, Amer. J. Math. 110 (1988), 323-382

[H02] H. Hida, Control theorems of coherent sheaves on Shimura varieties of PEL type, J. Inst. Math. Jussieu, 1, 2002, pp.1-76

[GME] H. Hida, Geometric Modular Forms and Elliptic Curves, second edition, World Scientific, Singapore, 2011

[MFG] H. Hida, Modular Forms and Galois Cohomology, Cambridge Studies in Advanced Mathematics 69, Cambridge University Press, Cambridge, England, 2000 (a list of errata posted at www.math.ucla.edu/ ${ }^{\sim h i d a) . ~}$

[PAF] H. Hida, $p$-adic Automorphic Forms, Springer Monographs in Mathematics, Springer Verlag, 2004

[H88b] H. Hida, Modules of congruence of Hecke algebras and Lfunctions associated with cusp forms, Amer. J. Math. 110 (1988), 323382 
[H16] H. Hida, Arithmetic of adjoint L-values, in p-adic aspects of modular forms, Proc. IISER Pune Conference, eds B. Balasubramaniam, H. Hida, A. Raghuram, J. Tilouine, World Scientific Publ. 2016

[HT15] H. Hida, J. Tilouine, Big image of Galois representations and congruence ideals, in Arithmetic Geometry, pp. 217-254, Proc. Workshop on Serre's Conjecture, Hausdorff Inst. Math., Bonn, eds. L. Dieulefait, D.R. Heath-Brown, G. Faltings, Y.I. Manin, B. Z. Moroz, J.-P. Wintenberger, Cambridge University Press (2015).

[Kim03] H. H. Kim, Functoriality for the exterior square of $G L_{4}$ and the symmetric fourth of $G L_{2}$, J. Amer. Math. Soc. 16 (2003), no. 1, 139183. With appendix 1 by Dinakar Ramakrishnan and appendix 2 by Kim and Peter Sarnak

[KS02a] H. H. Kim and F. Shahidi, Cuspidality of symmetric powers with applications, Duke Math. J. 112 (2002), pp.177-197

[KS02b] H. H. Kim and F. Shahidi, Functorial products for $G L_{2} \times G L_{3}$ and the symmetric cube for $G L_{2}$, Ann. of Math. (2) 155 (2002), no. 3, 837-893. With an appendix by Colin J. Bushnell and Guy Henniart

[Lab09] J.-P. Labesse, Changement de base CM et séries discrètes, in Book ed. M. Harris 2009.

[Lau05] G. Laumon, Fonctions zêta des variétés de Siegel de dimension trois, in Formes Automorphes (II), le cas du groupe GSp(4), Astérisque 302, SMF, 2005

[Mok14] C. P. Mok, Galois representations attached to automorphic forms on GL 2 over a CM field, Compos. Math. 150, (2014), pp 523-567

[MR70] B. Mazur and L. Robert, Local Euler characteristic, Invent. Math. 9 (1970), 201-234, with an Appendix by J. Tate

[Pi12b] V. Pilloni, Modularité, formes de Siegel et surfaces abéliennes, J. reine angew. Math. 666, 2012, 35-82

[PR94] V. Platonov, A. Rapinchuk, Algebraic Groups and Number Theory, Acad. Press 1994

[PT02] P. Polo, J. Tilouine, Bernstein-Gelfand-Gelfand complexes and cohomology of nilpotent groups over $\mathbb{Z}_{p}$, Astérisque 282, SMF, 2002

[Ri76] K. Ribet, A modular construction of unramified $p$-extensions of $\mathbb{Q}\left(\mu_{p}\right)$, Inv. Math. 34, 151-162, 1976

[RS07] D. Ramakrishnan, F. Shahidi, Siegel modular forms of genus two attached to elliptic curves, Math. Res. Lett. 14 (2007), fasc. 2, pp.315-332

[RoS07] Roberts B., Schmidt R., Local Newforms on GSp(4), Springer LNM 1918, Springer Verlag 2007

[TU99] J. Tilouine, E. Urban, Several variable p-adic families of Siegel-Hilbert cusp eigensystems and their Galois representations, Ann. Sci. E.N.S., 4 série, t. 32, p. 499-574, 1999

[Ti02] J. Tilouine, Deformations of Galois representations and Hecke algebras, Mehta Institute, AMS 2002

[Ti06] J. Tilouine, Nearly ordinary rank four Galois representations and p-adic Siegel modular forms, Compos. Math.142 (2006), 1122-1156

[Vi84] M.-F. Vignéras, On the global correspondence between $\mathrm{GL}(n)$ and a division algebra, Institute for Advanced Studies, Princeton 1984

[We05] R. Weissauer, Four-dimensional Galois representations, in Formes Automorphes (II), le cas du groupe GSp(4), pp., Astérisque 302, SMF, 2005

[Y79] H. Yoshida, Weil's representations and Siegel's modular forms. Lectures on harmonic analysis on Lie groups and related topics (Strasbourg, 1979), pp. 319-341, Lectures in Math., 14, Kinokuniya Book Store, Tokyo, 1982.

Department of Mathematics, UCla, Los Angeles, CA 90095-1555, U.S.A., Department of Mathematics, U. Paris 13, Villetaneuse 93430, France.

E-mail address: hida@math.ucla.edu, tilouine@math.univ-paris13.fr 